\title{
LA FAMILIA ASTERACEAE EN EL ESTADO DE ZACATECAS (MÉXICO)
}

\author{
José de Jesús BALleza \\ Universidad Autónoma de Zacatecas \\ Facultad de Agronomía \\ km 15.5, carretera Zacatecas-Guadalajara \\ 98171 Zacatecas, Zacatecas \\ Y \\ José LUIS VILLASEÑOR \\ Instituto de Biología, UNAM \\ Departamento de Botánica \\ Apartado postal 70-367 \\ 04510 México, D.F.
}

\section{RESUMEN}

Con base en un intenso trabajo de campo, en la revisión crítica de la colección de Asteraceae de tres de los principales herbarios de México y en la consulta de la literatura florísticotaxonómica disponible, se presenta una lista actualizada de las especies de Asteraceae que crecen en el estado de Zacatecas. El catálogo está integrado por 141 géneros, 456 especies y 119 taxa infraespecíficos, repartidos en 14 tribus. Las tribus más diversas en el estado son Heliantheae (50 géneros y 149 especies), Astereae (22 y 68) y Eupatorieae (17 y 96). Por otra parte, los géneros con mayor número de especies son Stevia (27), Ageratina (23), Brickellia (21), Viguiera (15), Verbesina (14), Pseudognaphalium (13) y Acourtia (12). De las especies encontradas, 54\% son endémicas de México y cuatro especies y una variedad sólo se conocen del estado. El mayor número de endemismos se comparte con Durango y Jalisco. Se discuten los patrones de distribución geográfica de las especies, se confronta la riqueza observada en Zacatecas con la conocida de estados vecinos y se compara la diversidad de Asteraceae con otras familias presentes en el área de estudio. Las Asteraceae constituyen el grupo más numeroso de todas las familias de fanerógamas en Zacatecas; esta riqueza florística es en gran parte resultado de la convergencia en el estado de las provincias fisiográficas Sierra Madre Occidental, Sierra Madre Oriental, Mesa del Centro y Eje Neovolcánico y a la conjunción en su territorio de los reinos biogeográficos Holártico y Neotropical.

Palabras clave: Asteraceae, México, Zacatecas.

\begin{abstract}
Based on an intense fieldwork, on the critical review of the specimens of Asteraceae held in three important Mexican herbaria, as well as the study of specialized literature, a checklist of the Asteraceae of the state of Zacatecas is provided. The checklist includes 141 genera, 456 species,
\end{abstract}


Acta Botanica Mexicana (2002), 59: 5-69

and 119 infraspecific taxa, distributed in 14 tribes. The tribes with the largest number of taxa are Heliantheae (50 genera and 149 species), Astereae (22 and 68), and Eupatorieae (17 and 96). The most diverse genera are Stevia (27 species), Ageratina (23), Brickellia (21), Viguiera (15), Verbesina (14), Pseudognaphalium (13) and Acourtia (12). Fifty four percent of the species occurring in Zacatecas are endemic to Mexico, four of them plus one variety are restricted to the political boundaries of the state. The largest number of endemisms are shared with Durango and Jalisco, two neighbor states. In Zacatecas the Asteraceae constitute the most diversified family of flowering plants. Its richness is largely the result of the confluence in the state of four physiographic provinces (Sierra Madre Occidental, Sierra Madre Oriental, Central Plateau and Transvolcanic Belt), as well as of the confluence of the Holarctic and Neotropical Floristic Kingdoms.

Key words: Asteraceae, Mexico, Zacatecas.

\section{INTRODUCCIÓN}

La familia Asteraceae ocupa un lugar preponderante en la flora de México, tanto a nivel de géneros como de especies y contribuye substancialmente a la enorme riqueza florística de nuestro país (Villaseñor, 1993). Actualmente se registran para el territorio de la República 3,005 especies nativas de Asteraceae, distribuidas en 392 géneros (Villaseñor, datos no publicados). Alrededor de $66 \%$ de las especies restringen su área de distribución al territorio nacional. Tales cantidades aumentan continuamente debido a la exploración de áreas que hasta ahora habían sido poco estudiadas (compárese por ejemplo las cifras aquí mencionadas con las citadas por Turner y Nesom, 1998).

De la flora nacional, la familia Asteraceae es una de las más estudiadas; sin embargo, aún se tiene insuficiente conocimiento de ella a nivel regional o estatal. Existen entidades federativas que carecen todavía de inventarios florísticos y en consecuencia de un recuento actualizado del número de especies de Asteraceae que contienen. Entre ellas se cuenta Zacatecas, la cual hasta la fecha no ha sido objeto de estudio florístico detallado. En consecuencia, los objetivos de este trabajo consisten en presentar un catálogo actualizado de los componentes de esta familia que forman parte de la flora de Zacatecas, determinar la magnitud del elemento endémico, así como comparar su riqueza con la que se registra en los estados vecinos y con la de otras familias de fanerógamas presentes en el área de estudio.

\section{ÁREA DE ESTUDIO}

Zacatecas está situado en la porción centro-norte de la República Mexicana, entre los paralelos $21^{\circ} 04^{\prime}$ y $25^{\circ} 09^{\prime}$ de latitud norte y los meridianos $100^{\circ} 40^{\prime}$ y $104^{\circ} 19^{\prime}$ de longitud oeste. Posee una superficie de $74,668 \mathrm{~km}^{2}$, que corresponde a $3.7 \%$ de la superficie total del país. Se divide políticamente en 56 municipios (Fig. 1). Limita al norte con los estados de Durango y Coahuila, al sur con Aguascalientes y Jalisco, al este con San Luis Potosí y al oeste con Durango(Anónimo, 1981).

En Zacatecas convergen las provincias florísticas Sierra Madre Occidental, Sierra Madre Oriental, Altiplanicie y Costa Pacífica (Rzedowski, 1978; Fig. 2). Las dos primeras se agrupan en la Región Mesoamericana de Montaña y muestran influencia de los Reinos 


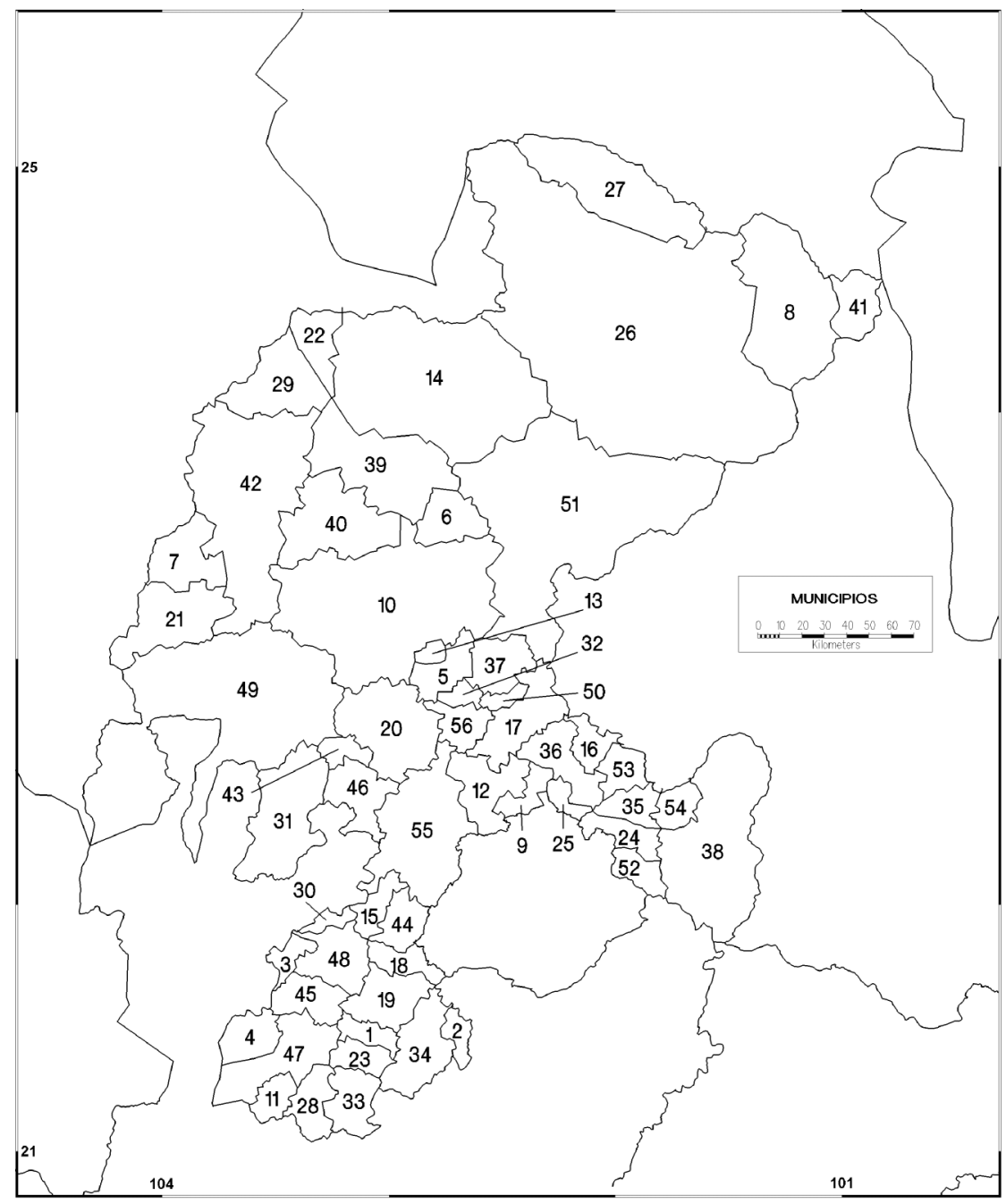

Fig. 1. División municipal del estado de Zacatecas. 1. Apozol, 2. Apulco, 3. Atolinga, 4. Benito Juárez, 5. Calera, 6. Cañitas de Felipe Pescador, 7. Chalchihuites, 8. Concepción del Oro, 9. Cuauhtémoc, 10. Fresnillo, 11. García de la Cadena, 12. Genaro Codina, 13. General Enrique Estrada, 14. General Francisco Murguía, 15. General Joaquín Amaro, 16. General Pánfilo Natera, 17. Guadalupe, 18. Huanusco, 19. Jalpa, 20. Jerez, 21. Jiménez del Teúl, 22. Juan Aldama, 23. Juchipila, 24. Loreto, 25. Luis Moya, 26. Mazapil, 27. Melchor Ocampo, 28. Mezquital del Oro, 29. Miguel Auza, 30. Momax, 31. Monte Escobedo, 32. Morelos, 33. Moyahua de Estrada, 34. Nochistlán de Mejía, 35. Noria de Angeles, 36. Ojo Caliente, 37. Pánuco, 38. Pinos, 39. Río Grande, 40. Sain Alto, 41. El Salvador, 42. Sombrerete, 43. Susticacán, 44. Tabasco, 45. Tepechitlán, 46. Tepetongo, 47. Teúl de González Ortega, 48. Tlaltenango de Sánchez Román, 49. Valparaíso, 50. Vetagrande, 51. Villa de Cos, 52. Villa García, 53. Villa González Ortega, 54. Villa Hidalgo, 55. Villanueva, 56. Zacatecas. 


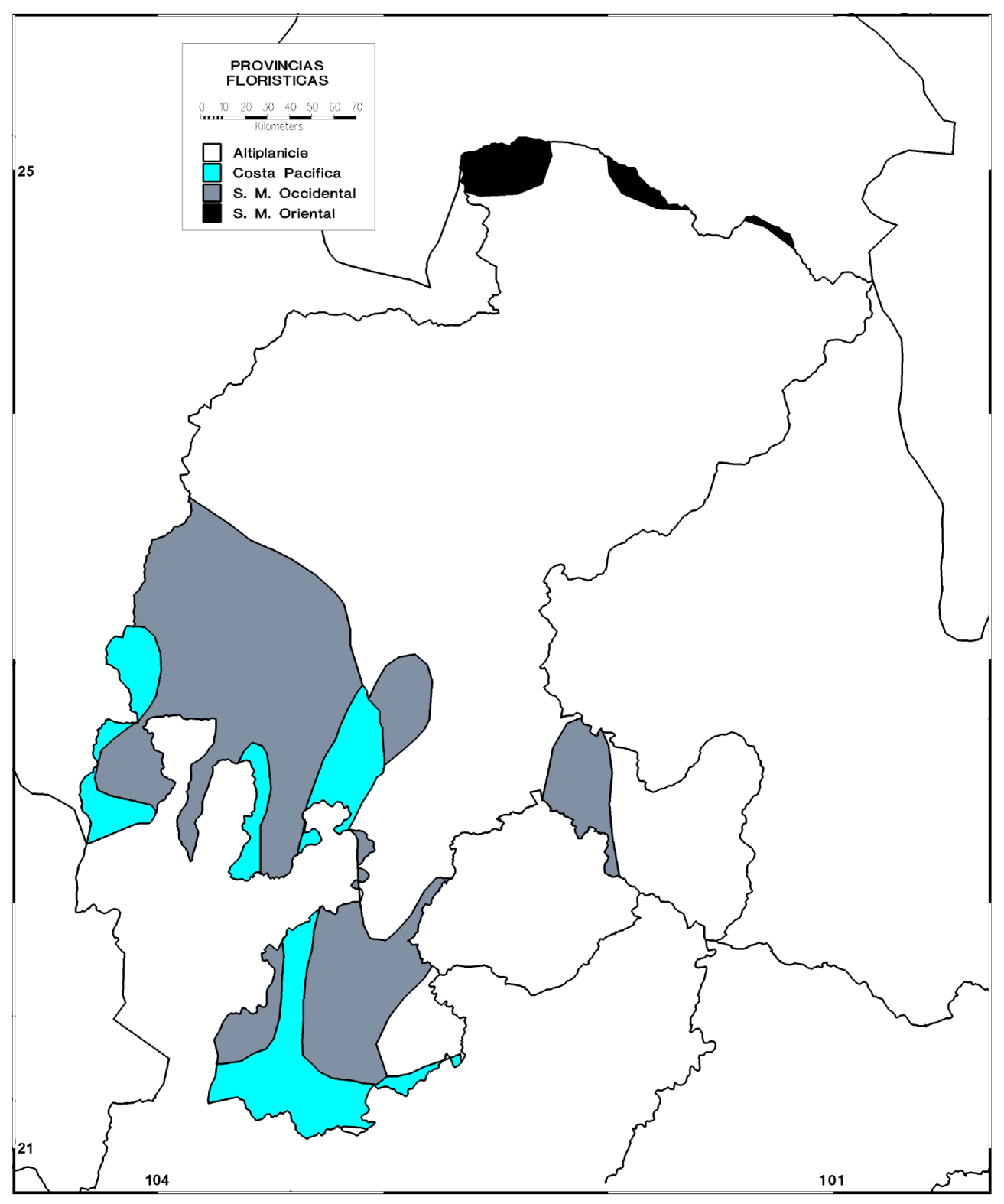

Fig. 2. División del estado de Zacatecas en provincias florísticas (basado en Rzedowski, 1992). 
Holártico y Neotropical. La tercera se ubica en la Región Xerofítica Mexicana y la cuarta en la Caribea, ambas formando parte del Reino Neotropical.

\section{Geología}

El territorio del estado se reparte en tres provincias morfotectónicas: Meseta Central, Sierra Madre Occidental y Sierra Madre Oriental (Ferrusquía, 1998). Esta división corresponde a grandes rasgos con la propuesta por el Instituto Nacional de Estadística Geografía e Informática (INEGI) que divide la superficie de la entidad en las provincias fisiográficas Eje Neovolcánico, Mesa del Centro, Sierra Madre Occidental y Sierra Madre Oriental (Anónimo, 1981; Fig. 3).

La provincia del Eje Neovolcánico está representada en el estado por una región muy pequeña, que abarca $0.84 \%$ de su superficie. Comprende la porción sur del estado, que limita con Jalisco y Aguascalientes. En esta área existen rocas ígneas extrusivas del Terciario Superior y del Cuaternario, que descansan sobre rocas extrusivas ácidas del Terciario Inferior que afloran cerca de la ciudad de Nochistlán.

La provincia de la Mesa del Centro ocupa $44 \%$ de la superficie total del estado y comprende una gran porción del centro de Zacatecas. Su constitución geológica es compleja. El núcleo está formado por rocas metamórficas y sedimentarias marinas de edad jurásica y cretácica, que en conjunto forman montañas y sierras (Ferrusquía, 1998). Estos cuerpos fueron cubiertos de manera discordante por sedimentos continentales del Terciario, que a su vez quedaron ocultos en la mayor parte de la provincia bajo depósitos aluviales del Cuaternario y sobre los cuales yacen estructuras lávicas más jóvenes (Anónimo, 1981, Ferrusquía, 1998). Los afloramientos más antiguos en esta provincia corresponden a cuerpos de rocas de edad pre-triásica localizados al noroeste de la ciudad de Zacatecas (Ferrusquía, 1998). La provincia se caracteriza por sus amplias llanuras interrumpidas por sierras dispersas. Las llanuras se encuentran entre los 2,000 y 2,200 m s.n.m. Las sierras alcanzan hasta $2,980 \mathrm{~m}$ de altitud en su punto más alto (Anónimo, 1981).

La provincia de la Sierra Madre Occidental comprende la porción sur del estado y abarca $39 \%$ de la superficie total. Este gran sistema montañoso se originó en el Terciario Inferior o Medio, al iniciarse la extrusión de los materiales volcánicos que lo integran (Anónimo, 1981). Ferrusquía (1998) señala que los macizos que la dominan pueden dividirse estratigráficamente en complejos volcánicos inferior y superior. El inferior está compuesto predominantemente por andesitas. El superior yace en discordancia sobre el inferior. Consiste de extensas secuencias ignimbrítico-silícicas, de hasta 1,000 m de espesor y abarca toda la provincia.

Localmente, los sedimentos clásticos del Terciario Tardío y del Cuaternario descansan en discordancia sobre estos dos complejos de origen ígneo, ocupando depresiones estructurales o topográficas. Fisiográficamente, la provincia consiste en una serie de sierras y mesetas volcánicas muy próximas entre sí, que coalescen en algunos sitios formando cordilleras mayores (Ferrusquía, 1998). La vertiente occidental de la Sierra se caracteriza por sus imponentes laderas escarpadas y profundos cañones excavados por afluentes del Río Santiago, mientras que el declive oriental baja de 


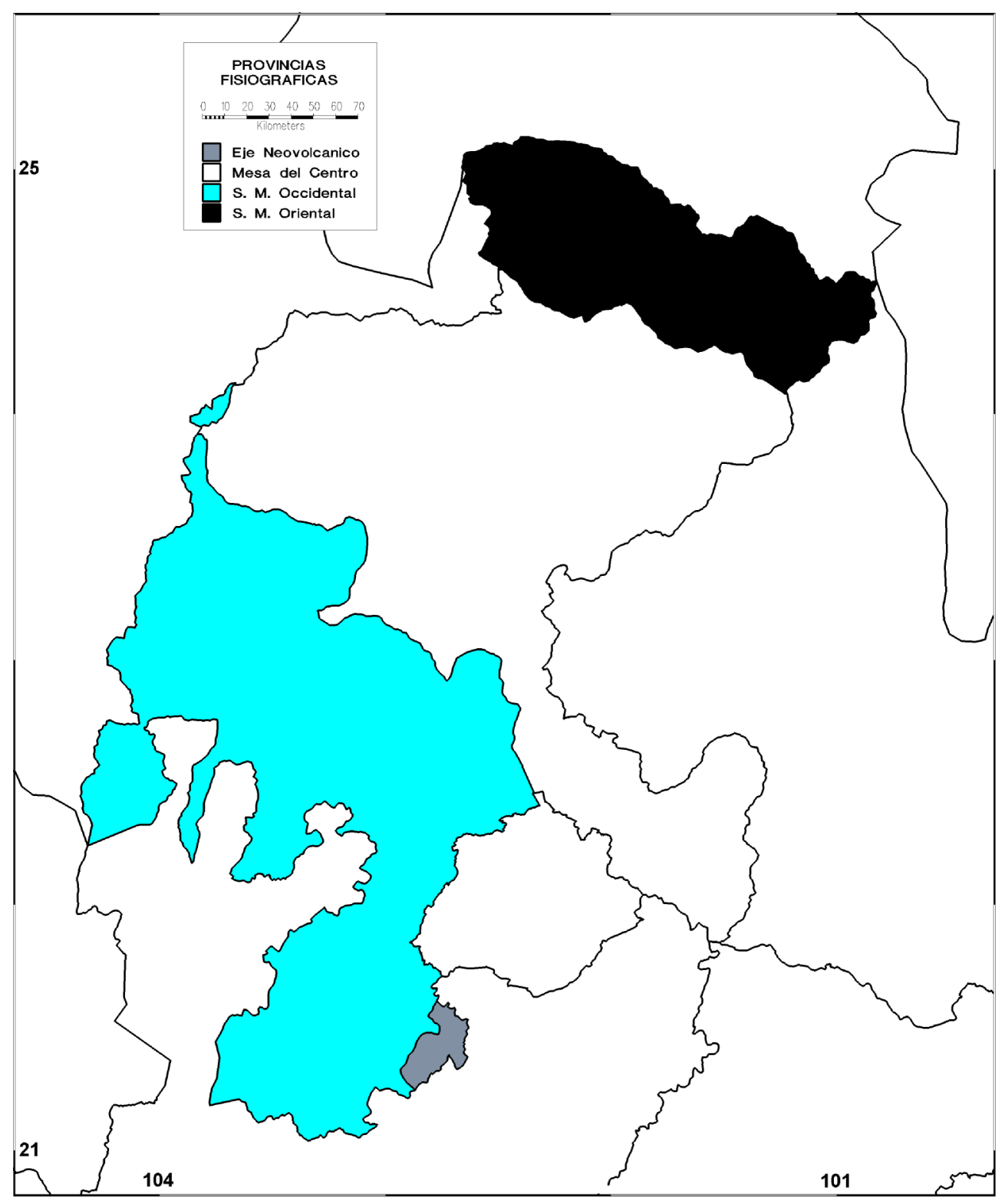

Fig. 3. División del estado de Zacatecas en provincias fisiográficas (basado en Anónimo, 1981) 
manera gradual hacia las planicies del centro. El gradiente altitudinal abarca de 950 a 3,000 m s.n.m. (Anónimo, 1981).

A la provincia de la Sierra Madre Oriental le corresponde $16 \%$ de la superficie del estado de Zacatecas y comprende su porción noreste. La región está principalmente constituida por cuerpos de rocas sedimentarias marinas del Jurásico y Cretácico, que forman cordilleras intrincadamente plegadas, separadas por cuencas intermontanas ocupadas por secuencias sedimentarias continentales cenozoicas. Contiene además un basamento pre-jurásico que incluye unidades metamórficas precámbricas y paleozoicas, así como sedimentarias del Paleozoico (Anónimo, 1981; Ferrusquía, 1998).

Zacatecas forma parte del sector transversal de la Sierra Madre Oriental y en particular de la Subprovincia de Cordones Ampliamente Espaciados. Los cordones forman por lo regular sierras altas (2,000-3,000 m s.n.m.) con orientación este-oeste; por ejemplo, las Sierra de Mazapil o la Sierra de la Candelaria (Anónimo, 1981; Ferrusquía, 1998). Las sierras están separadas por amplias planicies que pueden corresponder a valles o formar cuencas endorréicas (Ferrusquía, 1998).

Clima

De acuerdo con el sistema de clasificación climática de Köppen, modificado por García (1987) y con la Síntesis Geográfica de Zacatecas (Anónimo, 1981), en 80\% de la superficie de Zacatecas predominan los climas del grupo B (secos), en 19\% los climas del grupo C (templados) y en 1\% los del grupo A (cálido húmedos). Del B el más

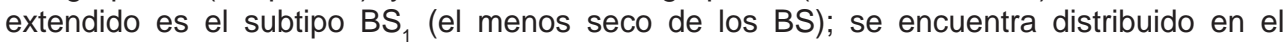
centro, norte, noreste, sureste y en una pequeña región del occidente; el segundo más extendido es el subtipo $\mathrm{BS}_{0}$ (el más seco de los BS), que se localiza en la región noreste de la entidad; finalmente, el tipo BW (muy seco o desértico) se encuentra principalmente en el extremo noreste del estado. Del grupo $\mathrm{C}$ el tipo $\mathrm{C}(\mathrm{w})$ (templado subhúmedo con lluvias en verano) es el más ampliamente distribuido, con el subtipo $C\left(w_{0}\right)$ (el más seco) ubicado en la parte oeste y el subtipo $\mathrm{C}\left(\mathrm{w}_{1}\right)$ (semiseco) principalmente en la parte sur. Del mismo grupo, el subtipo $(A) C\left(w_{0}\right)$ (el más seco de los semicálidos) se localiza en las tierras bajas del sur. Los climas del grupo $A$ se restringen a pequeñas regiones del sur de la entidad, en los límites con Jalisco.

\section{Vegetación}

En la vegetación de Zacatecas se distinguen seis diferentes tipos, siguiendo la propuesta de clasificación de Rzedowski (1978): bosque espinoso, bosque tropical caducifolio, bosque de Quercus, bosque de coníferas, matorral xerófilo y pastizal.

El bosque espinoso se presenta en forma de manchones aislados en la región central del estado (Anónimo, 1980) entre 1,500 a 2,000 m s.n.m. A este tipo de vegetación corresponden las comunidades formadas por leguminosas arbóreas con espinas y hojas compuestas de folíolos pequeños y caducos, por lo general de 4 a $10 \mathrm{~m}$ de alto. Las especies dominantes son Prosopis laevigata, Acacia farnesiana y Acacia schaffneri. Equivalen a los mezquitales que Miranda y Hernández X. (1963) ubican en la categoría de selva baja espinosa perennifolia. 
El bosque tropical caducifolio se desarrolla en suelos someros y pedregosos de laderas de cerros, principalmente en cañones y cañadas excavadas por afluentes del Río Santiago entre 1,000 a 2,200 m s.n.m. Se asocia con climas cálidos y semicálidos y dominan en su fisonomía plantas arbóreas de menos de $15 \mathrm{~m}$ de altura, que se caracterizan por la pérdida de sus hojas durante la época seca del año. Son comunes especies como Acacia farnesiana, A. pennatula, Bursera bipinnata, B. copallifera, B. fagaroides, B. multijuga, Ipomoea intrapilosa, Lysiloma acapulcense, L. microphyllum, Pithecellobium acatlense, $P$. dulce y Stenocereus queretaroensis. Este tipo de vegetación equivale a la selva baja caducifolia de la clasificación de Miranda y Hernández X. (1963) e incluye al bosque tropical deciduo y al matorral subtropical caducifolio de Guzmán y Vela (1960).

El bosque de Quercus se desarrolla entre 1,500 y 2,700 m s.n.m., en laderas y mesetas de las partes altas de las sierras, por debajo de los bosques de coníferas, con los que frecuentemente se asocia. Crece sobre diversas clases de sustrato, así como en suelos profundos de terrenos aluviales planos. En las regiones de clima templado, los bosques de Quercus están formados por árboles de 4 a $30 \mathrm{~m}$ de alto, con hojas anchas y coriáceas, que en su mayoría caen durante la época seca del año. Entre las especies más comunes destacan Quercus eduardi, Q. emoryi, Q. gentryi, Q. laeta y Q. resinosa. En las regiones con clima seco toman la forma de matorrales. Este tipo de vegetación corresponde a los encinares y chaparrales de la clasificación de Miranda y Hernández X. (1963).

El bosque de coníferas se desarrolla entre 1,800 y 3,000 m s.n.m., sobre laderas y mesetas en las partes más altas de las sierras. En Zacatecas, las comunidades vegetales que pertenecen a este tipo de vegetación son los bosques de Pinus. Se distinguen básicamente dos asociaciones diferentes, las de climas secos del centro y norte del estado, y las de climas templados subhúmedos de la región occidental. En las comunidades del centro predominan los piñonares de Pinus cembroides. Al noreste, en la región que corresponde a la Sierra Madre Oriental, son frecuentes Pinus johannis y $P$. pinceana (Anónimo, 1981). Al oeste y sur-suroeste predominan Pinus montezumae, $P$. lumholtzii, P. leiophylla y $P$. oocarpa (Anónimo, 1981). En el sur destaca la población de Pinus maximartinezii, especie endémica de Zacatecas. Este tipo de vegetación corresponde a los pinares de la clasificación de Miranda y Hernández X. (1963).

El matorral xerófilo se asocia con los climas secos que predominan en el estado; en consecuencia es el tipo de vegetación que ocupa la mayor parte de su superficie, se desarrolla entre 1,100 a 2,800 m s.n.m. Agrupa comunidades arbustivas, de las cuales se citan para Zacatecas el matorral desértico micrófilo, el matorral desértico rosetófilo y el matorral crasicaule (Rzedowski, 1978). El matorral desértico micrófilo ocupa las extensas llanuras del noreste y algunas áreas del norte y sur del estado. Incluye comunidades donde las especies que imprimen el carácter fisonómico son arbustos de hoja o foliolo pequeño, sobre todo de los géneros Acacia, Condalia, Flourensia, Koeberlinia, Larrea, Lycium, Mimosa y Prosopis. Comprende el matorral inerme parvifolio y el matorral espinoso con espinas laterales de la clasificación de Miranda y Hernández X. (1963). El matorral desértico rosetófilo se localiza principalmente en el noreste, en donde ocupa las laderas de cerros calizos y las porciones superiores de los abanicos aluviales contiguos a los mismos. Agrupa las comunidades donde predominan especies arbustivas o subarbustivas con las hojas dispuestas en forma de roseta. Las rosetas pueden 
ser basales (Agave) o en el extremo de un tallo manifiesto (Yucca). Este tipo de vegetación comprende los izotales, los magueyales, los lechuguillales y los guapillales (crasirosulifolios espinosos) de Miranda y Hernández X. (1963). El matorral crasicaule se localiza en regiones del sureste, centro y norte del estado, preferentemente en suelos someros de laderas de cerros de origen volcánico. Agrupa todas las comunidades arbustivas de clima árido y semiárido en los que el papel importante corresponde a plantas conspicuas de tallo suculento (Rzedowski, 1978). En Zacatecas las comunidades que pertenecen a este tipo de vegetación presentan como cubierta vegetal un matorral de Opuntia, siendo las especies dominantes $O$. streptacantha y O. leucotricha (Rzedowski, 1957). Corresponde a las nopaleras del sistema de clasificación de Miranda y Hernández X. (1963).

El pastizal reúne convencionalmente a las comunidades vegetales donde el papel preponderante corresponde a las gramíneas (Rzedowski, 1978). Los pastizales se encuentran en prácticamente todo el estado (Anónimo, 1980) en altitudes que varían de 1,700 a 2,600 m s.n.m.; los más típicos son los pastizales de Bouteloua, que se ubican en la zona de transición entre los bosques y el matorral xerófilo. En las partes medias y altas de la Sierra Madre Occidental se encuentran pastizales amacollados de Muhlenbergia, Elyonurus o Schizachyrium. En suelos con alto contenido de sales se desarrollan los de Sporobolus airoides, Distichlis spicata y Eragrostis obtusiflora. Este tipo de vegetación incluye la pradera de Guzmán y Vela (1960) y el zacatal de Rzedowski y McVaugh (1965).

\section{MATERIALES Y MÉTODO}

Se recolectaron especímenes de Asteraceae en floración de 1997 a 1999, en las cuatro estaciones del año. Se registraron las coordenadas geográficas de las localidades con la ayuda de un geoposicionador, lo que sirvió para documentar gráficamente los patrones de distribución geográfica de las especies. En total se realizaron 116 excursiones de recolecta en el estado; se visitaron 685 localidades representativas de todos los tipos de vegetación propios de la entidad (Fig. 4) y se integró una colección de 4,092 ejemplares. El primer juego de especímenes quedó depositado en el Herbario de la Universidad Autónoma de Zacatecas (HUAZ) y el segundo en el Herbario Nacional (MEXU) del Instituto de Biología de la Universidad Nacional Autónoma de México.

Todos los ejemplares recolectados se identificaron críticamente, privilegiando el uso de floras, monografías y revisiones (por ejemplo Anderberg, 1991; Bremer, 1994; Bremer y Humphries, 1993; King y Robinson, 1987; McVaugh, 1984; Strother, 1969; Strother, 1986; Turner, 1996; Turner, 1997). Los ejemplares identificados se cotejaron con las descripciones que proporcionó la bibliografía consultada y posteriormente se compararon con los especímenes depositados en MEXU. Siempre que se juzgó apropiado, se usó el nombre de la especie de más reciente publicación y en los casos de discrepancia, después del binomio aceptado, se citó entre corchetes el nombre que reconoce McVaugh (1984) (ver anexo), la obra más relevante de información para la familia y de amplia consulta en la región de estudio. Los nombres de los autores de las especies están abreviados siguiendo a Villaseñor (2001).

Las especies fueron ubicadas en las tribus utilizando los esquemas de clasificación más recientes. Se siguió en gran medida lo sintetizado en la obra de Bremer (1994), excepto en aquellos casos donde hubiera un estudio taxonómico más específico. 
Tal es el caso por ejemplo de la tribu Anthemideae, donde se prefirió seguir a Bremer y Humphries (1993), o a Turner $(1996,1997)$ para las tribus Eupatorieae y Tageteae, aunque con las modificaciones genéricas propuestas por King \& Robinson (1987) para las Eupatorieae y Strother (1986) para las Tageteae.

Por otra parte, se identificaron igualmente los ejemplares de Asteraceae recolectados en el estado y que se conservan en el MEXU, en el Herbario de la Escuela Nacional de Ciencias Biológicas del Instituto Politécnico Nacional (ENCB) y en el Herbario del Colegio de Postgraduados (CHAPA).

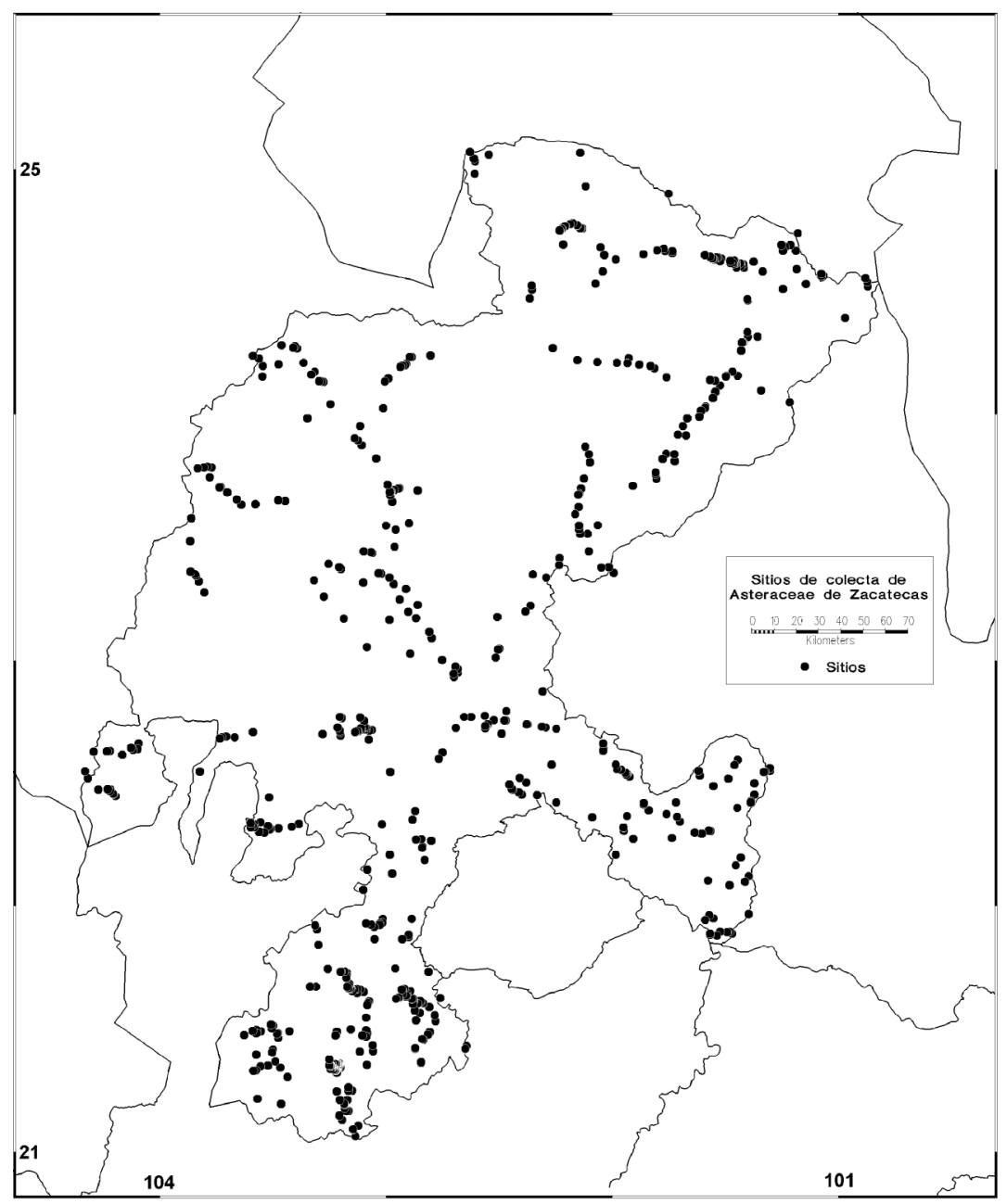

Fig. 4. Localidades de muestreo y recolección en Zacatecas. Se registraron 685 sitios con coordenadas geográficas precisas, donde se recolectaron 4,092 especímenes. 
En total se revisaron 4,714 especímenes que documentan la flora sinanterológica del estado de Zacatecas.

\section{RESULTADOS}

La flora sinanterológica conocida de Zacatecas se compone de 456 especies, en este conjunto se reconocen 119 entidades subespecíficas (variedades o subespecies). Las especies están repartidas en 141 géneros y 14 tribus. Por su número de especies (Cuadro 1), la tribu Heliantheae (149) es la más importante, seguida por Eupatorieae (96) y Astereae (68), mientras que las otras 11 tribus contribuyen con 30 o menos a la riqueza total. Un patrón análogo de diversidad se observa si se toman en cuenta únicamente a las especies de distribución restringida al territorio nacional y registradas en el estado. Las mismas tribus reportan el mayor número de especies endémicas.

En el estado existen 12 géneros con 10 o más especies (Cuadro 2); en ellos se concentra $39 \%$ de la diversidad total. Por otra parte, 65 géneros cuentan con una sola especie y 25 registran únicamente dos (Fig. 5). Lo anterior denota el patrón generalizado de pocos géneros con muchas especies y muchos géneros con una o pocas especies.

Con base en la información disponible, las Asteraceae superan en número de especies a otras familias que participan en la flora fanerogámica del estado de Zacatecas (Cuadro 3). Sus miembros rebasan en número a las Poaceae y a las Fabaceae, las otras dos familias más importantes en la flora estatal.

Cuadro 1. Número de géneros y especies de Asteraceae por tribu registrados en Zacatecas. Se incluye también el número de especies endémicas de México registradas en el estado.

\begin{tabular}{|l|c|c|c|}
\hline Tribu & Géneros & Especies & Especies endémicas \\
\hline Anthemideae & 3 & 5 & 2 \\
Astereae & 22 & 68 & 28 \\
Cardueae & 2 & 8 & 4 \\
Eupatorieae & 17 & 96 & 63 \\
Gnaphalieae & 2 & 15 & 4 \\
Helenieae & 14 & 25 & 13 \\
Heliantheae & 50 & 149 & 78 \\
Lactuceae & 6 & 9 & 1 \\
Liabeae & 1 & 2 & 2 \\
Mutisieae & 5 & 1 & 13 \\
Plucheae & 1 & 25 & 0 \\
Senecioneae & 7 & 25 & 9 \\
Tageteae & 8 & 8 & 8 \\
Vernonieae & 3 & 456 & 247 \\
& 141 & & 9 \\
\hline
\end{tabular}


Cuadro 2. Géneros de Asteraceae con 10 o más especies en Zacatecas. Se incluye también el número de especies endémicas de México registradas en el estado.

\begin{tabular}{|l|c|c|}
\hline Género & Especies & Especies endémicas \\
\hline Stevia & 27 & 19 \\
Ageratina & 23 & 18 \\
Brickellia & 21 & 14 \\
Viguiera & 15 & 12 \\
Verbesina & 14 & 12 \\
Pseudognaphalium & 13 & 4 \\
Acourtia & 12 & 9 \\
Bidens & 12 & 5 \\
Cosmos & 11 & 7 \\
Erigeron & 11 & 6 \\
Baccharis & 10 & 4 \\
Psacalium & 10 & 10 \\
\hline
\end{tabular}

Cuadro 3. Las cinco familias con mayor número de especies en el estado de Zacatecas (Balleza, 1992; Villaseñor, datos no publicados).

\begin{tabular}{|l|c|c|}
\hline Familia & Géneros & Especies \\
\hline Asteraceae & 141 & 456 \\
Poaceae & 84 & 292 \\
Fabaceae & 35 & 149 \\
Cactaceae & 22 & 90 \\
Mimosaceae & 12 & 49 \\
\hline
\end{tabular}

Patrones de diversidad

Los tipos de vegetación con mayor riqueza de géneros y especies de Asteraceae son el bosque de Quercus, el matorral xerófilo y el bosque de coníferas (Cuadro 4). El bosque espinoso es el que registró el menor número de taxa de esta familia.

Las comunidades de clima templado (bosques de encinos y de coníferas) registraron también la cantidad más grande de especies de distribución geográfica restringida al territorio nacional.

A nivel de provincias fisiográficas de Zacatecas, la de la Sierra Madre Occidental alberga el mayor número de géneros y especies. En orden descendente le siguen la Mesa del Centro, la Sierra Madre Oriental y el Eje Neovolcánico (Cuadro 5).

La diversidad de especies de la familia es mayor en los estados ubicados al sur y al oeste de Zacatecas, que en los que limitan al norte y al este. Por entidad federativa, la riqueza de Asteraceae de Zacatecas es muy similar a la de Coahuila y Nayarit, a pesar de que existen grandes diferencias entre la superficie total de cada una de las entidades (Cuadro 6). 


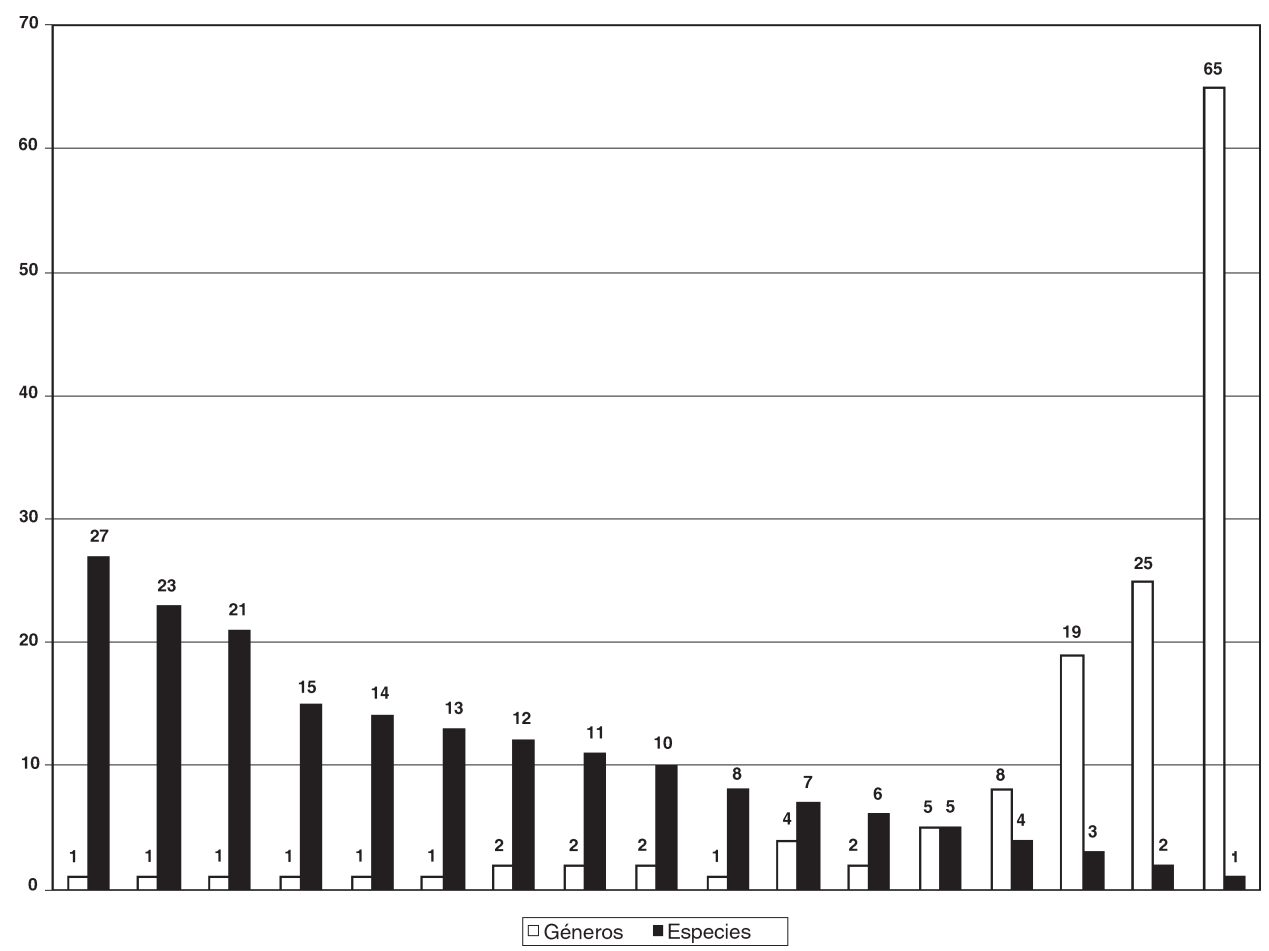

Fig. 5. Correspondencias entre el número de géneros de Asteraceae y el número de especies que contienen.

Se ha documentado ampliamente el hecho de que el número de taxa se incrementa al aumentar el área (ver por ejemplo Rosenzweig, 1995). En consecuencia, para tener una mejor idea de la diversidad de Asteraceae existente en Zacatecas y los estados vecinos, se hizo un ajuste de la riqueza de especies de cada estado, dividiendo el número de especies por el área $\left(\mathrm{km}^{2}\right)$ transformada a una escala logarítmica. Un procedimiento idéntico se hizo evaluando los tipos de vegetación y las provincias fisiográficas de Zacatecas (Cuadro 7).

Los estados de Coahuila, Zacatecas y Nayarit poseen una cantidad de especies similar. Sin embargo, cuando el número de especies es ajustado por el logaritmo del área ("densidad de especies") se observa que Zacatecas tiene mayor riqueza por unidad de área que Coahuila, pero menor que Nayarit (Cuadro 7).

Por tipo de vegetación, los bosques de coníferas y de Quercus y el matorral xerófilo registran más de 200 especies (Cuadro 7), aunque el área ocupada por este último es casi 12 veces más grande que la correspondiente a las otras dos comunidades. Cuando 
se estima la diversidad relativa, se observa que el bosque de encinos contiene una mayor riqueza por unidad de área. En el mismo contexto el pastizal (ubicado en "otros" en el Cuadro 7) sobresale por su número de especies, confinando al matorral xerófilo a un menor nivel de importancia, no obstante la gran extensión que ocupa.

Cuadro 4. Distribución de géneros y especies de Asteraceae por tipo de vegetación en Zacatecas. Se incluye también el número de especies endémicas de México registradas en el estado.

\begin{tabular}{|l|c|c|c|}
\hline Tipo de vegetación & Géneros & Especies & Especies endémicas \\
\hline Bosque de coníferas & 96 & 206 & 113 \\
Bosque de Quercus & 91 & 264 & 151 \\
Bosque espinoso & 39 & 49 & 17 \\
Bosque tropical & & 146 & 68 \\
caducifolio & 74 & 222 & 94 \\
Matorral xerófilo & 100 & 181 & 79 \\
Pastizal & 96 & & \\
\hline
\end{tabular}

Cuadro 5. Distribución de géneros y especies de Asteraceae por provincia fisiográfica en Zacatecas. Se incluye también el número de especies endémicas de México registradas en el estado.

\begin{tabular}{|l|c|c|c|}
\hline Provincia fisiográfica & Géneros & Especies & Especies endémicas \\
\hline Eje Neovolcánico & 45 & 83 & 31 \\
Mesa del Centro & 90 & 191 & 77 \\
Sierra Madre Occidental & 124 & 368 & 211 \\
Sierra Madre Oriental & 66 & 107 & 38 \\
\hline
\end{tabular}

Cuadro 6. Géneros, especies y taxa infraespecificos de Asteraceae registrados para Zacatecas y estados vecinos (Ortiz-Bermúdez, et al., 1998; Villarreal, et al. 1996; Villaseñor, 1993).

\begin{tabular}{|l|c|c|c|c|}
\hline Estado & Superficie $\left(\mathrm{km}^{2}\right)$ & Géneros & Especies & $\begin{array}{c}\text { Taxa } \\
\text { infraespecíficos }\end{array}$ \\
\hline Zacatecas & 74,668 & 141 & 456 & 119 \\
Aguascalientes & 5,471 & 81 & 178 & 6 \\
Coahuila & 149,982 & 143 & 490 & 32 \\
Durango & 123,181 & 149 & 602 & 38 \\
Jalisco & 80,836 & 157 & 750 & 55 \\
Nayarit & 27,865 & 122 & 447 & 24 \\
\hline
\end{tabular}


Cuadro 7. Diversidad relativa(relación especies/logaritmo de la superficie) de Asteraceae en Zacatecas y estados vecinos. Se incluye también la diversidad relativa en los principales tipos de vegetación y en las provincias fisiográficas presentes en el estado de Zacatecas.

\begin{tabular}{|l|c|c|c|}
\hline & Superficie $\left(\mathrm{km}^{2}\right)$ & Especies & Diversidad \\
\hline Zacatecas & 74,668 & 456 & 93.6 \\
Aguascalientes & 5,471 & 178 & 47.6 \\
Coahuila & 149,982 & 490 & 94.6 \\
Durango & 123,181 & 602 & 118.3 \\
Jalisco & 80,836 & 750 & 152.8 \\
Nayarit & 27,865 & 447 & 100.6 \\
Matorral xerófilo & & 242 & 52.1 \\
Bosque de Quercus & $43,760.7$ & 290 & 81.1 \\
Bosque de coníferas & $3,750.5$ & 210 & 59.3 \\
Bosque tropical caducifolio & $3,457.5$ & 151 & 54.7 \\
Otros & 578.7 & 186 & 66.2 \\
& 644.6 & 83 & 29.8 \\
Eje Neovolcánico & & 192 & 42.6 \\
Mesa del Centro & 615.3 & 368 & 82.6 \\
Sierra Madre Occidental & 32,230 & 108 & 26.5 \\
Sierra Madre Oriental & $28,568.3$ & $11,720.3$ & \\
\hline
\end{tabular}

La Sierra Madre Occidental, sin ser la provincia con mayor superficie en el estado, destaca igualmente por su mayor riqueza de especies y por la mayor densidad de las mismas, casi duplicando en número a la provincia que ocupa el segundo lugar, la Mesa del Centro.

Sobresale también el Eje Neovolcánico, provincia que ocupa una pequeña fracción del estado $\left(615 \mathrm{~km}^{2}\right)$, pero que presenta una densidad de especies parecida a la de la Sierra Madre Oriental, que le dobla en extensión.

\section{Afinidades geográficas}

De las 456 especies de Asteraceae que se conocen en Zacatecas, 247 (54\%) son endémicas de México. Por otra parte, cuatro especies y una variedad aparentemente restringen su área de distribución al estado (Ageratina jalpana, Erigeron zacatensis, Perityle glaucescens, Stevia zacatecana y Verbesina parviflora var. zacatecana). El área de Erigeron zacatensis se ubica en la Mesa del Centro y las otras tres especies limitan su distribución a la Sierra Madre Occidental, particularmente en la cordillera conocida regionalmente como Sierra de Morones. El porcentaje de endemismo a nivel estatal es bajo $(0.87 \%)$, comparado con los valores que Villaseñor (1993) señala para otros estados vecinos, como Coahuila (12\%), Durango (11.3\%), Jalisco (11\%) o San Luis Potosí (7.4\%).

Tomando como referencia una región más amplia, que incluye a los estados que limitan con Zacatecas, el número de endemismos se incrementa a 30, cantidad que representa $6 \%$ del total estatal. Durango y Jalisco son las entidades con las que Zacatecas comparte el mayor número de endemismos vecinales (Cuadro 8). 
Cuadro 8. Distribución de las especies endémicas de Zacatecas y estados vecinos. 1 = Zacatecas; 2 = Zacatecas, Coahuila; 3 = Zacatecas, Durango; 4 = Zacatecas, Jalisco; 5 = Zacatecas, Nayarit; $6=$ Zacatecas, Aguascalientes, Jalisco; 7 = Zacatecas, Jalisco, Nayarit; 8 = Zacatecas, Nuevo León, San Luis Potosí.

\begin{tabular}{|c|c|c|c|c|c|c|c|c|}
\hline Especies endémicas & 1 & 2 & 3 & 4 & 5 & 6 & 7 & 8 \\
\hline $\begin{array}{l}\text { Acourtia longifolia } \\
\text { Ageratina astillera } \\
\text { Ageratina jalpana } \\
\text { Ageratina leptodictyon } \\
\text { Brickellia robinsoniana } \\
\text { Coreopsis cyclocarpa var. } \\
\text { cyclocarpa } \\
\text { Cosmos concolor } \\
\text { Cosmos landii } \\
\text { Cosmos sessilis } \\
\text { Erigeron exilis } \\
\text { Erigeron zacatensis } \\
\text { Flourensia dentata } \\
\text { Pectis propetes } \\
\text { Perityle feddemae } \\
\text { Perityle glaucescens } \\
\text { Perityle trichodonta } \\
\text { Perymenium subsquarrosum } \\
\text { Pinaropappus diguetii } \\
\text { Roldana pennellii var. durangensis } \\
\text { Senecio pseudopicridis } \\
\text { Stevia rzedowskii } \\
\text { Stevia zacatecana } \\
\text { Tridax petrophila } \\
\text { Verbesina hispida } \\
\text { Verbesina parviflora } \\
\text { var. zacatecana } \\
\text { Viguiera hypargyrea } \\
\text { Viguiera palmeri var. rzedowskii } \\
\text { Viguiera schultzii } \\
\text { Wedelia grayi } \\
\text { Xylothamia parrasana }\end{array}$ & $\mathrm{X}$ & $x$ & $x$ & $\begin{array}{l}X \\
X \\
X\end{array}$ & $x$ & $X$ & $\begin{array}{l}X \\
X\end{array}$ & $x$ \\
\hline
\end{tabular}

Entre las especies no endémicas de México, predominan las que existen también en Norte y Centroamérica. Así, de las 209 que se distribuyen más allá de las fronteras del país, 88 amplían su área hacia los Estados Unidos y 50 a Centroamérica. Por otra parte, 34 especies viven tanto en Norte como Centroamérica, 12 se conocen asimismo de Sudamérica, 14 presentan una distribución amplia, prácticamente en todo el Continente Americano y 11 se registran también del Viejo Mundo. 
Es importante enfatizar que 191 especies de Asteraceae de Zacatecas (42\% del total) califican como malezas, de acuerdo con Villaseñor y Espinosa (1998). De tal contingente de plantas arvenses o ruderales, 59 son endémicas de México.

Formas de vida

Entre las Asteraceae de Zacatecas predominan las hierbas, tanto anuales como perennes (Cuadro 9). Después del hábito herbáceo, la forma de vida más importante es la arbustiva. Únicamente se registran dos especies con el hábito arbóreo o arborescente (ver anexo).

Cuadro 9. Distribución de especies de Asteraceae por forma de vida en Zacatecas. Se incluye también el número de especies endémicas de México registradas en el estado.

\begin{tabular}{|l|c|c|}
\hline Forma de vida & Especies & Especies endémicas \\
\hline Árboles & 2 & 2 \\
Arbustos & 84 & 52 \\
Hierbas anuales & 124 & 39 \\
Hierbas perennes & 245 & 154 \\
\hline
\end{tabular}

\section{DISCUSIÓN}

La familia Asteraceae es la más diversa de todas las familias de plantas con flores en el estado de Zacatecas. Supera en número de especies a otras familias también muy diversas en ambientes como los encontrados en el estado, tales como Poaceae y Fabaceae (Villaseñor, datos no publicados).

Entre las Asteraceae de Zacatecas, las tribus Heliantheae, Eupatorieae y Astereae son las más ricas en géneros y especies, tanto si se considera la diversidad total, 0 únicamente las especies endémicas de México. Por tipo de vegetación, el bosque de Quercus, el matorral xerófilo, el bosque de coníferas y el pastizal registran más géneros y especies de Asteraceae que el bosque tropical caducifolio y el bosque espinoso. Asimismo, la forma de vida con mayor número de especies es la herbácea, dominando las hierbas perennes entre el componente endémico. Estos patrones son similares a los que se han encontrado para las Asteraceae de todo el país (Rzedowski, 1972).

Los géneros con más especies en Zacatecas pertenecen a las tribus mejor representadas en su flora sinanterológica: Stevia (27 especies), Ageratina (23) y Brickellia (21) se agrupan en la tribu Eupatorieae; Viguiera (15) y Verbesina (14) en Heliantheae; Erigeron (11) y Baccharis (10) en Astereae, y Psacalium (10) en Senecioneae. Turner y Nesom (1998) obtienen un resultado similar para el conjunto de asteráceas mexicanas.

La riqueza florística del estado de Zacatecas es, en gran parte, resultado de la convergencia en el estado de las provincias fisiográficas Sierra Madre Occidental, Sierra 
Madre Oriental, Mesa del Centro y Eje Neovolcánico y de la conjunción en su territorio de los reinos biogeográficos Holártico y Neotropical. La distribución de las especies de Asteraceae por provincia fisiográfica en Zacatecas, refleja el predominio de la Sierra Madre Occidental y la Mesa del Centro, que en conjunto abarcan $83 \%$ de su territorio; pero también apoya la idea de que tanto las zonas montañosas como las zonas áridas y semiáridas de México representan las principales áreas de diversificación de esta familia (Rzedowski, 1972).

La provincia fisiográfica Sierra Madre Occidental corresponde en general, a la provincia florística del mismo nombre. En la flora de esta región el elemento holártico prevalece ligeramente sobre el neotropical y sobre el autóctono (Rzedowski, 1978). En Zacatecas, esta área registra el valor más alto de densidad de especies de Asteraceae y el mayor número de especies endémicas de esta familia en México. Por otra parte, la Mesa del Centro corresponde a la provincia florística denominada Altiplanicie. En la flora de esta región predomina el componente de afinidad neotropical sobre el holártico (Rzedowski, 1978). La densidad de especies de Asteraceae y la riqueza de entidades endémicas de México en esta superficie del estado ocupan el segundo lugar, pero son sensiblemente menores que las que se registran para la Sierra Madre Occidental. Estos resultados ayudan a explicar los patrones de distribución geográfica de las Asteraceae de Zacatecas y apoyan las ideas que sustentan que la Sierra Madre Occidental ha sido un centro importante de evolución de la mayoría de los taxa mexicanos, especialmente los de tribus grandes como Eupatorieae, Heliantheae y Senecioneae, papel que comparte con la Sierra Madre del Sur y el Eje Neovolcánico (Turner y Nesom, 1998). La disparidad que existe entre el porcentaje de especies de Asteraceae endémicas de Zacatecas y el que se registra para los estados vecinos, seguramente es resultado de la mayor presencia de la Sierra Madre Occidental en Durango y de la Sierra Madre del Sur y del Eje Neovolcánico en Nayarit y Jalisco. La abundancia de suelos yesosos y la existencia de refugios de la flora durante el Pleistoceno en Coahuila y San Luis Potosí (Rzedowski, 1991) explican también esta disparidad.

De acuerdo con el porcentaje de especies endémicas, Zacatecas es importante para la conservación de las plantas que restringen su área de distribución a su territorio, a los estados circunvecinos y al país. La información presentada en este trabajo es básica para diseñar estrategias de conservación para proteger estas rarezas.

\section{AGRADECIMIENTOS}

Este trabajo forma parte del proyecto de tesis doctoral del primer autor y fue posible gracias al apoyo económico que para el estudio de la biogeografía y conservación de las Asteraceae en el estado de Zacatecas fue otorgado por el CONACYT (28575N). José de Jesús Balleza agradece a Miguel Adame G. y a David Enríquez E. su valiosa colaboración en la recolección del material botánico. El segundo autor da gracias al Instituto de Biología de la Universidad Nacional Autónoma de México por las facilidades para desarrollar una estancia sabática asociado a la Universidad Autónoma de Zacatecas, donde parte de este trabajo fue llevado a cabo. Igualmente agradece al Consejo Nacional de Ciencia y Tecnología el apoyo económico, a través de una beca para estancias sabáticas. 


\section{LITERATURA CITADA}

Anderberg, A. A. 1991. Taxonomy and phylogeny of the tribe Gnaphalieae (Asteraceae). Opera Bot. 104: $1-195$.

Anónimo. 1980. Coeficientes de agostadero de la República Mexicana. Estados de Zacatecas y Aguascalientes. 2 vols. Comisión Técnico Consultiva para la determinación Regional de los Coeficientes de Agostadero, Secretaría de Agricultura y Recursos Hidráulicos. México D.F. $267 \mathrm{pp}$.

Anónimo. 1981. Síntesis geográfica de Zacatecas. Secretaría de Programación y Presupuesto. Coordinación General de los Servicios Nacionales de Estadística, Geografía e Informática. México, D.F. 222 pp.

Balleza, J. J. 1992. Gramíneas del estado de Zacatecas. Catálogo de especies y clave para géneros. Tesis de Maestría en Ciencias. Colegio de Postgraduados. Montecillos, Estado de México. 108 pp.

Bremer, K. 1994. Asteraceae. Cladistics \& classification. Timber Press. Portland, Oregon. 752 pp.

Bremer, K. y C. J. Humphries. 1993. Generic monograph of the Asteraceae-Anthemideae. Bull. Nat. Hist. Mus. Lond. (Bot.) 23: 71-177.

Ferrusquía, I. 1998. Geología de México: una sinopsis. In: Ramamoorthy, T. P., R. Bye, A. Lot y J. Fa. (eds.). Diversidad biológica de México: orígenes y distribución. Universidad Nacional Autónoma de México. México, D.F. pp. 3-108.

García, E. 1987. Modificaciones al sistema de clasificación climática de Köppen. 4a. edición particular. México, D.F. 217 pp.

Guzmán, H. y L. Vela. 1960. Contribución al conocimiento de la vegetación del suroeste del estado de Zacatecas. Bol. Soc. Bot. México 25: 46-60.

King, R. M. y H. Robinson. 1987. The genera of the Eupatorieae (Asteraceae). Monographs in Systematic Botany. Missouri Bot. Gard. 22: 1-581.

McVaugh, R. 1984. Compositae. In: Anderson, W. R. (ed.). Flora Novo Galiciana. The University of Michigan Press. Ann Arbor. Vol. 12. 1157 pp.

Miranda, F. y E. Hernández X. 1963. Los tipos de vegetación de México y sus clasificación. Bol. Soc. Bot. México 28: 29-179.

Ortiz-Bermúdez, E. J. L. Villaseñor y O. Téllez. 1998. La familia Asteraceae en el estado de Nayarit (México). Acta Bot. Mex. 44: 25-57.

Rosenzweig, M. L. 1995. Species diversity in space and time. Cambridge University Press. Cambridge. $436 \mathrm{pp}$.

Rzedowski, J. 1957. Vegetación de las partes áridas de los estados de San Luis Potosí y Zacatecas. Revista Soc. Mex. Hist. Nat. 18: 49-101.

Rzedowski, J. 1972. Contribuciones a la fitogeografía florística e histórica de México. III. Algunas tendencias en la distribución geográfica y ecológica de las Compositae mexicanas. Ciencia (México) 27: 123-132.

Rzedowski, J. 1978. Vegetación de México. Limusa. México, D.F. 432 pp.

Rzedowski, J. 1991. El endemismo en la flora fanerogámica mexicana: una apreciación analítica preliminar. Acta Bot. Mex. 15: 47-64.

Rzedowski, J. 1992. Tópicos fitogeográficos (provincias, matorral xerófilo y cactáceas). Mapa IV.8.3 Naturaleza. In: Atlas Nacional de México. Instituto de Geografía. Universidad Nacional Autónoma de México. México, D.F.

Rzedowski, J. y R. McVaugh. 1965. La vegetación de Nueva Galicia. Contr. Univ. Michigan Herb. 9: $1-123$.

Strother, J. L. 1969. Systematics of Dyssodia Cavanilles (Compositae: Tageteae). Univ. Calif. Publ. Bot. 48: 1-88.

Strother, J. L. 1986. Renovation of Dyssodia (Compositae: Tageteae). Sida 11: 371-378. 
Turner, B. L. 1996. The comps of Mexico. A systematic account of the family Asteraceae. Vol. 6. Tageteae and Anthemideae. Phytologia Memoirs 10: 1-93.

Turner, B. L. 1997. The comps of Mexico. A systematic account of the family Asteraceae. Vol. 1. Eupatorieae. Phytologia Memoirs 11: 1-272.

Turner, B. L. y G. L. Nesom, 1998. Biogeografía, diversidad y situación de peligro o amenaza de Asteraceae de México. In: Ramamoorthy T. P., R. Bye, A. Lot y J. Fa. (eds.). Diversidad biológica de México: orígenes y distribución. Universidad Nacional Autónoma de México. México, D.F. pp. 545-561.

Villarreal, J. A., J. Valdés y J. L. Villaseñor. 1996. Corologia de las Asteraceae de Coahuila, México. Acta Bot. Mex. 36: 29-42.

Villaseñor, J. L. 1993. La familia Asteraceae en México. Revista Soc. Mex. Hist. Nat. Vol. Esp. (XLIV): 117-124.

Villaseñor, J. L. y F. Espinosa G. 1998. Catálogo de malezas de México. Universidad Nacional Autónoma de México y Fondo de Cultura Económica. México, D.F. 449 pp.

Villaseñor, J. L. 2001. Catálogo de autores de plantas vasculares de México. Instituto de Biología, Universidad Nacional Autónoma de México y Comisión Nacional para el Conocimiento y Uso de la Biodiversidad. México, D.F. 40 pp. 
Balleza y Villaseñor: La Familia Asteraceae en el Estado de Zacatecas

ANEXO

Catálogo de Asteraceae del estado de Zacatecas, México

Entre corchetes se anota el nombre aceptado por McVaugh (1984). Con un asterisco se indica que la especie restringe su área de distribución a México. La forma de vida de la especie se indica de acuerdo con la siguiente clave: HA = Hierba anual; HP = hierba perenne incluyendo sufrútice; AR = Arbusto y $\mathrm{A}=$ Árbol. Únicamente se cita un ejemplar representativo por municipio.

Achillea millefolium L. [=Achillea lanulosa Nutt.]

HP. Jerez, Balleza 6558 (HUAZ).

Acmella oppositifolia (Lam.) R. K. Jansen var. oppositifolia [=Spilanthes oppositifolia (Lam.) D'Arcy] HP. Jalpa, Balleza 5348 (HUAZ).

Acmella radicans (Jacq.) R. K. Jansen var. radicans [=Spilanthes alba L'Her.]

HA. Juchipila, Balleza 7790 (HUAZ); Moyahua de Estrada, Enríquez 1350 (HUAZ).

${ }^{*}$ Acourtia fruticosa (La Llave) B. L. Turner [=Perezia rigida (DC.) A. Gray]

HP. Cuauhtémoc, Balleza 10389 (HUAZ); García de la Cadena, Balleza 7300 (HUAZ); General Francisco Murguía, Balleza 6948 (HUAZ); Jalpa, Balleza 7544 (HUAZ); Juchipila, Balleza 11809 (HUAZ); Monte Escobedo, Balleza 7417 (HUAZ); Río Grande, Balleza 5723 (HUAZ); Teúl de González Ortega, Balleza 8052 (HUAZ); Valparaíso, Balleza 7528 (HUAZ); Villanueva, Balleza 3597 (HUAZ).

*Acourtia glomeriflora (A. Gray) Reveal \& R. M. King [=Perezia glomeriflora (A. Gray) McVaugh] HP. Jalpa, Balleza 7976 (HUAZ); Juchipila, Balleza 8148 (HUAZ); Moyahua de Estrada, Enríquez 1419 (HUAZ).

${ }^{*}$ Acourtia grandifolia (S. Watson) Reveal \& R. M. King [=Perezia grandifolia S. Watson] HP. Jerez, Balleza 7078a (HUAZ).

${ }^{*}$ Acourtia longifolia (S. F. Blake) Reveal \& R. M. King [=Perezia longifolia S. F. Blake] HP. Sombrerete, Enríquez 2558 (HUAZ).

Acourtia nana (A. Gray) Reveal \& R. M. King [=Perezia nana A. Gray]

HP. General Pánfilo Natera, Balleza 7725 (HUAZ); Mazapil, Balleza 6683 (HUAZ); Pinos, Balleza 5313 (HUAZ); Villa de Cos, Balleza 10638 (HUAZ).

${ }^{*}$ Acourtia parryi (A. Gray) Reveal \& R. M. King [=Perezia parryi A. Gray] HP. El Salvador, Balleza 9702 (HUAZ); Fresnillo; Balleza 5751 (HUAZ); Mazapil, Balleza 10677 (HUAZ); Miguel Auza, Balleza 5852 (HUAZ); Pánuco, Balleza 7661 (HUAZ); Río Grande, Balleza 10748 (HUAZ); Villa de Cos, Balleza 10646 (HUAZ).

*Acourtia platyphylla (A. Gray) Reveal \& R. M. King

HP. Río Grande, García 1191 (CHAPA).

Acourtia thurberi (A. Gray) Reveal \& R. M. King

HP. Genaro Codina, Balleza 6571 (HUAZ). 
Anexo. Continuación.

${ }^{*}$ Acourtia turbinata (La Llave) Reveal \& R. M. King [=Perezia arachnolepis B. L. Rob.] HP. Tlaltenango de Sánchez Román, Balleza 7963 (HUAZ).

${ }^{*}$ Acourtia wislizeni (A. Gray) Reveal \& R. M. King var. megacephala (A. Gray) Reveal \& R. M. King [=Perezia wislizeni A. Gray var. megacephala A. Gray]

HP. Juchipila, Balleza 9953 (HUAZ); Villanueva, Balleza 3303 (HUAZ).

Acourtia wrightii (A. Gray) Reveal \& R. M. King

HP. Mazapil, Balleza 9805 (HUAZ); Río Grande, Balleza 5700 (HUAZ); Villa de Cos, Balleza 6848 (HUAZ).

${ }^{*}$ Acourtia zacatecana B. L. Turner

HP. Cuauhtémoc, Del Castillo 23-ZA (CHAPA).

${ }^{*}$ Adenophyllum porophyllum (Cav.) Hemsl. var. cancellatum (Cass.) Strother [=Dyssodia porophyllum (Cav.) Cav. var. cancellata (Cass.) Strother]

HA. Jalpa, Balleza 6702 (HUAZ); Juan Aldama, Balleza 11306 (HUAZ); Juchipila, Balleza 10981 (HUAZ); Monte Escobedo, Balleza 7438 (HUAZ); Moyahua de Estrada, Enríquez 1715 (HUAZ); Nochistlán de Mejía, Balleza 5638 (HUAZ); Pinos; Balleza 11335 (HUAZ); Tabasco, Balleza 9481 (HUAZ); Tepechitlán, Balleza 3497 (HUAZ); Valparaíso, Balleza 9286 (HUAZ); Villanueva, Balleza 7240 (HUAZ).

${ }^{*}$ Adenophyllum porophyllum (Cav.) Hemsl. var. porophyllum [=Dyssodia porophyllum (Cav.) Cav. var. porophyllum (Cass.) Strother]

HA. Jerez, Enríquez 2162 (HUAZ).

Ageratina adenophora (Spreng.) R. M. King \& H. Rob. [=Eupatorium adenophorum Spreng.] HP. Juchipila; Balleza 8039 (HUAZ).

Ageratina areolaris (DC.) Gage [=Eupatorium areolare DC.]

AR. Juchipila, Balleza 11412 (HUAZ).

${ }^{*}$ Ageratina astillera (B. L. Turner) B. L. Turner

AR. Concepción del Oro, Balleza 6219 (HUAZ); Mazapil, Johnston 11568 (CHAPA).

${ }^{*}$ Ageratina blepharilepis (Sch. Bip.) R. M. King \& H. Rob. [=Eupatorium blepharilepis Sch. Bip.] HP. Valparaíso, McVaugh 25773 MEXU).

${ }^{*}$ Ageratina brevipes (DC.) R. M. King \& H. Rob. [=Eupatorium brevipes DC.]

AR. Fresnillo, Cronquist 10535 (MEXU); Guadalupe, Balleza 7708 (HUAZ); Juan Aldama, Bartholomew 3593 (MEXU); Juchipila, Balleza 7854 (HUAZ); Monte Escobedo, García 851 (MEXU); Pinos, Balleza 10172 (HUAZ); Río Grande, Wendt 2191 (CHAPA); Sain Alto, Sundberbg 2913 (MEXU); Tabasco, Balleza 9469 (HUAZ); Valparaíso, Balleza 7500 (HUAZ).

${ }^{*}$ Ageratina calaminthifolia (Kunth) R. M. King \& H. Rob. [=Eupatorium calaminthifolium Kunth] AR. Jerez, Balleza 6559 (HUAZ); Juchipila, Balleza 8208 (HUAZ); Tlaltenango de Sánchez Román, Balleza 6737 (HUAZ); Villa de Cos, Balleza 6860 (HUAZ). 
Anexo. Continuación.

*Ageratina calophylla (Greene) R. M. King \& H. Rob. [=Eupatorium calophyllum (Greene) B. L. Rob.] AR. Mazapil, Johnston 11568 (MEXU).

${ }^{*}$ Ageratina campyloclada (B. L. Rob.) R. M. King \& H. Rob. [=Eupatorium campylocladum B. L. Rob.] AR. Concepción del Oro, Lehto 21745 (MEXU).

${ }^{*}$ Ageratina choricephala (B. L. Rob.) R. M. King \& H. Rob. [=Eupatorium choricephalum B. L. Rob.] HP. Juchipila, Balleza 11552 (HUAZ); Moyahua de Estrada, Enríquez 1361 (HUAZ); Teúl de González Ortega; Barrie 1138 (MEXU); Tlaltenango de Sánchez Román, Rzedowski \& McVaugh 947 (MEXU).

${ }^{*}$ Ageratina espinosarum (A. Gray) R. M. King \& H. Rob. var. espinosarum [=Eupatorium espinosarum A. Gray var. espinosarum]

AR. Genaro Codina, Balleza 10368 (HUAZ); Guadalupe, Balleza 7709 (HUAZ); Pinos, Balleza 10174 (HUAZ); Valparaíso, McVaugh 25722 (MEXU); Zacatecas, Balleza 6997 (HUAZ).

*Ageratina espinosarum (A. Gray) R. M. King \& H. Rob. var. subintegrifolia (B. L. Rob.) B. L. Turner [=Eupatorium subintegrum (Greene) B. L. Rob.]

AR. Jerez, Balleza 7040 (HUAZ); Juchipila, Balleza 8862 (HUAZ); Mazapil, Balleza 9800 (HUAZ); Pánuco, Balleza 7657 (HUAZ); Sombrerete, Balleza 7117 (HUAZ); Villa González Ortega, Balleza 7757 (HUAZ).

*Ageratina jalpana B. L. Turner

HP. Jalpa, Breedlove 63992 (CAS, TEX).

*Ageratina leptodictyon (A. Gray) R. M. King \& H. Rob. [=Eupatorium leptodyction A. Gray]

HP. Juchipila, Balleza 8731 (HUAZ); Tlaltenango de Sánchez Román, Balleza 6733 (HUAZ).

Ageratina ligustrina (DC.) R. M. King \& H. Rob.

AR. Teúl de González Ortega, Balleza 8074 (HUAZ).

*Ageratina malacolepis (B. L. Rob.) R. M. King \& H. Rob. [=Eupatorium malacolepis B. L. Rob.] AR. Nochistlán de Mejía, Balleza 5589 (HUAZ).

${ }^{*}$ Ageratina oligocephala (DC.) R. M. King \& H. Rob.

HP. Tlaltenango de Sánchez Román, Rzedowski \& McVaugh 947 (MEXU).

${ }^{*}$ Ageratina palmeri (A. Gray) Gage [=Piptothrix pubens A. Gray]

AR. Juchipila, Balleza 11422 (HUAZ); Valparaíso, Balleza 7535a (HUAZ).

${ }^{*}$ Ageratina petiolaris (Moç. \& Sessé ex DC.) R. M. King \& H. Rob. [=Eupatorium petiolare Moç. \& Sess, ex DC.]

AR. Concepción del Oro, Balleza 5507 (HUAZ); Genaro Codina, Balleza 4246 (HUAZ); Jerez, Balleza 5389 (HUAZ); Jiménez del Teúl, Balleza 8198 (HUAZ); Nochistlán de Mejía, Balleza 5585 (HUAZ); Pinos, Balleza 10171 (HUAZ); Tlaltenango de Sánchez Román, Balleza 5248 (HUAZ); Villa González Ortega, Balleza 10569 (HUAZ); Zacatecas, Balleza 5270 (HUAZ).

Ageratina pichinchensis (Kunth) R. M. King \& H. Rob.

HP. Nochistlán de Mejía, Balleza 7594 (HUAZ). 
Anexo. Continuación.

${ }^{*}$ Ageratina rubricaulis (Kunth) R. M. King \& H. Rob. [=Eupatorium rubricaule Kunth] HP. Jalpa, Balleza 8974 (HUAZ).

${ }^{*}$ Ageratina scorodonioides (A. Gray) R. M. King \& H. Rob. [=Eupatorium scorodonioides A. Gray] AR. Chalchihuites, Balleza 7160 (HUAZ); Concepción del Oro, Balleza 6207 (HUAZ); Guadalupe, Balleza 7707 (HUAZ); Pinos, Balleza 6418 (HUAZ); Villa Hidalgo, Balleza 10598 (HUAZ); Zacatecas, Martínez 75 (MEXU).

${ }^{*}$ Ageratina triniona (McVaugh) R. M. King \& H. Rob. [=Eupatorium trinionum McVaugh] HP. Juchipila, Balleza 11396 (HUAZ); Monte Escobedo, Balleza 7478 (HUAZ); Nochistlán de Mejía, Balleza 7601 (HUAZ); Valparaíso, Balleza 9281 (HUAZ); Villanueva, Balleza 3447 (HUAZ).

${ }^{*}$ Ageratina venulosa (A. Gray) R. M. King \& H. Rob.

HP. Monte Escobedo, Narvaes 35 (CHAPA).

Ageratina wrightii (A. Gray) R. M. King \& H. Rob. [=Eupatorium wrightii A. Gray]

AR. Valparaíso, McVaugh 25733 (MEXU).

Ageratum corymbosum Zuccagni ex Pers. [=Ageratum corymbosum Zuccagni ex Pers. f. euryphyllum (B. L. Rob.) M. F. Johnson; =Ageratum corymbosum Zuccagni ex Pers. f. lactiflorum B. L. Rob]

HP. Benito Juárez, Balleza 7358 (HUAZ); Chalchihuites, Balleza 7187 (HUAZ); Concepción del Oro, Balleza 6623 (HUAZ); Genaro Codina, Balleza 10364 (HUAZ); Guadalupe, Balleza 7720 (HUAZ); Jalpa, Balleza 6700 (HUAZ); Jerez, Balleza 7033b (HUAZ); Jiménez del Teúl, Balleza 6135 (HUAZ); Juchipila, Balleza 7883 (HUAZ); Miguel Auza, Balleza 6894 (HUAZ); Monte Escobedo, Balleza 3875 (HUAZ); Moyahua de Estrada, Enríquez 1363 (HUAZ); Nochistlán de Mejía, Balleza 7586 (HUAZ); Pinos, Balleza 6419 (HUAZ); Sombrerete, Balleza 6178 (HUAZ); Tabasco, Balleza 9466 (HUAZ); Teúl de González Ortega, Balleza 6394 (HUAZ); Tlaltenango de Sánchez Román, Balleza 6750 (HUAZ); Valparaíso, Balleza 9267 (HUAZ); Villa de Cos, Balleza 6821 (HUAZ); Villa González Ortega, Balleza 6602 (HUAZ); Villanueva, Balleza 6501 (HUAZ); Zacatecas, Balleza 6998 (HUAZ).

${ }^{*}$ Alloispermum palmeri (S. Watson ex A. Gray) C. F. Fernández \& Urbatsch var. palmeri [=Calea palmeri A. Gray in S. Watson]

HP. Juchipila, Balleza 8603 (HUAZ); Teúl de González Ortega, Balleza 6403 (HUAZ).

Alloispermum scabrum (Lag.) H. Rob. [=Calea scabra (Lag.) B. L. Rob.]

HP. Jalpa, Balleza 8971 (HUAZ); Nochistlán de Mejía, Balleza 7597 (HUAZ); Teúl de González Ortega, Balleza 4131 (HUAZ); Valparaíso, Balleza 9329 (HUAZ).

Ambrosia artemisiifolia L.

HP. Calera, Aguilar 29 (CHAPA); Noria de Ángeles, Balleza 7770 (HUAZ); Río Grande, Balleza 5709 (HUAZ).

${ }^{*}$ Ambrosia canescens A. Gray

HP. Loreto, Balleza 6588 (HUAZ); Mazapil, Balleza 11250 (HUAZ); Pinos, Balleza 6427 (HUAZ);

Sombrerete, Balleza 7203 (HUAZ). 
Anexo. Continuación.

Ambrosia confertiflora DC.

HP. Calera, Scora 2622 (MEXU); Fresnillo, Balleza 5969 (HUAZ); Loreto, Balleza 6587 (HUAZ); Mazapil, Balleza 11043 (HUAZ); Monte Escobedo, Balleza 7456 (HUAZ); Morelos, Delgado 1160GD (MEXU); Pánuco, Balleza 7650 (HUAZ); Pinos, Balleza 11325 (HUAZ); Río Grande, Balleza 10722 (HUAZ); Sombrerete, Tenorio 2171 (MEXU); Villa de Cos, Balleza 10653 (HUAZ); Villa González Ortega, Balleza 6603 (HUAZ); Zacatecas, Balleza 62 (HUAZ).

Ambrosia psilostachya DC. var. psilostachya HP. Mazapil, Balleza 6639 (HUAZ); Miguel Auza, Balleza 6892 (HUAZ); Monte Escobedo, Balleza 7431 (HUAZ); Pinos, Balleza 6429 (HUAZ); Sombrerete, Balleza 6151 (HUAZ); Valparaíso, Balleza 6097 (HUAZ).

Aphanostephus ramosissimus (DC.) A. Gray var. humilis (Benth.) B. L. Turner \& Birdsong HP. Guadalupe, Villaseñor 544 (MEXU).

*Aphanostephus ramosissimus (DC.) A. Gray var. ramosus (DC.) B. L. Turner \& Birdsong HP. Chalchihuites, Balleza 6082 (HUAZ); Cuauhtémoc, Balleza 10400 (HUAZ); Fresnillo, Balleza 11190 (HUAZ); General Francisco Murguía, Balleza 5900 (HUAZ); Guadalupe, Balleza 7005 (HUAZ); Juan Aldama, Balleza 5818 (HUAZ); Mazapil, Balleza 10663 (HUAZ); Miguel Auza, Balleza 5854 (HUAZ); Nochistlán de Mejía, Balleza 5613 (HUAZ); Noria de Ángeles, Balleza 10589 (HUAZ); Pinos, Balleza 11330 (HUAZ); Río Grande, Balleza 5683 (HUAZ); Sombrerete, Balleza 5771 (HUAZ); Valparaíso, Balleza 6087 (HUAZ); Villa de Cos, Balleza 10331 (HUAZ); Villa González Ortega, Balleza 10583 (HUAZ).

Archibaccharis serratifolia (Kunth) S. F. Blake HP. Jalpa, Balleza 8976 (HUAZ); Juchipila, Balleza 9546 (HUAZ); Tlaltenango de Sánchez Román, Balleza 6768 (HUAZ).

Artemisia dracunculus L. var. glauca (Pall.) Besser HP. Mazapil, Balleza 11051 (HUAZ).

*Artemisia klotzschiana Besser HA. Concepción del Oro, Gonzáles s/n (CHAPA); Mazapil, Balleza 9778 (HUAZ); Pinos, Balleza 11324 (HUAZ); Villa González Ortega, Balleza 7755 (HUAZ).

Artemisia ludoviciana Nutt. [=Artemisia ludoviciana Nutt. subsp. mexicana (Spreng.) Keck] HP. Benito Juárez, Balleza 7379 (HUAZ); Chalchihuites, Balleza 7171 (HUAZ); Concepción del Oro, Balleza 6635 (HUAZ); Fresnillo, Balleza 11189 (HUAZ); Genaro Codina, Balleza 10354 (HUAZ); Guadalupe, Balleza 7010 (HUAZ); Jalpa, Balleza 7969 (HUAZ); Jerez, Balleza 6520 (HUAZ); Juan Aldama, Balleza 11156 (HUAZ); Juchipila, Balleza 11437 (HUAZ); Mazapil, Balleza 11054 (HUAZ); Miguel Auza, Balleza 6867, (HUAZ); Monte Escobedo, Balleza 7418 (HUAZ); Nochistlán de Mejía, Balleza 9013 (HUAZ); Noria de Ángeles, Balleza 10590 (HUAZ); Pánuco, Balleza 7639 (HUAZ); Pinos, Balleza 10160 (HUAZ); Río Grande, Balleza 10737 (HUAZ); Sombrerete, Balleza 7206 (HUAZ); Tabasco, Balleza 9463 (HUAZ); Tlaltenango de Sánchez Román, Balleza 6709 (HUAZ); Valparaíso, Rzedowski 17542 (MEXU); Villa de Cos, Balleza 10650 (HUAZ); Villanueva, Balleza 7246 (HUAZ). 
Anexo. Continuación.

Aster moranensis Kunth var. moranensis

HP. Monte Escobedo, Balleza 8082 (HUAZ).

Aster pauciflorus Nutt.

HA. Miguel Auza, Balleza 6887 (HUAZ); Pinos, Balleza 6422 (HUAZ).

*Aster potosinus A. Gray

HP. Fresnillo, Balleza 6016 (HUAZ); Juchipila, Balleza 7859 (HUAZ).

Aster subulatus Michx. var. subulatus

HA. Benito Juárez, Balleza 7359 (HUAZ); General Francisco Murguía, Balleza 6955 (HUAZ); Jalpa, Balleza 8661 (HUAZ); Jerez, Balleza 6515 (HUAZ); Juan Aldama, Balleza 11159 (HUAZ); Juchipila, Balleza 9092 (HUAZ), Loreto, Balleza 6586 (HUAZ); Miguel Auza, Balleza 6875 (HUAZ); Moyahua de Estrada, Enríquez 1569 (HUAZ); Pinos, Balleza 6411 (HUAZ); Sombrerete, Balleza 7138 (HUAZ); Valparaíso, Balleza 7516 (HUAZ); Villa González Ortega, Balleza 10579 (HUAZ); Zacatecas, Balleza 4351 (HUAZ).

${ }^{*}$ Astranthium orthopodum (B. L. Rob. \& Fernald) Larsen HP. Chalchihuites, Balleza 6109 (HUAZ); Sombrerete, García 871 (CHAPA); Sombrerete, García 871 (MEXU); Zacatecas, Balleza 77 (HUAZ).

${ }^{*}$ Aztecaster matudae (Rzed.) G. L. Nesom

AR. Chalchihuites, Balleza 8193 (HUAZ); Mazapil, Balleza 10678 (HUAZ); Río Grande, Balleza 10760 (HUAZ).

Baccharis heterophylla Kunth

AR. Jalpa, Balleza 8392 (HUAZ); Jerez, Balleza 5410 (HUAZ); Juchipila, Balleza 8363 (HUAZ); Monte Escobedo, Balleza 5550 (HUAZ); Nochistlán de Mejía, Balleza 5587 (HUAZ); Valparaíso, Balleza 8432 (HUAZ).

Baccharis neglecta Britton

AR. Chalchihuites, Balleza 7152 (HUAZ); General Francisco Murguía, Balleza 6960 (HUAZ); Miguel Auza, Balleza 6865 (HUAZ); Sombrerete, Balleza 7132 (HUAZ).

${ }^{*}$ Baccharis occidentalis S. F. Blake

HP. Sombrerete, Balleza 7080 (HUAZ).

${ }^{*}$ Baccharis palmeri Greenm.

HP. Monte Escobedo, Balleza 7426 (HUAZ); Villanueva, Balleza 7233 (HUAZ).

Baccharis pteronioides DC.

AR. Chalchihuites, Balleza 6116 (HUAZ); El Salvador, Balleza 8298 (HUAZ); Fresnillo, Gonzáles 337 (MEXU); Genaro Codina, Balleza 10353 (HUAZ); General Joaquín Amaro, Vela 3096 (MEXU); Jalpa, Balleza 5572 (HUAZ); Jerez, Balleza 5377 (HUAZ); Juan Aldama, Balleza 5826 (HUAZ); Juchipila, Balleza 8264 (HUAZ); Mazapil, Balleza 5525 (HUAZ); Miguel Auza, Balleza 5857 (HUAZ); Monte Escobedo, Balleza 5530 (HUAZ); Nochistlán de Mejía, Balleza 5621 (HUAZ); Pinos, Balleza 8230 (HUAZ); Río Grande, Balleza 5680 (HUAZ); Sombrerete, Balleza 5784 (HUAZ); Tabasco, Balleza 8278 (HUAZ); Tlaltenango de Sánchez Román, Balleza 8523 (HUAZ); Villa de Cos, Balleza 5444 (HUAZ); Villa Hidalgo, Balleza 5374 (HUAZ); Villanueva, McVaugh 11983 (MEXU). 
Anexo. Continuación.

Baccharis salicifolia (Ruiz \& Pav.) Pers.

AR. Fresnillo, Balleza 5984 (HUAZ); Genaro Codina, Balleza 10350 (HUAZ); General Joaquín Amaro, Balleza 5948 (HUAZ); Guadalupe, Balleza s/n (HUAZ); Jalpa, Balleza 5574 (HUAZ); Jerez, Balleza 6521 (HUAZ); Juan Aldama, Balleza 11308 (HUAZ); Mazapil, Balleza 11143 (HUAZ); Monte Escobedo, Balleza 7458 (HUAZ); Pinos, Balleza 6432 (HUAZ); Río Grande, Balleza 10738 (HUAZ); Sombrerete, Balleza 6176 (HUAZ); Tepechitlán, Balleza 6298 (HUAZ); Teúl de González Ortega, Balleza 7315 (HUAZ); Valparaíso, Balleza 6031 (HUAZ); Villa González Ortega, Balleza 10575 (HUAZ); Villanueva, Balleza 5920 (HUAZ); Zacatecas, Balleza 6992 (HUAZ).

Baccharis serrifolia DC.

AR. Jerez, Balleza 7045 (HUAZ).

${ }^{*}$ Baccharis squarrosa Kunth

HP. Jalpa, Balleza 8391 (HUAZ); Juchipila, Balleza 10022 (HUAZ); Tlaltenango de Sánchez Román, Balleza 7961 (HUAZ).

${ }^{*}$ Baccharis sulcata DC.

HP. Concepción del Oro, Balleza 6205 (HUAZ); Villa García, Martínez s/n (MEXU).

Baccharis thesioides Kunth

AR. Genaro Codina, Balleza 6566 (HUAZ); Jalpa, Balleza 7552 (HUAZ); Juchipila, Panero 6856 (MEXU); Nochistlán de Mejía, Balleza 7571 (HUAZ); Teúl de González Ortega, Balleza 7314 (HUAZ); Valparaíso, Balleza 7522 (HUAZ); Zacatecas, Balleza 217 (HUAZ).

Bahia absinthifolia Benth. var. absinthifolia

HP. Concepción del Oro, Balleza 3984 (HUAZ); Cuauhtémoc, Balleza 10394 (HUAZ); El Salvador, Balleza 9710 (HUAZ); Fresnillo, Balleza 7229 (HUAZ); General Francisco Murguía, Balleza 6939 (HUAZ); General Pánfilo Natera, Balleza 7736 (HUAZ); Mazapil, Balleza 6234 (HUAZ); Miguel Auza, Balleza 5845 (HUAZ); Noria de Ángeles, Balleza 6597 (HUAZ); Pánuco, Balleza 7651 (HUAZ); Pinos, Balleza 8959 (HUAZ); Río Grande, Balleza 5699 (HUAZ); Villa de Cos, Balleza 7697 (HUAZ); Zacatecas, Ellison 112 (MEXU).

*Bahia glandulosa Greenm.

HA. Benito Juárez, Balleza 7356 (HUAZ); Chalchihuites, Balleza 7161 (HUAZ); Jalpa, Balleza 8993 (HUAZ); Jerez, Balleza 6512 (HUAZ); Miguel Auza, Balleza 6904 (HUAZ); Monte Escobedo, Balleza 7429 (HUAZ); Nochistlán de Mejía, Balleza 9020 (HUAZ); Noria de Ángeles, Balleza 7768 (HUAZ); Pinos, Balleza 11332 (HUAZ); Sombrerete, Balleza 7096 (HUAZ); Teúl de González Ortega, Balleza 6309 (HUAZ); Valparaíso, Balleza 9369 (HUAZ); Villanueva, Balleza 7255 (HUAZ); Zacatecas, Balleza 658 (HUAZ).

${ }^{*}$ Bahia schaffneri S. Watson var. schaffneri

HA. El Salvador, Balleza 9686 (HUAZ); Fresnillo, Balleza 11188 (HUAZ); General Pánfilo Natera, Balleza 4358 (HUAZ); Guadalupe, García 803 (MEXU); Juan Aldama, Balleza 11158 (HUAZ); Mazapil, Balleza 9741 (HUAZ); Noria de Ángeles, Balleza 7769 (HUAZ); Pánuco, Balleza 7653 (HUAZ); Pinos, Balleza 8956 (HUAZ); Río Grande, Balleza 10702 (HUAZ); Villa de Cos, Balleza 6784 (HUAZ); Villa González Ortega; Balleza 7759 (HUAZ); Villanueva, Breedlove 61559 (MEXU); Zacatecas; Balleza 231 (HUAZ). 
Anexo. Continuación.

Baileya multiradiata Harv. \& A. Gray ex Torr. [=Baileya australis Rydb.]

HP. Fresnillo, Balleza 5679 (HUAZ); Guadalupe, Hernández 103006x (CHAPA).

Barkleyanthus salicifolius (Kunth) H. Rob. \& Brettell [=Senecio salignus DC.]

AR. Chalchihuites, Balleza 8196 (HUAZ); Concepción del Oro, Balleza 8017 (HUAZ); Fresnillo, Balleza 5739 (HUAZ); General Joaquín Amaro, Balleza 5339 (HUAZ); Guadalupe, Balleza 5271 (HUAZ); Jerez, Balleza 5376 (HUAZ); Juan Aldama, Balleza 5822 (HUAZ); Juchipila, Balleza 8153 (HUAZ); Loreto, Balleza 8118 (HUAZ); Luis Moya, Balleza 8116 (HUAZ); Mazapil, Balleza 5510 (HUAZ); Miguel Auza, Balleza 8105 (HUAZ); Monte Escobedo, Balleza 8084 (HUAZ); Nochistlán de Mejía, Balleza 5596 (HUAZ); Noria de Ángeles, Balleza 5294 (HUAZ); Pinos, Balleza 5312 (HUAZ); Río Grande, Balleza 8098 (HUAZ); Sombrerete, Balleza 8173 (HUAZ); Teúl de González Ortega, Balleza 8072 (HUAZ); Tlaltenango de Sánchez Román, Balleza 5253 (HUAZ); Valparaíso, Balleza 8437 (HUAZ); Villa de Cos, Balleza 4180 (HUAZ); Villa González Ortega, Balleza 8123 (HUAZ); Villanueva, McVaugh 11985 (MEXU); Zacatecas, Balleza 366 (HUAZ).

Berlandiera lyrata Benth. var. lyrata

HP. Chalchihuites, Balleza 6115 (HUAZ); Cuauhtémoc, Balleza 6578 (HUAZ); Guadalupe, Balleza 10336 (HUAZ); Jiménez del Teúl, Balleza 6140 (HUAZ); Miguel Auza, Balleza 5867 (HUAZ); Sombrerete, Balleza 6152 (HUAZ).

${ }^{*}$ Bidens acrifolia Sherff var. acrifolia

HP. Valparaíso, Balleza 9426 (HUAZ).

${ }^{*}$ Bidens angustissima Kunth var. angustissima

HP. Jerez, Balleza 6564 (HUAZ); Juchipila, Balleza 11449 (HUAZ); Tlaltenango de Sánchez Román, Balleza 8531 (HUAZ); Valparaíso, Balleza 9381 (HUAZ).

Bidens aurea (Aiton) Sherff

HP. Benito Juárez, Balleza 6340 (HUAZ); Jerez, Balleza 6554 (HUAZ); Miguel Auza, Balleza 6907 (HUAZ); Tlaltenango de Sánchez Román, Balleza 6757 (HUAZ); Valparaíso, Balleza 9430 (HUAZ).

${ }^{*}$ Bidens bigelovii A. Gray var. angustiloba (DC.) Ballard

HA. Juchipila, Balleza 9235 (HUAZ); Moyahua de Estrada, Balleza 7261b (HUAZ).

Bidens ferulifolia (Jacq.) DC.

HA. Benito Juárez, Balleza 6321 (HUAZ); Cuauhtémoc, Balleza 10388 (HUAZ); Guadalupe, Balleza 7012 (HUAZ); Jalpa, Balleza 8984 (HUAZ); Jerez, Balleza 6538 (HUAZ); Juan Aldama, Balleza 11183 (HUAZ); Juchipila, Balleza 9942 (HUAZ); Miguel Auza, Balleza 5874 (HUAZ); Monte Escobedo, Balleza 7447 (HUAZ); Nochistlán de Mejía, Balleza 7577 (HUAZ); Pinos, Balleza 6447 (HUAZ); Sombrerete, Balleza 7223 (HUAZ); Teúl de González Ortega, Balleza 6308 (HUAZ); Tlaltenango de Sánchez Román, Rzedowski \& McVaugh 1051 (MEXU); Villanueva, Balleza 7249 (HUAZ).

Bidens lemmonii A. Gray

HA. Apulco, Balleza 5643 (HUAZ); Nochistlán de Mejía, Balleza 9026 (HUAZ); Tlaltenango de Sánchez Román, Rzedowski \& McVaugh 908 (MEXU).

Bidens odorata Cav. var. odorata

HA. Chalchihuites, Balleza 7154 (HUAZ); Concepción del Oro, Balleza 6630 (HUAZ); Cuauhtémoc, Balleza 10408 (HUAZ); El Salvador, Balleza 9711 (HUAZ); Fresnillo, Balleza 5664 (HUAZ); General 
Anexo. Continuación.

Joaquín Amaro, Balleza 9532 (HUAZ); Jalpa, Balleza 8987 (HUAZ); Jerez, Balleza 5398 (HUAZ); Juan Aldama, Balleza 5824 (HUAZ); Juchipila, Balleza 7787 (HUAZ); Mazapil, Balleza 9777 (HUAZ); Miguel Auza, Balleza 6917 (HUAZ); Monte Escobedo, Balleza 7434 (HUAZ); Moyahua de Estrada, Enríquez 1112 (HUAZ); Noria de Ángeles, Balleza 7767 (HUAZ); Pánuco; Balleza 7640 (HUAZ); Pinos, Balleza 6423 (HUAZ); Río Grande, Balleza 10759 (HUAZ); Sombrerete, Balleza 7215 (HUAZ); Tepechitlán, Balleza 3482 (HUAZ); Valparaíso, Balleza 8454 (HUAZ); Villa de Cos, Balleza 10654 (HUAZ); Villa González Ortega, Balleza 7744 (HUAZ); Zacatecas, Balleza 660 (HUAZ).

Bidens pilosa L. var. pilosa

HA. Valparaiso, Rose 2426 (Citado en McVaugh, 1984).

Bidens rostrata Melchert

HA. Juchipila, Balleza 9874 (HUAZ).

*Bidens schaffneri (A. Gray) Sherff var. schaffneri

HP. Miguel Auza, Balleza 6870 (HUAZ).

${ }^{*}$ Bidens serrulata (Poir.) Desf. var. serrulata

HA. Valparaíso, Balleza 9444 (HUAZ).

Bidens triplinervia Kunth var. triplinervia

HA. Valparaíso, Balleza 9442 (HUAZ).

*Bolanosa coulteri A. Gray

HP. Juchipila, Balleza 11444 (HUAZ); Moyahua de Estrada, Enríquez 424 (MEXU).

*Brickellia adenolepis (B. L. Rob.) Shinners

HP. Tlaltenango de Sánchez Román, McVaugh 25645 (Citado en McVaugh, 1984).

*Brickellia cardiophylla B. L. Rob.

HP. Juchipila, Balleza 7979b (HUAZ).

${ }^{*}$ Brickellia coulteri A. Gray var. adenopoda (B. L. Rob.) B. L. Turner

AR. Moyahua de Estrada, Enríquez 1705 (HUAZ); Río Grande, Balleza 5715 (HUAZ).

Brickellia coulteri A. Gray var. brachiata (A. Gray) B. L. Turner

AR. Moyahua de Estrada, Enríquez 1484 (HUAZ).

Brickellia diffusa (Vahl) A. Gray

HA. Moyahua de Estrada, Enríquez 1297 (HUAZ).

Brickellia eupatorioides (L.) Shinners var. chlorolepis (Wooton \& Standl.) B. L. Turner [=Brickellia corymbosa (DC.) A. Gray]

HA. Chalchihuites, Balleza 7179 (HUAZ); Guadalupe, García 806 (CHAPA); Juan Aldama, Balleza 5840 (HUAZ); Mazapil, Balleza 8334 (HUAZ); Miguel Auza, Balleza 6916 (HUAZ); Nochistlán de Mejía, Balleza 5627 (HUAZ); Río Grande, Balleza 5681 (HUAZ); Sombrerete, Balleza 7217 (HUAZ); Villa de Cos, Balleza 6199 (HUAZ); Villa González Ortega, Balleza 10568 (HUAZ); Zacatecas, Balleza 701 (HUAZ). 
Anexo. Continuación.

${ }^{*}$ Brickellia jaliscensis McVaugh

HP. Tlaltenango de Sánchez Román, McVaugh 25629 (Citado en McVaugh, 1984).

Brickellia laciniata A. Gray

AR. Río Grande, Balleza 5714 (HUAZ).

*Brickellia lanata (DC.) A. Gray

HP. García de la Cadena, Balleza 7299 (HUAZ); Jalpa, Balleza 7551 (HUAZ); Juchipila, Balleza 7864

(HUAZ); Monte Escobedo, García 846 (CHAPA).

${ }^{*}$ Brickellia laxiflora (Brandegee) B. L. Turner

HA. Moyahua de Estrada, Enríquez 754 (HUAZ).

Brickellia lemmonii A. Gray var. conduplicata (B. L. Rob.) B. L. Turner

AR. Apulco, Balleza 5644 (HUAZ); Sombrerete, Balleza 6196 (HUAZ).

${ }^{*}$ Brickellia monocephala B. L. Rob.

HP. Jalpa, Balleza 6456 (HUAZ); Teúl de González Ortega, Balleza 6392 (HUAZ).

Brickellia oliganthes (Less.) A. Gray

HP. Juchipila, Balleza 7841 (HUAZ); Teúl de González Ortega, Balleza 8069a (HUAZ); Tlaltenango de Sánchez Román, McVaugh 25654 (HUAZ).

${ }^{*}$ Brickellia palmeri A. Gray

AR. Juan Aldama, Bartholomew 3602 (MEXU); Valparaíso, Balleza 7531 (HUAZ); Zacatecas, Pringle 4054 (MEXU).

${ }^{*}$ Brickellia pedunculosa (DC.) R. M. King \& H. Rob.

HP. Tlaltenango de Sánchez Román, McVaugh 25606 (Citado en McVaugh, 1984).

${ }^{*}$ Brickellia robinsoniana S. F. Blake

HP. Río Grande, Balleza 10730 (HUAZ).

Brickellia scoparia (DC.) A. Gray var. scoparia

HP. García de la Cadena, Cronquist 9827 (MEXU); General Joaquín Amaro, Balleza 9517 (HUAZ);

Sombrerete, Tenorio 2189 (MEXU); Villanueva, Balleza 3603 (HUAZ).

${ }^{*}$ Brickellia secundiflora (Lag.) A. Gray var. nepetifolia (Kunth) B. L. Rob.

AR. Juchipila, Balleza 7882 (HUAZ); Monte Escobedo, Barrie 1131 (MEXU).

${ }^{*}$ Brickellia secundiflora (Lag.) A. Gray var. secundiflora

AR. Chalchihuites, Balleza 7156 (HUAZ); Jalpa, Balleza 5569 (HUAZ); Juan Aldama, Balleza 5828 (HUAZ); Juchipila, Balleza 7997 (HUAZ); Monte Escobedo, Balleza 5552 (HUAZ); Nochistlán de Mejía, Balleza 5590 (HUAZ); Sombrerete, Balleza 7128 (HUAZ); Teúl de González Ortega, Balleza 8054 (HUAZ); Villa González Ortega, Balleza 7739 (HUAZ).

${ }^{*}$ Brickellia spinulosa (A. Gray) A. Gray

AR. Chalchihuites, Rzedowski 8756 (MEXU); Guadalupe, Gonzáles s/n (MEXU); Juan Aldama, Balleza 5830 (HUAZ); Sombrerete, Miranda 8695b (MEXU). 
Anexo. Continuación.

${ }^{*}$ Brickellia subuligera (Schauer) B. L. Turner [=Barroetea subuligera (Schauer) A. Gray] HA. General Francisco Murguía, Balleza 6957 (HUAZ); Jiménez del Teúl, Balleza 7194 (HUAZ); Juchipila, Balleza 8047 (HUAZ); Moyahua de Estrada, Enríquez 1673 (HUAZ); Tabasco, Balleza 9536 (HUAZ); Villa de Cos, Balleza 6837 (HUAZ).

${ }^{*}$ Brickellia vernicosa B. L. Rob.

Coleosanthus vernicosus (B. L. Rob.) S. F. Blake

AR. Genaro Codina, Balleza 4247 (HUAZ); Sombrerete, Balleza 8174 (HUAZ).

Brickellia veronicifolia (Kunth) A. Gray [=Brickellia veronicifolia var. petrophila (B. L. Rob.) B. L. Rob.] AR. Chalchihuites, Dorado 1601 (MEXU); Concepción del Oro, Balleza 3994 (HUAZ); Fresnillo, Balleza 6000 (HUAZ); Genaro Codina, Balleza 10351 (HUAZ); General Francisco Murguía, Balleza 5901 (HUAZ); Guadalupe, Balleza 7710 (HUAZ); Jalpa, Balleza 8000 (HUAZ); Jerez, Balleza 5385 (HUAZ); Juan Aldama, Balleza 11173 (HUAZ); Juchipila, Balleza 7958 (HUAZ); Mazapil, Balleza 9804 (HUAZ); Noria de Ángeles, Balleza 5297 (HUAZ); Pánuco, Balleza 7641 (HUAZ); Pinos, Balleza 5333 (HUAZ); Río Grande, García 1193 (MEXU); Valparaíso, Balleza 6091 (HUAZ); Villa de Cos, Balleza 6859 (HUAZ); Villa García, Martínez s/n (MEXU); Villa González Ortega, Balleza 7761 (HUAZ); Zacatecas, Balleza s/n (HUAZ).

Calea ternifolia Kunth var. ternifolia [=Calea zacatechichi Schldl.]

AR. García de la Cadena, Balleza 7308 (HUAZ); Juchipila, Balleza 7818 (HUAZ).

Calea urticifolia (Mill.) DC. var. urticifolia

AR. Juchipila, Balleza 9955 (HUAZ); Monte Escobedo, García 52 (MEXU).

Calyptocarpus vialis Less.

HP. General Francisco Murguía, Balleza 6951 (HUAZ); Mazapil; Balleza 11263 (HUAZ).

Carminatia tenuiflora DC.

HA. Jalpa, Balleza 7558 (HUAZ); Juchipila, Balleza 9636 (HUAZ); Monte Escobedo, Balleza 7454 (HUAZ); Moyahua de Estrada, Enríquez 1301 (HUAZ); Teúl de González Ortega, Balleza 7354 (HUAZ); Villanueva, Balleza 3451 (HUAZ).

${ }^{*}$ Carphochaete grahamii A. Gray [=Carphochaete gummifera McVaugh]

HP. Teúl de González Ortega, McVaugh 25617 (Citado en McVaugh, 1984); Sombrerete, Balleza 7084 (HUAZ); Valparaíso, McVaugh 25772 (MEXU).

Centaurea rothrockii Greenm.

HA. Benito Juárez, Balleza 7392 (HUAZ); Valparaíso, Balleza 9333 (HUAZ).

Chaetopappa bellioides (A. Gray) Shinners

HP. Cañitas de Felipe Pescador, Balleza 11199 (HUAZ); Chalchihuites, Balleza 8191 (HUAZ); Fresnillo, Balleza 6002 (HUAZ); Jerez, Balleza 5388 (HUAZ); Jiménez del Teúl, Balleza 6138 (HUAZ); Juan Aldama, Balleza 5835 (HUAZ); Loreto, Balleza 5285 (HUAZ); Mazapil, Balleza 9784 (HUAZ); Pinos, Balleza 8955 (HUAZ); Villa de Cos, Balleza 7696 (HUAZ); Villanueva, Balleza 3413 (HUAZ). 
Anexo. Continuación.

Chaetopappa ericoides (Torr.) G. L. Nesom

HP. Cuauhtémoc, Balleza 10404 (HUAZ); Jerez, Balleza 5387 (HUAZ); Loreto, Banda s/n (CHAPA); Mazapil, Balleza 6228 (HUAZ); Pinos, Balleza 8239 (HUAZ); Río Grande, Balleza 5698 (HUAZ); Sombrerete, Balleza 8186 (HUAZ); Villa de Cos, Balleza 10694 (HUAZ).

Chaptalia runcinata Kunth

HA. Valparaíso, Balleza 9435 (HUAZ).

Chloracantha spinosa (Benth.) G. L. Nesom var. spinosa [=Aster spinosus Benth.]

AR. Fresnillo; Balleza 6015 (HUAZ); General Francisco Murguía, Balleza 5895 (HUAZ); Miguel Auza, Balleza 6880 (HUAZ); Sombrerete, Balleza 6198 (HUAZ); Valparaíso, Balleza 6081 (HUAZ).

Chromolaena collina (DC.) R. M. King \& H. Rob. [=Eupatorium collinum DC. var. mendezii (DC.) McVaugh]

AR. Juchipila, Balleza 7798 (HUAZ); Moyahua de Estrada, Enríquez 1681 (HUAZ); Tabasco, Balleza 9537 (HUAZ); Valparaíso, Balleza 7534 (HUAZ).

Chromolaena odorata (L.) R. M. King \& H. Rob. [=Eupatorium odoratum L.]

HP. Moyahua de Estrada, Enríquez 1706 (HUAZ).

${ }^{*}$ Chromolaena ovaliflora (Hook. \& Arn.) R. M. King \& H. Rob. [=Eupatorium ovaliflorum Hook. \& Arn.] AR. Juchipila, Balleza 7848 (HUAZ); Teúl de González Ortega, Balleza 8064 (HUAZ).

${ }^{*}$ Chromolaena pulchella (Kunth) R. M. King \& H. Rob. [=Eupatorium pulchellum Kunth in H.B.K.] HP. Tlaltenango de Sánchez Román, Rzedowski \& McVaugh 1008 (ENCB); Valparaíso, Feddema 2397 (ENCB).

Chrysactinia mexicana A. Gray

AR. Concepción del Oro, Balleza 6217 (HUAZ); Mazapil, Balleza 5520 (HUAZ); Pinos, Balleza 10243 (HUAZ); Villa de Cos, Balleza 5443 (HUAZ).

Chrysanthellum indicum DC. var. mexicanum (Greenm.) B. L. Turner

HA. Juchipila, Balleza 8911 (HUAZ).

${ }^{*}$ Cirsium acrolepis (Petrak) G. B. Ownbey

HP. Juchipila, Balleza 8165 (HUAZ).

${ }^{*}$ Cirsium anartiolepis Petrak

HP. Jalpa, Balleza 9034 (HUAZ); Juchipila, Balleza 8036 (HUAZ); Monte Escobedo, Balleza 7443 (HUAZ); Nochistlán de Mejía, Balleza 7590 (HUAZ); Valparaíso, Balleza 9418 (HUAZ).

Cirsium horridulum Michx.

HP. Tlaltenango de Sánchez Román, Balleza 8406 (HUAZ).

Cirsium mexicanum DC.

HP. Concepción del Oro, Balleza 6225 (HUAZ); Mazapil, Balleza 6292 (HUAZ); Sombrerete, Balleza 6153 (HUAZ); Villa de Cos, Balleza 6200 (HUAZ).

Cirsium ochrocentrum (A. Gray) A. Gray

HP. Miguel Auza, Balleza 6896 (HUAZ). 
Anexo. Continuación.

${ }^{*}$ Cirsium pringlei (S. Watson) Petrak

HP. Concepción del Oro, Marroquín 187 (CHAPA); Villa de Cos, Banda s/n (CHAPA).

${ }^{*}$ Cirsium rhaphilepis (Hemsl.) Petrak

HP. Mazapil, Díaz s/n (MEXU).

Conoclinium betonicifolium (Mill.) R. M. King \& H. Rob.

HP. Miguel Auza, Balleza 5873 (HUAZ).

Conoclinium dissectum A. Gray

HP. General Francisco Murguía, Balleza 5891a (HUAZ); Mazapil, Balleza 9752 (HUAZ); Villa de Cos, Balleza 6836 (HUAZ); Villa de Cos, Balleza 10620 (HUAZ).

Conyza bonariensis (L.) Cronquist

Fresnillo, Balleza 5975 (HUAZ); Jalpa, Balleza 6481 (HUAZ); HA. Nochistlán de Mejía, Balleza 5625 (HUAZ); Pinos, Balleza 6406 (HUAZ); Tabasco, Balleza 5957 (HUAZ); Tlaltenango de Sánchez Román, Balleza 8402 (HUAZ).

Conyza canadensis (L.) Cronquist var. glabrata (A. Gray) Cronquist

HA. Monte Escobedo, Balleza 7441 (HUAZ); Valparaíso, Balleza 6070 (HUAZ).

Conyza canadensis (L.) Cronquist var. pusilla (Nutt.) Cronquist

HA. Fresnillo, Balleza 5962 (HUAZ); Jerez, Balleza 6551 (HUAZ); Valparaíso, Balleza 6101 (HUAZ).

Conyza coronopifolia Kunth

HA. Chalchihuites, Balleza 6095b (HUAZ); Jalpa, Balleza 8660b (HUAZ); Juchipila, Balleza 8741 (HUAZ); Nochistlán de Mejía, Balleza 5605 (HUAZ); Pinos, Balleza 10186 (HUAZ); Sombrerete, Balleza 6156 (HUAZ); Valparaíso, Balleza 6052 (HUAZ).

${ }^{*}$ Conyza microcephala Hemsl.

HA. Chalchihuites, Balleza 7169 (HUAZ); García de la Cadena, Balleza 7290 (HUAZ); General Joaquín Amaro, Balleza 5945 (HUAZ); Jalpa, Balleza 8387 (HUAZ); Jerez, Balleza 7065 (HUAZ); Juchipila, Balleza 11622 (HUAZ); Monte Escobedo, Balleza 7432 (HUAZ); Teúl de González Ortega, Balleza 6305 (HUAZ); Tlaltenango de Sánchez Román, Balleza 8533 (HUAZ); Valparaíso, Balleza 7517 (HUAZ).

${ }^{*}$ Coreopsis cyclocarpa S. F. Blake var. cyclocarpa

HP. Benito Juárez, Balleza 7404b (HUAZ); Tlaltenango de Sánchez Román, Balleza 6758 (HUAZ).

${ }^{*}$ Coreopsis mcvaughii D. J. Crawford

HP. Sombrerete, Enríquez 2009 (HUAZ).

${ }^{*}$ Coreopsis rudis (Benth.) Hemsl.

AR. Sombrerete, Balleza 7090 (HUAZ); Tlaltenango de Sánchez Román, Rzedowski \& McVaugh 907 (CHAPA).

Cosmos bipinnatus Cav.

HA. Benito Juárez, Balleza 6345 (HUAZ); El Salvador, Balleza 9714 (HUAZ); García de la Cadena, Balleza 7305 (HUAZ); General Joaquín Amaro, Balleza 9516 (HUAZ); Jalpa, Balleza 8992 (HUAZ); 
Anexo. Continuación.

Jerez, Balleza 6541 (HUAZ); Juchipila, Balleza 7894 (HUAZ); Monte Escobedo, Balleza 7442 (HUAZ); Nochistlán de Mejía, Balleza 5604 (HUAZ); Tepechitlán, Balleza 3480 (HUAZ); Villanueva, Balleza 3343 (HUAZ); Zacatecas, Balleza s/n (HUAZ).

${ }^{*}$ Cosmos concolor Sherff

HP. Valparaiso, Rose s/n (Citado en McVaugh, 1984).

Cosmos crithmifolius Kunth

HP. Juchipila, Balleza 8877 (HUAZ); Tabasco, Balleza 9492 (HUAZ); Valparaíso, Balleza 9352 (HUAZ).

${ }^{*}$ Cosmos landii Sherff

HP. Juchipila, Balleza 8874 (HUAZ).

${ }^{*}$ Cosmos linearifolius (Sch. Bip.) Hemsl. var. linearifolius

HP. Valparaíso, Balleza 9341 (HUAZ).

${ }^{*}$ Cosmos montanus Sherff

HP. Valparaiso, Rose s/n (Citado en McVaugh, 1984).

${ }^{*}$ Cosmos palmeri B. L. Rob. var. palmeri

HP. Tlaltenango de Sánchez Roman, Correa 154 (Citado en McVaugh, 1984).

Cosmos parviflorus (Jacq.) Pers.

HA. Chalchihuites, Balleza 7166 (HUAZ); Cuauhtémoc, Del Castillo 37ZA (CHAPA); Jalpa, Balleza 8983 (HUAZ); Jerez, Balleza 6514 (HUAZ); Juchipila, Balleza 9044 (HUAZ); Nochistlán de Mejía, Balleza 9018 (HUAZ); Sain Alto, García 801 (CHAPA); Sombrerete, Balleza 7127 (HUAZ); Tabasco, Balleza 9489 (HUAZ); Teúl de González Ortega, Balleza 7328 (HUAZ); Valparaíso, Balleza 9292 (HUAZ); Zacatecas, Balleza 6973 (HUAZ).

${ }^{*}$ Cosmos scabiosoides Kunth var. scabiosoides

HP. Jalpa, Balleza 6488 (HUAZ); Nochistlán de Mejía, Balleza 8676 (HUAZ).

${ }^{*}$ Cosmos sessilis Sherff

HP. Valparaíso, Balleza 9384 (HUAZ).

Cosmos sulphureus Cav.

HA. García de la Cadena; Balleza 7306 (HUAZ); Juchipila, Balleza 8814 (HUAZ); Moyahua de Estrada, Enríquez 1593 (HUAZ).

${ }^{*}$ Critoniopsis barbinervis (Sch. Bip.) H. Rob.

AR. Monte Escobedo, Balleza 7454 (HUAZ); Tabasco, Balleza 9495 (HUAZ); Valparaíso, Balleza 9403 (HUAZ); Villanueva, Gentry 18268 (HUAZ).

${ }^{*}$ Critoniopsis foliosa (Benth.) H. Rob. [=Vernonia steetzii Sch. Bip.]

AR. Juchipila, Balleza 7824 (HUAZ).

${ }^{*}$ Critoniopsis tomentosa (La Llave) H. Rob. [=Vernonia paniculata DC.]

AR. Jalpa, Balleza 8006a (HUAZ); Juchipila, Balleza 8034 (HUAZ). 
Anexo. Continuación.

${ }^{*}$ Critoniopsis uniflora (Sch. Bip.) H. Rob. [=Vernonia uniflora Sch. Bip.]

AR. Monte Escobedo, García 859 (MEXU); Teúl de González Ortega, Balleza 8062 (HUAZ); Valparaíso, Balleza 7533 (HUAZ); Villanueva, Balleza 3380 (HUAZ).

${ }^{*}$ Critoniopsis villaregalis (Carvajal) H. Rob. [=Vernonia tequilana S. B. Jones \& Stutts]

AR. Juchipila, Enríquez 852 (HUAZ); Moyahua de Estrada, Enríquez 1446 (HUAZ).

Dahlia coccinea Cav.

HP. Jalpa, Balleza 6459 (HUAZ); Jerez, Balleza 6539 (HUAZ); Juchipila, Balleza 8711 (HUAZ); Moyahua de Estrada, Enríquez 1085 (HUAZ); Teúl de González Ortega, Balleza 6380 (HUAZ); Tlaltenango de Sánchez Román, Balleza 6755 (HUAZ); Valparaíso, Balleza 9404 (HUAZ); Zacatecas, Balleza 6987 (HUAZ).

Dahlia pinnata Cav.

HP. Moyahua de Estrada, Enríquez 139 (MEXU); Tlaltenango de Sánchez Román, Balleza 6730 (HUAZ).

Decachaeta incompta (DC.) R. M. King \& H. Rob. [=Eupatorium incomptum DC.]

HP. El Salvador, Balleza 9678b (HUAZ); Juchipila, Balleza 11521 (HUAZ); Moyahua de Estrada, Enríquez 1326 (HUAZ).

Delilia biflora (L.) Kuntze

HA. Juchipila, Balleza 8899 (HUAZ); Moyahua de Estrada, Enríquez 1139 (HUAZ); Tabasco, Balleza 9470 (HUAZ).

Dicranocarpus parviflorus A. Gray

HA. Concepción del Oro, Balleza 6245 (HUAZ); Mazapil, Balleza 9740 (HUAZ); Miguel Auza, Balleza 6899 (HUAZ); Villa de Cos, Balleza 10691 (HUAZ).

${ }^{*}$ Digitacalia jatrophoides (Kunth) Pippen var. jatrophoides

HP. Juchipila, Balleza 9035 (HUAZ); Nochistlán de Mejía, Balleza 7620 (HUAZ); Teúl de González Ortega, Balleza 7313 (HUAZ); Tlaltenango de Sánchez Román, Balleza 6711 (HUAZ).

Dyssodia papposa (Vent.) Hitchc.

HA. Benito Juárez, Balleza 7369 (HUAZ); Chalchihuites, Balleza 7155 (HUAZ); Concepción del Oro, Balleza 6634 (HUAZ); El Salvador, Balleza 9687 (HUAZ); Fresnillo, Balleza 6012 (HUAZ); General Pánfilo Natera, Balleza 7735 (HUAZ); Jerez, Balleza 7073 (HUAZ); Juan Aldama, Balleza 11155 (HUAZ); Juchipila, Balleza 9926 (HUAZ); Mazapil, Balleza 9768 (HUAZ); Nochistlán de Mejía, Balleza 7632 (HUAZ); Noria de Ángeles, Balleza 7763 (HUAZ); Pánuco, Balleza 7648 (HUAZ); Pinos, Balleza 5360 (HUAZ); Río Grande, Balleza 10705 (HUAZ); Sombrerete, Balleza 5775 (HUAZ); Tabasco, Balleza 9457 (HUAZ); Valparaíso, Balleza 6085 (HUAZ); Villa de Cos, Balleza 7691 (HUAZ); Villa González Ortega, Balleza 7751 (HUAZ); Villanueva, Balleza 7256 (HUAZ); Zacatecas, Balleza 7003 (HUAZ).

${ }^{*}$ Dyssodia pinnata (Cav.) B. L. Rob. var. glabrescens Strother

HP. Concepción del Oro, Jansen 469 (MEXU); Sain Alto, Gentry 8544 (MEXU).

*Dyssodia pinnata (Cav.) B. L. Rob. var. pinnata

HP. Fresnillo, Balleza 7228 (HUAZ); Genaro Codina, Balleza 10346 (HUAZ); Jerez, Balleza 6531 
Anexo. Continuación.

(HUAZ); Mazapil, Balleza 10674 (HUAZ); Miguel Auza, Balleza 6910 (HUAZ); Monte Escobedo, Balleza 7416 (HUAZ); Sombrerete, Balleza 7196 (HUAZ); Tepechitlán, Balleza 3496 (HUAZ); Valparaíso, Balleza 7513 (HUAZ); Villa de Cos, Balleza 6815 (HUAZ); Zacatecas, Balleza 6978 (HUAZ).

${ }^{*}$ Dyssodia tagetiflora Lag.

HP. Apozol, Balleza 7278 (HUAZ); Benito Juárez, Balleza 7357 (HUAZ); Fresnillo, Balleza 5653 (HUAZ); García de la Cadena, Balleza 7304 (HUAZ); General Joaquín Amaro, Balleza 5932 (HUAZ); Jalpa, Balleza 7933 (HUAZ); Juchipila, Balleza 9626 (HUAZ); Monte Escobedo, Balleza 5551 (HUAZ); Moyahua de Estrada, Enríquez 1452 (HUAZ); Nochistlán de Mejía, Balleza 5629 (HUAZ); Tabasco, Balleza 5961 (HUAZ); Valparaíso, Balleza 8453 (HUAZ).

Eclipta prostrata (L.) L.

HA. Apozol, Balleza 7277 (HUAZ); Moyahua de Estrada, Balleza 7267b (HUAZ).

${ }^{*}$ Erigeron calcicola Greenm.

HA. Concepción del Oro, Balleza 5502 (HUAZ); Loreto, Balleza 5281 (HUAZ); Mazapil, Balleza 6654 (HUAZ); Pánuco, Balleza 7658 (HUAZ); Pinos, Balleza 5318 (HUAZ); Villa de Cos, Balleza 6816 (HUAZ); Villa González Ortega, Balleza 10566 (HUAZ).

Erigeron delphinifolius Willd.

HA. Chalchihuites, Balleza 7157 (HUAZ); General Joaquín Amaro, Rose 2718 (MEXU); Guadalupe, Balleza 7011 (HUAZ); Jalpa, Balleza 6478 (HUAZ); Jerez, Balleza 6529 (HUAZ); Juchipila, Balleza 7926 (HUAZ); Nochistlán de Mejía, Balleza 7583 (HUAZ); Sombrerete, Balleza 7115 (HUAZ); Teúl de González Ortega, Balleza 6381 (HUAZ); Tlaltenango de Sánchez Román, Balleza 6765 (HUAZ); Valparaíso, Balleza 7506 (HUAZ).

${ }^{*}$ Erigeron exilis A. Gray

HP. Teúl de González Ortega, Balleza 8068b (HUAZ).

Erigeron flagellaris A. Gray

HP. Sombrerete, Nesom 4425 (CHAPA).

${ }^{\star}$ Erigeron janivultus G. L. Nesom

HP. Chalchihuites, Balleza 7170 (HUAZ); Concepción del Oro, Balleza 6222 (HUAZ); Jerez, Balleza 6523 (HUAZ); Juan Aldama, Balleza 5823 (HUAZ); Miguel Auza, Balleza 6876 (HUAZ); Monte Escobedo, Balleza 7466 (HUAZ); Pinos, Balleza 5319 (HUAZ); Sombrerete, Balleza 6155 (HUAZ); Villa de Cos, Balleza 7676 (HUAZ); Villanueva, Balleza 7250 (HUAZ).

Erigeron longipes DC.

HP. Genaro Codina, Balleza 10359 (HUAZ); Juchipila, Balleza 5344 (HUAZ); Miguel Auza, Balleza 6895 (HUAZ); Teúl de González Ortega, Balleza 6383 (HUAZ); Tlaltenango de Sánchez Román, Balleza 8408 (HUAZ).

Erigeron modestus A. Gray

HA. Concepción del Oro, Johnston 11558 (MEXU).

Erigeron pubescens Kunth

HP. Villa de Cos, Balleza 6846 (HUAZ). 
Anexo. Continuación.

${ }^{*}$ Erigeron subacaulis (McVaugh) G. L. Nesom

HP. Jerez, Balleza 5392 (HUAZ); Juchipila, Balleza 10088 (HUAZ); Loreto, Balleza 5289 (HUAZ); Sombrerete, Balleza 8187 (HUAZ); Teúl de González Ortega, Balleza 3396 (HUAZ); Villa González Ortega, Balleza 7753 (HUAZ).

${ }^{*}$ Erigeron velutipes Hook. \& Arn.

HA. Jalpa, McVaugh 25620 (ENCB); Teúl de González Ortega, Balleza 8067 (HUAZ); Tlaltenango de Sánchez Román; Balleza 5263 (HUAZ).

${ }^{\star}$ Erigeron zacatensis G. L. Nesom

HA. Benito Juárez, Balleza 6347 (HUAZ); Fresnillo, Sundberg 2914 (MEXU); Jerez, Balleza 6552 (HUAZ).

*Euphrosyne partheniifolia DC.

HP. Concepción del Oro, Balleza 6264 (HUAZ); Jiménez del Teúl, Balleza 6148 (HUAZ); Mazapil, Balleza 6675 (HUAZ); Río Grande, Balleza 10724 (HUAZ); Valparaíso, Balleza 6067 (HUAZ); Villa de Cos, Balleza 6787 (HUAZ).

${ }^{*}$ Eutetras palmeri A. Gray

HA. Loreto, Balleza 6594 (HUAZ); Zacatecas, Balleza 219 (HUAZ).

${ }^{*}$ Flaveria anomala B. L. Rob.

HA. Concepción del Oro, Balleza 6244 (HUAZ); El Salvador, Balleza 9692 (HUAZ); Mazapil, Balleza 6663 (HUAZ); Miguel Auza, Balleza 6878 (HUAZ).

*Flaveria oppositifolia (DC.) Rydb.

HP. Miguel Auza, Balleza 6890 (HUAZ).

Flaveria trinervia (Spreng.) C. Mohr

HA. Juchipila, Balleza 10868 (HUAZ); Mazapil, Balleza 11292 (HUAZ); Moyahua de Estrada, Enríquez 1287 (HUAZ); Valparaíso, Balleza 6078 (HUAZ); Villa de Cos, Balleza 5429 (HUAZ).

Fleischmannia pycnocephala (Less.) R. M. King \& H. Rob. [=Eupatorium pycnocephalum Less.] HP. Jalpa, Balleza 7563 (HUAZ); Juchipila, Balleza 7849 (HUAZ); Mazapil, Balleza 11060 (HUAZ); Moyahua de Estrada, Enríquez 1389 (HUAZ); Tlaltenango de Sánchez Román, Balleza 8398 (HUAZ); Villa de Cos, Balleza 10655 (HUAZ).

Florestina pedata (Cav.) Cass.

HA. Villa de Cos, Balleza 10615 (HUAZ).

Flourensia cernua DC.

AR. Concepción del Oro, García 8007 (MEXU); El Salvador, Balleza 8300 (HUAZ); General Pánfilo Natera, Balleza 7727 (HUAZ); Mazapil, Balleza 8324 (HUAZ); Pinos, Balleza 10229 (HUAZ); Río Grande, Balleza 10725 (HUAZ); Villa de Cos, Balleza 7669 (HUAZ); Villa González Ortega, Balleza 7746 (HUAZ); Zacatecas, Balleza 703 (HUAZ).

${ }^{*}$ Flourensia dentata S. F. Blake

AR. Miguel Auza, Balleza 6911 (HUAZ); Pánuco, Balleza 7643 (HUAZ); Río Grande, Wendt 2185 (MEXU); Villa de Cos, Dillon 652 (MEXU); Villa González Ortega, Balleza 6606 (HUAZ). 
Anexo. Continuación.

*Gaillardia comosa A. Gray

HA. Concepción del Oro, Balleza 2768 (HUAZ); Fresnillo, Balleza 6010 (HUAZ); General Pánfilo Natera, Gonzáles s/n (CHAPA); Mazapil, Balleza 6235 (HUAZ); Pánuco, Balleza 7660 (HUAZ); Pinos, Balleza 10208 (HUAZ); Sombrerete, Balleza 6154 (HUAZ); Villa de Cos, Balleza 4375 (HUAZ).

Galeana pratensis (Kunth) Rydb.

HA. García de la Cadena, Balleza 7288 (HUAZ); Jalpa, Balleza 6694 (HUAZ); Juchipila, Balleza 8850 (HUAZ).

Galinsoga parviflora Cav.

HA. Genaro Codina, Balleza 10356 (HUAZ); General Joaquín Amaro, Balleza 9526 (HUAZ); Jalpa, Balleza 7565 (HUAZ); Jerez, Balleza 7062 (HUAZ); Juan Aldama, Balleza 11154 (HUAZ); Juchipila, Balleza 7890 (HUAZ); Mazapil, Balleza 9790 (HUAZ); Monte Escobedo, Balleza 3881 (HUAZ); Moyahua de Estrada, Balleza 7272 (HUAZ); Nochistlán de Mejía, Balleza 7627 (HUAZ); Pinos, Balleza 8967 (HUAZ); Río Grande, Balleza 10768 (HUAZ); Sombrerete, Balleza 7197 (HUAZ); Valparaíso, Balleza 9326 (HUAZ); Zacatecas, Balleza 599 (HUAZ).

Galinsoga quadriradiata Ruiz \& Pav.

HA. García de la Cadena, Balleza 7286 (HUAZ); Juchipila, Balleza 9947 (HUAZ); Monte Escobedo, Balleza 7488 (HUAZ); Moyahua de Estrada, Enríquez 1402 (HUAZ); Valparaíso, Balleza 6071 (HUAZ); Villanueva, Balleza 7254 (HUAZ); Zacatecas, Balleza 6993 (HUAZ).

Gamochaeta americana (Mill.) Cabrera [=Gnaphalium americanum Mill.]

HA. General Joaquín Amaro, Rose 3644 (Citado en McVaugh, 1984).

Gamochaeta sphacelata (Kunth) Cabrera [=Gnaphalium sphacelatum Kunth in H.B.K.]

HA. Benito Juárez, Balleza 6326 (HUAZ); Jerez, Balleza 7064 (HUAZ).

Gochnatia hypoleuca (DC.) A. Gray subsp. hypoleuca

HA. Concepción del Oro, Marroquín s/n (ENCB).

*Grindelia inuloides Willd. var. inuloides

HP. Momax, Santana 1253 (MEXU).

${ }^{*}$ Grindelia oxylepis Greene

HP. Chalchihuites, Balleza 6119 (HUAZ); Fresnillo, Balleza 6018 (HUAZ); Genaro Codina, Balleza 10344 (HUAZ); Guadalupe, Balleza 10339 (HUAZ); Jerez, Balleza 6546 (HUAZ); Juchipila, Balleza 7867 (HUAZ); Mazapil, Balleza 6641 (HUAZ); Pinos, Balleza 11326 (HUAZ); Sombrerete, Balleza 6158 (HUAZ); Teúl de González Ortega, Balleza 6377 (HUAZ); Villa de Cos, Balleza 6790 (HUAZ); Villanueva, Villarreal 2335 (CHAPA); Zacatecas, Balleza 4350 (HUAZ).

*Grindelia subdecurrens DC.

HP. Pinos, García s/n (CHAPA).

*Guardiola mexicana Humb. \& Bonpl. var. angustifolia (A. Gray) McVaugh HP. Moyahua de Estrada, Hernández 9620 (MEXU).

*Guardiola mexicana Humb. \& Bonpl. var. mexicana HP. Juchipila, Balleza 8035 (HUAZ). 
Anexo. Continuación.

*Gutierrezia conoidea (Hemsl.) M. A. Lane [=Xanthocephalum conoideum Hemsl.]

HA. Benito Juárez, Balleza 6346 (HUAZ); Chalchihuites, Balleza 7185 (HUAZ); Fresnillo, Balleza 11186 (HUAZ); Genaro Codina, Balleza 10348 (HUAZ); Jerez, Balleza 6509 (HUAZ); Jiménez del Teúl, Balleza 6134 (HUAZ); Monte Escobedo, Balleza 7465 (HUAZ); Nochistlán de Mejía, Balleza 9022 (HUAZ); Río Grande, Balleza 11147 (HUAZ); Sombrerete, Balleza 7088 (HUAZ); Teúl de González Ortega, Balleza 7347 (HUAZ); Valparaíso, Balleza 7507 (HUAZ); Villa de Cos, Balleza 10633 (HUAZ); Villanueva, Balleza 7239 (HUAZ).

Gutierrezia microcephala (DC.) A. Gray

HP. Juan Aldama, Balleza 11153 (HUAZ); Mazapil, Balleza 4057 (HUAZ); Miguel Auza, Balleza 6913 (HUAZ); Sombrerete, Balleza 7144 (HUAZ); Villa de Cos, Banda s/n (CHAPA).

Gutierrezia sarothrae (Pursh.) Britton \& Rusby

AR. Concepción del Oro, sin ejemplar de referencia (citado en Aldape et al., 1996).

*Gutierrezia sericocarpa (A. Gray) M. A. Lane [=Xanthocephalum sericocarpum A. Gray] HP. Benito Juárez, Balleza 6311 (HUAZ); Chalchihuites, Balleza 6124 (HUAZ); Concepción del Oro, Balleza 6247 (HUAZ); Fresnillo, Balleza 5661 (HUAZ); General Francisco Murguía, Balleza 6938 (HUAZ); General Joaquín Amaro, Balleza 5930 (HUAZ); Jerez, Balleza 5382 (HUAZ); Juan Aldama, Balleza 11314 (HUAZ); Mazapil, Balleza 6656 (HUAZ); Monte Escobedo, Balleza 5548 (HUAZ); Río Grande, Balleza 5691 (HUAZ); Sombrerete, Balleza 5770 (HUAZ); Villa de Cos, Balleza 6809 (HUAZ); Zacatecas, Balleza 6974 (HUAZ).

Gutierrezia sphaerocephala A. Gray [=Xanthocephalum sphaerocephalum (A. Gray) Shinners] HP. Mazapil, Chiang s/n (MEXU).

Gymnosperma glutinosum (Spreng.) Less.

AR. Chalchihuites, Balleza 6117 (HUAZ); Concepción del Oro, Balleza 5492 (HUAZ); Cuauhtémoc, Del Castillo 135 ZA (CHAPA); El Salvador, Balleza 9731 (HUAZ); Fresnillo, Balleza 5998 (HUAZ); General Francisco Murguía, Balleza 5888 (HUAZ); General Pánfilo Natera, Balleza 4355 (HUAZ); Juan Aldama, Balleza 5833 (HUAZ); Loreto, Balleza 5286 (HUAZ); Mazapil, Balleza 8337 (HUAZ); Miguel Auza, Balleza 5847 (HUAZ); Noria de Ángeles, Balleza 5295 (HUAZ); Pinos, Balleza 6445 (HUAZ); Río Grande, Balleza 5692 (HUAZ); Sombrerete, Balleza 5793 (HUAZ); Villa de Cos, Balleza 6826 (HUAZ); Villa González Ortega, Balleza 7741 (HUAZ); Villanueva, Balleza 6506 (HUAZ); Zacatecas, Balleza 7002 (HUAZ).

Helenium mexicanum Kunth

HA. Chalchihuites, Balleza 6118 (HUAZ); Cuauhtémoc, Balleza 10390 (HUAZ); El Salvador, Balleza 9700 (HUAZ); Fresnillo, Balleza 5991 (HUAZ); General Francisco Murguía, Balleza 5892 (HUAZ); Guadalupe, Andreasen 701 (MEXU); Loreto, Balleza 6584 (HUAZ); Mazapil, Balleza 6642 (HUAZ); Pinos, Balleza 6405 (HUAZ); Río Grande, Balleza 5702 (HUAZ); Villa de Cos, Balleza 6847 (HUAZ); Villa González Ortega, Balleza 10582 (HUAZ); Zacatecas, Balleza 4347 (HUAZ).

Helenium microcephalum DC. var. ooclinium (A. Gray) Bierner

HA. Mazapil, Chiang 7886 (MEXU).

${ }^{*}$ Helianthella ciliata S. F. Blake HP. Fresnillo, Balleza 6019 (HUAZ). 
Acta Botanica Mexicana (2002), 59: 5-69

Anexo. Continuación.

*Helianthella mexicana A. Gray

HP. Monte Escobedo, Balleza 5557 (HUAZ).

Helianthus annuus L.

HA. Benito Juárez, Balleza 6363 (HUAZ); Concepción del Oro, Balleza 6274 (HUAZ); Fresnillo, Balleza 5815 (HUAZ); Mazapil, Balleza 11119 (HUAZ); Pinos, Balleza 6413 (HUAZ); Villa de Cos, Balleza 10690 (HUAZ).

Helianthus ciliaris DC.

HP. Villa de Cos, Banda 38 (CHAPA).

Helianthus laciniatus A. Gray

HP. Concepción del Oro, Balleza 6265 (HUAZ); El Salvador, Balleza 9728 (HUAZ); Fresnillo, Balleza 5983 (HUAZ); General Francisco Murguía, Balleza 5899 (HUAZ); Juan Aldama, Balleza 5837 (HUAZ); Loreto, Balleza 6589 (HUAZ); Mazapil, Balleza 6674 (HUAZ); Río Grande, Balleza 5703 (HUAZ); Sombrerete, Balleza 5813 (HUAZ); Villa de Cos, Balleza 6807 (HUAZ); Villa González Ortega, Balleza 10584 (HUAZ); Zacatecas, Balleza 596 (HUAZ).

${ }^{*}$ Heliopsis annua Hemsl.

HA. Tlaltenango de Sánchez Román, Balleza 6706 (HUAZ).

Heterosperma pinnatum Cav.

HA. Chalchihuites, Balleza 7165 (HUAZ); Fresnillo, Villa s/n (CHAPA); Genaro Codina, Balleza 10357 (HUAZ); Guadalupe, Balleza 7008 (HUAZ); Jalpa, Balleza 8985 (HUAZ); Jerez, Balleza 7017 (HUAZ); Juan Aldama, Balleza 11157 (HUAZ); Juchipila, Balleza 9069 (HUAZ); Mazapil, Balleza 9761 (HUAZ); Nochistlán de Mejía, Balleza 7633 (HUAZ); Pánuco, Balleza 7642 (HUAZ); Pinos, Balleza 8944 (HUAZ); Río Grande, Balleza 10701 (HUAZ); Sain Alto, Gentry 8543b (MEXU); Sombrerete, Balleza 7108 (HUAZ); Tabasco, Balleza 9471 (HUAZ); Teúl de González Ortega, Balleza 7327 (HUAZ); Valparaíso, Balleza 9387 (HUAZ); Villa de Cos, Balleza 6781 (HUAZ); Villa González Ortega, Balleza 7760 (HUAZ); Villa Hidalgo, Banda 80 (CHAPA); Zacatecas, Balleza 649 (HUAZ).

${ }^{*}$ Heterotheca inuloides Cass. var. rosei B. Wagenkn.

HP. Benito Juárez, Balleza 6342 (HUAZ); Concepción del Oro, Balleza 3988 (HUAZ); Cuauhtémoc, Balleza 10407 (HUAZ); Fresnillo, Balleza 5817 (HUAZ); Genaro Codina, Balleza 5279 (HUAZ); Jerez, Balleza 6561 (HUAZ); Mazapil, Balleza 6293 (HUAZ); Pinos, Balleza 6420 (HUAZ); Sombrerete, Balleza 6185 (HUAZ); Tabasco, Balleza 499 (HUAZ); Teúl de González Ortega, Balleza 6304 (HUAZ); Tlaltenango de Sánchez Román, Balleza 6778 (HUAZ); Valparaíso, Balleza 6105 (HUAZ); Villa de Cos, Balleza 10689 (HUAZ); Zacatecas, Balleza 4348 (HUAZ).

${ }^{*}$ Heterotheca mucronata Harms ex B. L. Turner

HP. Concepción del Oro, Balleza 5496 (HUAZ); General Joaquín Amaro, Balleza 5939 (HUAZ).

Heterotheca subaxillaris (Lam.) Britton \& Rusby var. subaxillaris

HP. General Pánfilo Natera, Gonzáles s/n (CHAPA); Villa Hidalgo, Banda 80a (CHAPA).

Hieracium abscissum Less.

HP. Jalpa, Balleza 6485 (HUAZ); Monte Escobedo, Balleza 7483 (HUAZ); Nochistlán de Mejía, Balleza 7593 (HUAZ); Teúl de González Ortega, Balleza 6382 (HUAZ); Valparaíso, Balleza 9441 (HUAZ); Zacatecas, Balleza 6975 (HUAZ). 
Anexo. Continuación.

Hieracium pringlei A. Gray

HP. Juchipila, Balleza 7923 (HUAZ); Monte Escobedo, Balleza 5553 (HUAZ); Tlaltenango de Sánchez Román, Balleza 8542 (HUAZ); Valparaíso, Balleza 6049 (HUAZ).

Hieracium schultzii Fries

HP. Apozol, Sainz s/n (MEXU).

${ }^{*}$ Hofmeisteria schaffneri (A. Gray) R. M. King \& H. Rob.

HP. Apozol, Sainz s/n (MEXU).

${ }^{*}$ Hybridella globosa (Ortega) Cass. var. myriophylla (Sch. Bip.) Olsen

HA. Calera, Pringle 8914a (MEXU); Fresnillo, Balleza 5978 (HUAZ); Miguel Auza, Balleza 6914 (HUAZ); Pinos, Balleza 8970 (HUAZ); Villa de Cos, Balleza 7675 (HUAZ).

Hymenoxys odorata DC.

HA. Mazapil, Balleza 6657 (HUAZ); Pinos, Balleza 5326 (HUAZ); Villa Hidalgo, Balleza 6448 (HUAZ).

Hymenoxys scaposa (DC.) K. F. Parker var. linearis (Nutt.) K. F. Parker

HP. Fresnillo, Balleza 6009 (HUAZ); Sombrerete, García 870 (CHAPA).

*Iostephane heterophylla (Cav.) Hemsl.

HP. Jalpa, Balleza 6472 (HUAZ); Nochistlán de Mejía, Balleza 8675 (HUAZ); Tlaltenango de Sánchez Román, Balleza 6773 (HUAZ); Valparaíso, Balleza 9434 (HUAZ).

* Iostephane madrensis (S. Watson) Strother

HP. Valparaíso, Balleza 9415 (HUAZ); General Pánfilo Natera, Webster 15493 (MEXU).

*Isocoma hartwegii (A. Gray) Greene

HP. Pinos, Balleza 10218 (HUAZ); Villa Hidalgo, Reveal 3142 (MEXU); Villanueva, Diaz 2391 (CHAPA); Zacatecas, Wagner 4218 (MEXU).

*Isocoma veneta (Kunth) Greene [=Haplopappus venetus (Kunth) S. F. Blake var. hartwegii (A. Gray) McVaugh]

HP. Benito Juárez, Balleza 6343 (HUAZ); Concepción del Oro, Wendt 588 (MEXU); Fresnillo, Balleza 5816 (HUAZ); General Pánfilo Natera, Balleza 7723 (HUAZ); Guadalupe, Balleza 7714 (HUAZ); Mazapil, Balleza 5515 (HUAZ); Pinos, Balleza 6407 (HUAZ); Villa de Cos, Villaseñor 543 (MEXU); Villa González Ortega, Balleza 7740 (HUAZ); Villanueva, Balleza 6503 (HUAZ); Zacatecas, Balleza 6980 (HUAZ).

Iva ambrosiifolia (A. Gray) A. Gray

HP. Valparaíso, Rose 2408 (MEXU).

Iva dealbata A. Gray

HP. El Salvador, Balleza 9699 (HUAZ); General Francisco Murguía, Balleza 6933b (HUAZ); Mazapil, Balleza 6647 (HUAZ); Pinos, Balleza 10153 (HUAZ); Río Grande, Balleza 10745 (HUAZ); Villa de Cos, Balleza 10693 (HUAZ).

*Jaegeria bellidiflora (Moç. \& Sessé ex DC.) A. M. Torres \& Beaman

HA. Benito Juárez, Balleza 6361 (HUAZ). 
Anexo. Continuación.

Jaegeria hirta (Lag.) Less.

HA. Jalpa, Balleza 8973 (HUAZ); Juchipila, Balleza 9553 (HUAZ); Monte Escobedo, Balleza 7472

(HUAZ); Teúl de González Ortega, Balleza 7346 (HUAZ); Valparaíso, Balleza 9330 (HUAZ).

*Jaumea peduncularis (Hook. \& Arn.) Oliver \& Hiern ex Oliver

HP. Moyahua de Estrada, Enríquez 1766 (HUAZ).

Jefea brevifolia (A. Gray) Strother

AR. Concepción del Oro; Balleza 5495 (HUAZ); Mazapil, Balleza 8796 (HUAZ).

Koanophyllon solidaginifolium (A. Gray) R. M. King \& H. Rob. [=Eupatorium solidaginifolium A. Gray] AR. Jalpa, Balleza 7546 (HUAZ); Juchipila, Balleza 7802 (HUAZ); Moyahua de Estrada, Enríquez 802 (HUAZ); Río Grande, Balleza 5719 (HUAZ).

${ }^{*}$ Kyrsteniopsis nelsonii (B. L. Rob.) R. M. King \& H. Rob. [=Eupatorium nelsonii B. L. Rob.] AR. Juchipila, Balleza 8046 (HUAZ).

Lactuca serriola L. [=Lactuca scariola L.]

HP. Nochistlán de Mejía, Balleza 5631 (HUAZ); Pinos, Balleza 6404 (HUAZ); Zacatecas, Balleza 685 (HUAZ).

Laennecia confusa (Cronquist) G. L. Nesom [=Conyza confusa Cronquist]

HP. Benito Juárez, Balleza 7381 (HUAZ); Genaro Codina, Balleza 10375 (HUAZ); Jerez, Balleza 7066 (HUAZ); Monte Escobedo, Balleza 3865 (HUAZ); Pinos, Balleza 10601 (HUAZ); Teúl de González Ortega, Balleza 6357 (HUAZ); Tlaltenango de Sánchez Román, Balleza 6754 (HUAZ); Valparaíso, Balleza 9337 (HUAZ).

Laennecia coulteri (A. Gray) G. L. Nesom

HA. Concepción del Oro, Gonzáles 186 (CHAPA); Mazapil, Balleza 10666 (HUAZ); Río Grande, Balleza 10764 (HUAZ); Sombrerete, Balleza 7146 (HUAZ); Villa de Cos, Balleza 6802 (HUAZ).

Laennecia filaginoides DC. [=Conyza filaginoides (DC.) Hieron.]

HA. Benito Juárez, Balleza 7370 (HUAZ); Jerez, Balleza 6524 (HUAZ); Monte Escobedo, Balleza 7468a (HUAZ); Sombrerete, Balleza 7104 (HUAZ); Tlaltenango de Sánchez Román, Balleza 5262 (HUAZ); Valparaíso, Balleza 7520 (HUAZ); Villanueva, Balleza 7248 (HUAZ).

Laennecia gnaphalioides (Kunth) Cass. [=Conyza gnaphalioides Kunth in H.B.K.] HA. Benito Juárez, Balleza 7371 (HUAZ); Chalchihuites, Balleza 7174 (HUAZ).

*Laennecia microglossa (S. F. Blake) G. L. Nesom [=Conyza dentonae McVaugh] HA. Fresnillo, Balleza 5657 (HUAZ); Guadalupe, Villaseñor 545 (MEXU); Pinos, Balleza 10145 (HUAZ); Sombrerete, Balleza 7205 (HUAZ).

Laennecia schiedeana (Less.) G. L. Nesom [=Conyza schiedeana (Less.) Cronquist] HA. Fresnillo, Balleza 5987 (HUAZ); General Francisco Murguía, Balleza 5878 (HUAZ); Mazapil, Balleza 6650 (HUAZ); Río Grande, Balleza 5690 (HUAZ); Tlaltenango de Sánchez Román, Balleza 6732 (HUAZ); Villa de Cos, Balleza 6819 (HUAZ). 
Anexo. Continuación.

Laennecia sophiifolia (Kunth) G. L. Nesom [=Conyza sophiifolia Kunth]

HA. Apulco, Balleza 5645 (HUAZ); Benito Juárez, Balleza 6328 (HUAZ); Chalchihuites, Balleza 7172 (HUAZ); Fresnillo, Balleza 5977 (HUAZ); General Francisco Murguía, Balleza 6959 (HUAZ); General Joaquín Amaro, Balleza 5922 (HUAZ); Jalpa, Balleza 7555 (HUAZ); Jerez, Balleza 6513 (HUAZ); Juchipila, Balleza 8740 (HUAZ); Miguel Auza, Balleza 6884 (HUAZ); Monte Escobedo, Balleza 5537 (HUAZ); Moyahua de Estrada, Enríquez 79 (HUAZ); Nochistlán de Mejía, Balleza 5626 (HUAZ); Pánuco, Balleza 7645 (HUAZ); Pinos, Balleza 6409 (HUAZ); Sombrerete, Balleza 7207 (HUAZ); Tepechitlán, Balleza 6297b (HUAZ); Teúl de González Ortega, Balleza 6353 (HUAZ); Tlaltenango de Sánchez Román, Balleza 6708 (HUAZ); Villa de Cos, Balleza 6783 (HUAZ); Villa González Ortega, Balleza 10580 (HUAZ); Villanueva, Balleza 7238 (HUAZ); Zacatecas, Balleza 6971 (HUAZ).

*Lagascea angustifolia DC.

AR. Valparaíso, Rzedowski 17633 (MEXU).

Lagascea helianthifolia Kunth var. helianthifolia

AR. Juchipila, Balleza 7879 (HUAZ).

*Lagascea helianthifolia Kunth var. levior (B. L. Rob.) B. L. Rob.

AR. Juchipila, Balleza 7953 (HUAZ); Monte Escobedo, Balleza 7505 (HUAZ).

*Lasianthaea aurea (D. Don) K. M. Becker

HP. Monte Escobedo, Narvaes 37 (CHAPA); Teúl de González Ortega, Balleza 6358 (HUAZ).

*Lasianthaea ceanothifolia (Willd.) K. M. Becker var. ceanothifolia

AR. Apozol, Balleza 7281 (HUAZ); Jalpa, Balleza 7540 (HUAZ); Juchipila, Balleza 8808 (HUAZ); Moyahua de Estrada, Enríquez 1072 (HUAZ); Tabasco, Balleza 9490 (HUAZ); Tepechitlán, Balleza 6296b (HUAZ); Tlaltenango de Sánchez Román, Balleza 6707 (HUAZ); Villanueva, Balleza 3376 (HUAZ).

*Lasianthaea ceanothifolia (Willd.) K. M. Becker var. verbenifolia (DC.) K. M. Becker

AR. Teúl de González Ortega, Balleza 6387 (HUAZ).

*Lasianthaea palmeri (Greenm.) K. M. Becker

HP. Juchipila, Balleza 8721 (HUAZ); Nochistlán de Mejía, Balleza 9033 (HUAZ), Tlaltenango de Sánchez Román, Balleza 6770 (HUAZ).

Leibnitzia seemannii (Sch. Bip.) G. L. Nesom [=Chaptalia seemannii (Sch. Bip.) Hemsl.] HP. Valparaíso, McVaugh 25737 (MEXU).

Machaeranthera gymnocephala (DC.) Shinners [=Aster gymnocephalus (DC.) A. Gray] HP. Fresnillo, Balleza 6008 (HUAZ); General Joaquín Amaro, Balleza 9513 (HUAZ); General Pánfilo Natera, Banda s/n (CHAPA); Jerez, Balleza 6528 (HUAZ); Juan Aldama, Balleza 11160 (HUAZ); Miguel Auza, Balleza 5848 (HUAZ); Nochistlán de Mejía, Balleza 6492 (HUAZ); Sombrerete, Balleza 7103 (HUAZ); Valparaíso, Rose 2497 (MEXU); Villa de Cos, Balleza 7702 (HUAZ); Villanueva, Balleza 7236 (HUAZ); Zacatecas, Balleza 6977 (HUAZ).

Machaeranthera pinnatifida (Hook.) Shinners var. chihuahuana B. L. Turner \& R. L. Hartman HP. Concepción del Oro, Saunders 1945 (MEXU); Pánuco, Villaseñor 206 (MEXU). 
Acta Botanica Mexicana (2002), 59: 5-69

Anexo. Continuación.

Machaeranthera pinnatifida (Hook.) Shinners var. pinnatifida [=Haplopappus spinulosus (Pursh) DC.] HP. Concepción del Oro, Gonzáles 8023 (MEXU); El Salvador, Balleza 8290 (HUAZ); Fresnillo, Balleza 5728 (HUAZ); Genaro Codina, Balleza 5278 (HUAZ); General Francisco Murguía, Balleza 5904 (HUAZ); Jiménez del Teúl, Balleza 6141 (HUAZ); Juan Aldama, Balleza 5839 (HUAZ); Loreto, Balleza 5287 (HUAZ); Mazapil, Balleza 5528 (HUAZ); Miguel Auza, Balleza 5858 (HUAZ); Pinos, Balleza 6442 (HUAZ); Río Grande, Balleza 5707 (HUAZ); Villa de Cos, Balleza 6793 (HUAZ); Villa González Ortega, Balleza 7754 (HUAZ); Villanueva, Balleza 6505 (HUAZ); Zacatecas, Balleza 198 (HUAZ).

Machaeranthera pinnatifida (Hook.) Shinners var. scabrella (Greene) B. L. Turner \& R. L. Hartm. HP. Cuauhtémoc, Del Castillo 74ZA (CHAPA); Fresnillo, Villarreal 2247 (CHAPA); Villa de Cos, Banda 40 (CHAPA).

Machaeranthera tanacetifolia (Kunth) Nees [=Aster tanacetifolius Kunth]

HA. Calera, Banda 1113 (CHAPA); Fresnillo, Balleza 5666 (HUAZ); General Francisco Murguía, Balleza 6925 (HUAZ); General Pánfilo Natera, Balleza 7734 (HUAZ); Guadalupe, Villaseñor 546 (MEXU); Juan Aldama, Balleza 5825 (HUAZ); Mazapil, Chiang 7930 (MEXU); Miguel Auza, Balleza 6902a (HUAZ); Pánuco, Balleza 7656 (HUAZ); Río Grande, Balleza 5711 (HUAZ); Sombrerete, Balleza 5797 (HUAZ); Villa de Cos, Balleza 6796 (HUAZ); Zacatecas, Balleza 4349 (HUAZ).

${ }^{*}$ Matricaria recutita (L.) Rauschert [=Matricaria courrantiana DC.]

HA. General Joaquín Amaro, Rose 2805 (HUAZ).

Melampodium divaricatum (Rich.) DC.

HA. Juchipila, Balleza 7780 (HUAZ); Moyahua de Estrada, Enríquez 1040 (HUAZ).

Melampodium perfoliatum (Cav.) Kunth

HA. Apozol, Enríquez 662 (HUAZ); Benito Juárez, Balleza 6329 (HUAZ); Juchipila, Balleza 9569 (HUAZ); Monte Escobedo, Balleza 7433 (HUAZ); Nochistlán de Mejía, Balleza 5637 (HUAZ); Tepechitlán, Balleza 3481 (HUAZ); Tlaltenango de Sánchez Román, Balleza 4103 (HUAZ); Valparaíso, Balleza 6098 (HUAZ).

Melampodium sericeum Lag.

HA. Jalpa, Balleza 6693 (HUAZ); Juchipila, Balleza 8699 (HUAZ); Nochistlán de Mejía, Balleza 9010 (HUAZ); Río Grande, Balleza 10751 (HUAZ); Tabasco, Balleza 5959 (HUAZ); Valparaíso, Balleza 9391 (HUAZ); Villanueva, Anderson 12625 (MEXU).

Milleria quinqueflora L.

HA. General Joaquín Amaro, Balleza 9533 (HUAZ); Jalpa, Balleza 8996 (HUAZ); Juchipila, Balleza 8810 (HUAZ); Moyahua de Estrada, Enríquez 1630 (HUAZ).

${ }^{*}$ Montanoa leucantha (Lag. \& Segura) S. F. Blake subsp. leucantha

A. Chalchihuites, Balleza 6120 (HUAZ); Fresnillo, Anderson 3586 (MEXU); García de la Cadena, Balleza 7295 (HUAZ); Genaro Codina, Balleza 6567 (HUAZ); General Joaquín Amaro, Rose 3647 (MEXU); Guadalupe, Balleza 7711 (HUAZ); Jalpa, Balleza 6476 (HUAZ); Jerez, Balleza 5420 (HUAZ); Juan Aldama, Bartholomew 3603 (MEXU); Juchipila, Balleza 9063 (HUAZ); Nochistlán de Mejía, Balleza 9002 (HUAZ); Sombrerete, Anderson 3583 (MEXU); Tabasco, Balleza 9506 (HUAZ); Tlaltenango de Sánchez Román, Balleza 6767 (HUAZ); Valparaíso, Balleza 9319 (HUAZ); Villanueva, Cronquist 10273a (MEXU); Zacatecas, Balleza 6984 (HUAZ). 
Anexo. Continuación.

*Montanoa tomentosa Cerv. subsp. tomentosa

AR. Valparaíso, Balleza 9453 (HUAZ).

Nicolletia edwardsii A. Gray

HA. General Francisco Murguía, Balleza 5893 (HUAZ); Mazapil, Balleza 11254 (HUAZ).

*Otopappus tequilanus (A. Gray) B. L. Rob. [=Otopappus tequilanus (A. Gray) B. L. Rob. var. griseus McVaugh]

AR. Juchipila, Balleza 9142 (HUAZ); Moyahua de Estrada, Enríquez 1642 (HUAZ).

Parthenium argentatum A. Gray

AR. Concepción del Oro, Palmer 374 (MEXU); Mazapil, Balleza 6689 (HUAZ); Villa de Cos, Balleza 6828 (HUAZ).

Parthenium bipinnatifidum (Ortega) Rollins

HA. Calera, Rodríguez 1162 (MEXU); Concepción del Oro, Balleza 2960 (HUAZ); Fresnillo, Balleza 5979 (HUAZ); Genaro Codina, Balleza 10355 (HUAZ); General Francisco Murguía, Balleza 6929 (HUAZ); Guadalupe, Balleza 10342 (HUAZ); Juan Aldama, Balleza 5820 (HUAZ); Mazapil, Balleza 8747 (HUAZ); Moyahua de Estrada, Enríquez 1741 (HUAZ); Noria de Ángeles, Balleza 7774 (HUAZ); Pánuco, Balleza 7644 (HUAZ); Pinos, Balleza 6437 (HUAZ); Río Grande, Balleza 5712 (HUAZ); Sombrerete, Tenorio 2172 (MEXU); Valparaíso, Balleza 6023 (HUAZ); Villa de Cos, Balleza 6792 (HUAZ); Villa González Ortega, Balleza 6612 (HUAZ); Zacatecas, Balleza 3921 (HUAZ).

Parthenium confertum A. Gray var. lyratum (A. Gray) Rollins

HA. Concepción del Oro, Gonzáles 8038 (MEXU); Mazapil, Balleza 11048 (HUAZ); Villa de Cos, Balleza 10647 (HUAZ).

Parthenium hysterophorus L.

HA. El Salvador, Balleza 9688 (HUAZ); Juchipila, Balleza 5345 (HUAZ); Moyahua de Estrada, Enríquez 1255 (HUAZ).

Parthenium incanum Kunth

AR. Concepción del Oro, Balleza 5491 (HUAZ); El Salvador, Balleza 9722 (HUAZ); Genaro Codina, Balleza 6565 (HUAZ); General Francisco Murguía, Balleza 6935 (HUAZ); General Pánfilo Natera, Balleza 7729 (HUAZ); Loreto, Balleza 6581 (HUAZ); Mazapil, Balleza 8326 (HUAZ); Miguel Auza, Balleza 5864 (HUAZ); Pánuco, Balleza 7655 (HUAZ); Pinos, Balleza 8962 (HUAZ); Río Grande, Balleza 5697 (HUAZ); Villa de Cos, Balleza 6788 (HUAZ); Villa González Ortega, Balleza 6613 (HUAZ); Zacatecas, Balleza 348 (HUAZ).

${ }^{*}$ Parthenium tomentosum DC. var. stramonium (Greene) Rollins

AR. Valparaíso, Balleza 8463 (HUAZ).

Pectis angustifolia Torr. var. angustifolia

HA. Cañitas de Felipe Pescador, Balleza 11201 (HUAZ); Río Grande, Balleza 10711 (HUAZ).

Pectis angustifolia Torr. var. tenella (DC.) D. J. Keil

HA. Sain Alto, Sundberg 2915 (MEXU). 
Anexo. Continuación.

${ }^{*}$ Pectis propetes Greenm.

HA. Valparaiso, Rose 2436 (Citado en McVaugh, 1984).

Pectis prostrata Cav.

HA. Juchipila, Balleza 10473 (HUAZ); Moyahua de Estrada, Enríquez 1289 (HUAZ); Pinos, García s/n (CHAPA); Tepechitlán, Balleza 6299 (HUAZ); Teúl de González Ortega, Balleza 7405 (HUAZ); Valparaíso, Balleza 9313 (HUAZ); Villa de Cos, Balleza 7689 (HUAZ); Villanueva, Anderson 12623 (MEXU).

${ }^{*}$ Perityle castillonii I. M. Johnst.

HP. Teúl de González Ortega, Balleza 7352 (HUAZ).

${ }^{*}$ Perityle feddemae McVaugh

HP. Juchipila, Balleza 7778 (HUAZ).

${ }^{*}$ Perityle glaucescens B. L. Turner

HP. Juchipila, Panero 6858 (MEXU).

Perityle microglossa Benth. var. microglossa

HA. Moyahua de Estrada, Enríquez 1437 (HUAZ).

${ }^{*}$ Perityle trichodonta S. F. Blake

HP. Valparaíso, McVaugh 25790 (MEXU).

${ }^{*}$ Perymenium buphthalmoides DC. var. tenellum (A. Gray) McVaugh

HP. Chalchihuites, Balleza 6123 (HUAZ); General Joaquín Amaro, Balleza 8652 (HUAZ); Jerez, Balleza 6535 (HUAZ); Monte Escobedo, Balleza 5547 (HUAZ); Tabasco, Balleza 8635 (HUAZ); Tlaltenango de Sánchez Román, Balleza 8537 (HUAZ); Valparaíso, Balleza 9379 (HUAZ).

${ }^{*}$ Perymenium mendezii DC. var. mendezii

AR. Apozol, Hernández 9629 (MEXU); Genaro Codina, Balleza 10370 (HUAZ); General Joaquín Amaro, Balleza 5934 (HUAZ); Jalpa, Balleza 6698 (HUAZ); Jerez, Balleza 6519 (HUAZ); Jiménez del Teúl, Balleza 7193 (HUAZ); Juchipila, Balleza 8579 (HUAZ); Loreto, Balleza 6590 (HUAZ); Moyahua de Estrada, Enríquez 473 (HUAZ); Noria de Ángeles, Balleza 7764 (HUAZ); Pinos, Balleza 10163 (HUAZ); Sombrerete, Balleza 7118 (HUAZ); Tlaltenango de Sánchez Román, Balleza 8532 (HUAZ); Valparaíso, Balleza 6051 (HUAZ); Villa García, Martínez s/n (MEXU); Villa Hidalgo, Balleza 5372 (HUAZ); Villanueva, Breedlove 61562 (MEXU); Zacatecas, Balleza 6985 (HUAZ).

${ }^{*}$ Perymenium subsquarrosum B. L. Rob. \& Greenm.

AR. General Joaquín Amaro, Rose $2751(\mathrm{GH})$; Juchipila, Balleza 9228 (HUAZ); Valparaíso, McVaugh 17700 (ENCB).

${ }^{*}$ Pinaropappus diguetii McVaugh

HP. Benito Juárez, Balleza 6350 (HUAZ); Valparaíso, Balleza 8452 (HUAZ).

${ }^{*}$ Pinaropappus roseus (Less.) Less. var. maculatus McVaugh

HP. Zacatecas, Wagner 4216 (MEXU). 
Anexo. Continuación.

Pinaropappus roseus (Less.) Less. var. roseus

HP. Chalchihuites, Balleza 6128 (HUAZ); Concepción del Oro, Balleza 5506 (HUAZ); Genaro Codina, Balleza 10347 (HUAZ); General Joaquín Amaro, Balleza 5933 (HUAZ); Guadalupe, Balleza 7004 (HUAZ); Jerez, Balleza 5386 (HUAZ); Juan Aldama, Balleza 5831 (HUAZ); Mazapil, Balleza 8785 (HUAZ); Miguel Auza, Balleza 6872 (HUAZ); Monte Escobedo, Balleza 5549 (HUAZ); Nochistlán de Mejía, Balleza 5600 (HUAZ); Pinos, Balleza 5363 (HUAZ); Río Grande, Balleza 11148 (HUAZ); Sombrerete, Balleza 5801 (HUAZ); Tlaltenango de Sánchez Román, Balleza 8526 (HUAZ); Villa Hidalgo, Balleza 10595 (HUAZ); Villanueva, Balleza 6504 (HUAZ); Zacatecas, Balleza 6979 (HUAZ).

${ }^{*}$ Pippenalia delphinifolia (Rydb.) McVaugh

HP. Moyahua de Estrada, Balleza 4328 (HUAZ); Tlaltenango de Sánchez Román, Panero 6174 (MEXU); Valparaíso, Balleza 9420 (HUAZ).

*Piptothrix pubens A. Gray

AR. Monte Escobedo, Balleza 7474 (HUAZ).

*Piqueria laxiflora B. L. Rob. \& Seaton

HP. Monte Escobedo, Barrie 1132 (MEXU); Pinos, Liston 614-2 (MEXU); Benito Juárez, Balleza 6315 (HUAZ); Teúl de González Ortega, Balleza 6389 (HUAZ); Villanueva, Balleza 3448 (HUAZ).

*Piqueria triflora Hemsl.

HA. Benito Juárez, Balleza 6315 (HUAZ); Juchipila, Balleza 7806 (HUAZ); Teúl de González Ortega, Balleza 7338 (HUAZ); Villanueva, Balleza 3448 (HUAZ).

Piqueria trinervia Cav.

HP. Benito Juárez, Balleza 7360 (HUAZ); Chalchihuites, Balleza 6129 (HUAZ); Fresnillo, Balleza 5658 (HUAZ); Genaro Codina, Balleza 10385 (HUAZ); General Joaquín Amaro, Balleza 5938 (HUAZ); Guadalupe, Balleza 7718 (HUAZ); Jalpa, Balleza 8997 (HUAZ); Jerez, Balleza 6547 (HUAZ); Loreto, Balleza 6592 (HUAZ); Monte Escobedo, Balleza 7450 (HUAZ); Nochistlán de Mejía, Balleza 7572 (HUAZ); Pinos, Balleza 6421 (HUAZ); Sombrerete, Balleza 7200 (HUAZ); Teúl de González Ortega, Balleza 6352 (HUAZ); Villa García, Martínez 493 (MEXU); Villa González Ortega, Balleza 7749 (HUAZ); Zacatecas, Balleza 6966 (HUAZ).

*Pittocaulon filare (McVaugh) H. Rob. \& Brettell [=Senecio filaris McVaugh]

AR. Jalpa, Balleza 6449 (HUAZ).

*Pittocaulon praecox (Cav.) H. Rob. \& Brettell [=Senecio praecox (Cav.) DC.]

AR. Genaro Codina, Balleza 10387 (HUAZ); Loreto, Balleza 8120 (HUAZ); Pinos, Balleza 8122 (HUAZ); Valparaíso, McVaugh 12021 (MEXU); Villanueva, Balleza 6508 (HUAZ).

*Pittocaulon velatum (Greenm.) H. Rob. \& Brettell var. velatum [Senecio praecox (Cav.) DC. var. morelensis (Miranda) McVaugh]

AR. Juchipila, Balleza 10564 (HUAZ).

Pluchea salicifolia (Mill.) S. F. Blake

AR. Juchipila, Faudón s/n (CHAPA); Moyahua de Estrada, Enríquez 1743 (HUAZ).

*Porophyllum coloratum (Kunth) DC. var. obtusifolium (DC.) McVaugh

HA. Monte Escobedo, García 848 (MEXU); Valparaíso, Feddema s/n (MEXU). 
Anexo. Continuación.

${ }^{*}$ Porophyllum linaria (Cav.) DC.

HP. García de la Cadena, Balleza 7301 (HUAZ); Juchipila, Balleza 7791 (HUAZ); Moyahua de Estrada, Enríquez 744 (HUAZ).

Porophyllum macrocephalum DC. [=Porophyllum ruderale (Jacq.) Cass. var. macrocephalum (DC.) Cronquist]

HA. Juchipila, Balleza 9128 (HUAZ); Moyahua de Estrada, Enríquez 1299 (HUAZ); Tepechitlán, Balleza 3488, (HUAZ).

${ }^{*}$ Porophyllum pringlei B. L. Rob.

HA. Moyahua de Estrada, Enríquez 1397 (HUAZ).

Porophyllum scoparium A. Gray

HP. Concepción del Oro, Balleza 5494 (HUAZ); Mazapil, Johnston 11531 (MEXU).

${ }^{*}$ Psacalium amplum (Rydb.) H. Rob. \& Brettell [=Odontotrichum amplum Rydb.]

HP. Benito Juárez, Balleza 7372 (HUAZ); Jerez, Balleza 7014 (HUAZ); Juchipila, Balleza 8867 (HUAZ); Monte Escobedo, Balleza 7423 (HUAZ); Tabasco, Balleza 9488 (HUAZ); Teúl de González Ortega, Balleza 7344 (HUAZ); Tlaltenango de Sánchez Román, Panero 6172 (MEXU); Valparaíso, Balleza 7530 (HUAZ); Villanueva, Villaseñor 1323 (MEXU).

${ }^{*}$ Psacalium laxiflorum Benth.

HP. Valparaiso, Rose 2337 (Citado en McVaugh, 1984).

${ }^{*}$ Psacalium megaphyllum (B. L. Rob. \& Greenm.) Rydb.

HP. García de la Cadena, Balleza 7283 (HUAZ); General Joaquín Amaro, Balleza 9534 (HUAZ); Juchipila, Balleza 9667 (HUAZ); Nochistlán de Mejía, Balleza 7630 (HUAZ).

${ }^{*}$ Psacalium pachyphyllum (Sch. Bip.) H. Rob. \& Brettell [=Odontotrichum pachyphyllum (Sch. Bip.) Rydb.]

HP. Monte Escobedo, Acosta 441 (ENCB).

${ }^{*}$ Psacalium palmeri (Greene) H. Rob. \& Brettell [=Odontotrichum palmeri (Greene) Rydb.] HP. Teúl de González Ortega, Balleza 6384 (HUAZ).

${ }^{*}$ Psacalium peltatum (Kunth) Cass. var. peltatum

HP. Jalpa, Rzedowski \& McVaugh 958 (ENCB), Sombrerete, Enríquez 2214 (HUAZ).

${ }^{*}$ Psacalium peltigerum (B. L. Rob. \& Seat.) Rydb. var. peltigerum

HP. Juchipila, Balleza 8613 (HUAZ); Moyahua de Estrada, Enríquez 61 (HUAZ).

${ }^{*}$ Psacalium platylepis (B. L. Rob. \& Seaton) H. Rob. \& Brettell [=Odontotrichum platylepis (B. L. Rob. \& Seaton) Rydb.]

HP. Juchipila, Balleza 7817 (HUAZ); Tabasco, Balleza 9491 (HUAZ).

${ }^{*}$ Psacalium poculiferum (S. Watson) Rydb.

HP. Huanusco, Correa 85 (MICH). 
Anexo. Continuación.

*Psacalium sinuatum (Cerv.) H. Rob. \& Brettell [=Odontotrichum sinuatum (Cerv.) Rydb.]

HP. Benito Juárez, Balleza 6362 (HUAZ); Chalchihuites, Balleza 7177 (HUAZ); General Joaquín Amaro, Balleza 9512 (HUAZ); Jalpa, Balleza 8994 (HUAZ); Jerez, Balleza 7060 (HUAZ); Monte Escobedo, Balleza 7462 (HUAZ); Nochistlán de Mejía, Balleza 7589 (HUAZ); Sombrerete, Balleza 6170 (HUAZ); Teúl de González Ortega, Balleza 7343 (HUAZ); Tlaltenango de Sánchez Román, Balleza 6759 (HUAZ); Valparaíso, Balleza 7515 (HUAZ); Villanueva, Balleza 7253 (HUAZ); Zacatecas, Balleza 6969 (HUAZ).

Pseudognaphalium attenuatum (DC.) Anderb. var. attenuatum [=Gnaphalium attenuatum DC.] HP. Guadalupe, Balleza 7719 (HUAZ); Juchipila, Balleza 7820 (HUAZ); Sombrerete, Balleza 7113 (HUAZ).

Pseudognaphalium bicolor Bioletti

HP. Nochistlán de Mejía, Balleza 7621 (HUAZ).

Pseudognaphalium brachypterum (DC.) Anderb.

HP. Juchipila, Balleza 9549 (HUAZ); Monte Escobedo, Balleza 3902 (HUAZ); Tlaltenango de Sánchez Román, Balleza 6734 (HUAZ).

Pseudognaphalium canescens (DC.) Anderb. [=Gnaphalium canescens DC.]

HA. Benito Juárez, Balleza 7383 (HUAZ); Chalchihuites, Balleza 7173 (HUAZ); García de la Cadena, Balleza 7294 (HUAZ); Jerez, Balleza 7034 (HUAZ); Juchipila, Balleza 7836 (HUAZ); Monte Escobedo, Balleza 7427 (HUAZ); Nochistlán de Mejía, Balleza 7574 (HUAZ); Pinos, Balleza 11323 (HUAZ); Sombrerete, Balleza 7213 (HUAZ); Tlaltenango de Sánchez Román, Rzedowski \& McVaugh 956 (MEXU); Valparaíso, Balleza 7519 (HUAZ); Villa González Ortega, Balleza 7747 (HUAZ).

*Pseudognaphalium chartaceum (Greenm.) Anderb. [=Gnaphalium chartaceum Greenm.] HP. Nochistlán de Mejía, Balleza 7591 (HUAZ); Valparaíso, Balleza 7525 (HUAZ).

*Pseudognaphalium conoideum (Kunth) Anderb.

HP. Fresnillo, Balleza 5649 (HUAZ).

Pseudognaphalium greenmanii (S. F. Blake) Anderb. [=Gnaphalium greenmanii S. F. Blake] HP. Juchipila, Balleza 10096 (HUAZ); Valparaíso, Balleza 9336 (HUAZ).

${ }^{*}$ Pseudognaphalium inornatum (DC.) Anderb. [=Gnaphalium inornatum DC.]

HA. Benito Juárez, Balleza 7375 (HUAZ); Chalchihuites, Balleza 6127 (HUAZ); Fresnillo, Balleza 5992 (HUAZ); General Joaquín Amaro, Balleza 5943 (HUAZ); Jalpa, Balleza 6482 (HUAZ); Jerez, Balleza s/n (HUAZ); Miguel Auza, Balleza 6886 (HUAZ); Pinos, Balleza 10144 (HUAZ); Sombrerete, Balleza 6161 (HUAZ); Tlaltenango de Sánchez Román, Balleza 8405 (HUAZ); Valparaíso, Balleza 7508 (HUAZ); Villa de Cos, Balleza 7672 (HUAZ); Villanueva, Balleza 7235 (HUAZ); Zacatecas, Balleza 669 (HUAZ).

Pseudognaphalium oxyphyllum (DC.) Kirp. var. oxyphyllum

HA. Tlaltenango de Sánchez Román, Rzedowski \& McVaugh 955 (MEXU).

Pseudognaphalium semiamplexicaule (DC.) Anderb.

HP. Guadalupe, García 276 (CHAPA). 
Anexo. Continuación.

${ }^{*}$ Pseudognaphalium semilanatum (DC.) Anderb. [=Gnaphalium semilanatum (DC.) McVaugh]

HA. Tlaltenango de Sánchez Román, Rzedowski \& McVaugh 1036 (MEXU).

Pseudognaphalium stramineum Kunth [=Gnaphalium stramineum Kunth]

HA. Jerez, Balleza 7061 (HUAZ).

Pseudognaphalium viscosum (Kunth) Anderb. [=Gnaphalium viscosum Kunth]

HA. Benito Juárez, Balleza 6312 (HUAZ); Chalchihuites, Balleza 6126 (HUAZ); Jerez, Balleza 6553 (HUAZ); Nochistlán de Mejía, Balleza 6493 (HUAZ); Pinos, Balleza 6417 (HUAZ); Sombrerete, Balleza 6165 (HUAZ); Teúl de González Ortega, Balleza 6307 (HUAZ); Zacatecas, Balleza 611 (HUAZ).

Psilactis asteroides A. Gray [=Aster boltoniae Greene]

HA. Apulco, Balleza 5646 (HUAZ); Chalchihuites, Balleza 6130 (HUAZ); Fresnillo, Balleza 5981 (HUAZ); General Joaquín Amaro, Balleza 5944 (HUAZ); Pinos, Balleza 10178a (HUAZ); Sombrerete, Balleza 6197 (HUAZ); Tlaltenango de Sánchez Román, Balleza 8399 (HUAZ).

Psilactis brevilingulata Sch. Bip. ex Hemsl. [=Aster brevilingulatus (Hemsl.) McVaugh] HA. Cuauhtémoc, Balleza 10413 (HUAZ); Fresnillo, Balleza 5669 (HUAZ); General Joaquín Amaro, Balleza 5921, (HUAZ); Jalpa, Balleza 5246 (HUAZ); Juan Aldama, Balleza 11312 (HUAZ); Mazapil, Balleza 10668 (HUAZ); Nochistlán de Mejía, Balleza 5630 (HUAZ); Pinos, Balleza 6436 (HUAZ); Río Grande, Balleza 5706 (HUAZ); Sombrerete, Balleza 5807 (HUAZ); Tlaltenango de Sánchez Román, Balleza 8401 (HUAZ); Valparaíso, Balleza 6027 (HUAZ); Villa de Cos, Balleza 7677 (HUAZ); Villa González Ortega, Balleza 10578 (HUAZ); Villanueva, Balleza 6499 (HUAZ); Zacatecas, Balleza s/n (HUAZ).

Psilactis tenuis S. Watson

HA. Villa de Cos, García 322 (CHAPA).

Psilostrophe gnaphalodes DC. var. gnaphalodes

HP. Concepción del Oro, Balleza 5499 (HUAZ); El Salvador, Balleza 8282 (HUAZ); Fresnillo, Balleza 5752 (HUAZ); Mazapil, Balleza 6615 (HUAZ); Sombrerete, Tenorio 2166 (MEXU); Villa de Cos, Balleza 6842 (HUAZ).

Pyrrhopappus pauciflorus (D. Don) DC.

HP. Fresnillo, Balleza 5990 (HUAZ); Miguel Auza, Balleza 5870 (HUAZ); Tlaltenango de Sánchez Román, Balleza 8409 (HUAZ).

Ratibida columnifera (Nutt.) Wooton \& Standl. var. columnifera HP. El Salvador, Balleza 9729 (HUAZ); Mazapil, Balleza 10662 (HUAZ).

${ }^{*}$ Roldana chapalensis (S. Watson) H. Rob. \& Brettell [=Senecio chapalensis S. Watson] AR. Juchipila, Balleza 438 (HUAZ); Nochistlán de Mejía, Balleza 7592 (HUAZ); Valparaíso, Balleza 9445 (HUAZ).

Roldana hartwegii (Benth.) H. Rob. \& Brettell [=Senecio hartwegii Benth.] HP. Jalpa, Rzedowski \& McVaugh 939 (ENCB). 
Anexo. Continuación.

${ }^{*}$ Roldana heracleifolia (Hemsl.) H. Rob. \& Brettell [=Senecio heracleifolius Hemsl.]

HP. Tepechitlán, Balleza 3491 (HUAZ); Valparaíso, Balleza 7502 (HUAZ); Villanueva, Balleza 9822 (HUAZ).

*Roldana lineolata (DC.) H. Rob. \& Brettell [=Senecio sinuatus Kunth]

HP. García de la Cadena, Balleza 7282 (HUAZ); Juchipila, Balleza 11640 (HUAZ); Tabasco, Balleza 9474 (HUAZ); Teúl de González Ortega, Balleza 7408 (HUAZ).

${ }^{*}$ Roldana pennellii H. Rob. \& Brettell var. durangensis H. Rob. \& Brettell HP. Monte Escobedo, Balleza 3891 (HUAZ); Nochistlán de Mejía, Balleza 7610 (HUAZ).

${ }^{*}$ Roldana sessilifolia (Hook. \& Arn.) H. Rob. \& Brettell [=Pericalia sessilifolia (Hook. \& Arn.) Rydb.] HP. Benito Juárez, Balleza 7378 (HUAZ); García de la Cadena, Balleza 7285 (HUAZ); Jerez, Balleza 7039 (HUAZ); Juchipila, Balleza 7826 (HUAZ); Monte Escobedo, Balleza 7440 (HUAZ); Nochistlán de Mejía, Balleza 7613 (HUAZ); Tabasco, Balleza 9479 (HUAZ); Teúl de González Ortega, Balleza 7319 (HUAZ); Valparaíso, Balleza 7529 (HUAZ); Villanueva, Balleza 3409 (HUAZ).

*Sanvitalia angustifolia Engelm. ex A. Gray

HA. Apozol, Balleza 7279 (HUAZ); Cuauhtémoc, Del Castillo 53ZA (CHAPA); El Salvador, Balleza 9675b (HUAZ); García de la Cadena, Balleza 7289 (HUAZ); General Francisco Murguía, Balleza 6934 (HUAZ); Jerez, Balleza 7072 (HUAZ); Juchipila, Balleza 10860 (HUAZ); Mazapil, Balleza 8748 (HUAZ); Pinos, Balleza 8945 (HUAZ); Río Grande, Balleza 10720 (HUAZ); Sombrerete, Balleza 7081 (HUAZ); Villa de Cos, Balleza 6782 (HUAZ); Zacatecas, Balleza 595 (HUAZ).

Sanvitalia ocymoides DC.

HA. Fresnillo, Balleza 5755 (HUAZ).

Sanvitalia procumbens Lam.

HA. Genaro Codina, Balleza 10343 (HUAZ); General Enríque Estrada, Gillet 17014 (MEXU); General Francisco Murguía, Balleza 6924 (HUAZ); Guadalupe, Balleza 10338 (HUAZ); Huanusco, Hernández 9646 (MEXU); Juan Aldama, Balleza 5821 (HUAZ); Mazapil, Balleza 9791 (HUAZ); Miguel Auza, Balleza 5856 (HUAZ); Moyahua de Estrada, Balleza 7264b (HUAZ); Noria de Ángeles, Balleza 7765 (HUAZ); Pánuco, Balleza 7649 (HUAZ); Pinos, Balleza 10192 (HUAZ); Río Grande, Balleza 5705 (HUAZ); Sombrerete, Balleza 7202 (HUAZ); Tabasco, Balleza 9472 (HUAZ); Tlaltenango de Sánchez Román, Hernández 9377 (MEXU); Valparaíso, Balleza 6022 (HUAZ); Villa de Cos, Balleza 10316 (HUAZ); Villa González Ortega, Balleza 6608 (HUAZ); Villanueva, Balleza 6507 (HUAZ); Zacatecas, Pringle 1759 (MEXU).

*Sartwellia mexicana A. Gray

HA. Concepción del Oro, Marroquín 3945 (MEXU); Mazapil, Chiang 7925a (MEXU).

Schkuhria pinnata (Lam.) Kuntze var. virgata (La Llave) Heiser

HA. Benito Juárez, Balleza 6333 (HUAZ); Chalchihuites, Balleza 7163 (HUAZ); Fresnillo, Balleza 11192 (HUAZ); García de la Cadena, Balleza 7292 (HUAZ); Genaro Codina, Balleza 10361 (HUAZ); General Joaquín Amaro, Rose 2748 (MEXU); Jalpa, Balleza 6695 (HUAZ); Jerez, Balleza 7018 (HUAZ); Juchipila, Balleza 8738 (HUAZ); Miguel Auza, Balleza 6906 (HUAZ); Monte Escobedo, Balleza 7467 (HUAZ); Nochistlán de Mejía, Balleza 7609 (HUAZ); Pinos, Balleza 6410 (HUAZ); Sombrerete, Balleza 7109 (HUAZ); Tabasco, Balleza 9476 (HUAZ); Tlaltenango de Sánchez Román, Balleza 6762 (HUAZ); Valparaíso, Balleza 7512 (HUAZ); Villanueva, Balleza 7243 (HUAZ); Zacatecas, Balleza 7001 (HUAZ). 
Anexo. Continuación.

${ }^{*}$ Schkuhria schkuhrioides (Link \& Otto) Thell.

HA. Pinos, Balleza 10182 (HUAZ).

Sclerocarpus uniserialis (Hook.) Benth. \& Hook. f. ex Hemsl. var. frutescens (Brandegee) Feddema HA. Moyahua de Estrada, Enríquez 1168 (HUAZ).

Senecio flaccidus Less. var. flaccidus

HP. Fresnillo, Balleza 5650 (HUAZ); Genaro Codina, Balleza 10349 (HUAZ); Guadalupe, Whittemore s/n (MEXU); Jerez, Balleza 8093 (HUAZ); Mazapil, Balleza 6232 (HUAZ); Pinos, Balleza 10244 (HUAZ); Río Grande, Johnston 10425 (MEXU); Villa de Cos, Balleza 6855 (HUAZ); Villa González Ortega, Balleza 10587 (HUAZ); Villanueva, Balleza 9852 (HUAZ); Zacatecas, Balleza 6976 (HUAZ).

*Senecio pseudopicridis T. M. Barkley

HP. Concepción del Oro, Henrickson 13345 (LL-TEX)

*Senecio stoechadiformis DC.

HP. Juchipila, Balleza 7929 (HUAZ); Tabasco, Balleza 9485 (HUAZ); Tlaltenango de Sánchez Román, Balleza 6727 (HUAZ).

Simsia amplexicaulis (Cav.) Pers.

HA. Benito Juárez, Balleza 7396 (HUAZ); Chalchihuites, Balleza 7184 (HUAZ); Concepción del Oro, Balleza 6632 (HUAZ); Cuauhtémoc, Balleza 10410 (HUAZ); El Salvador, Balleza 9691 (HUAZ); Fresnillo, Balleza 5976 (HUAZ); General Joaquín Amaro, Balleza 9529, (HUAZ); Guadalupe, García 805 (CHAPA); Jerez, Balleza 6542 (HUAZ); Juchipila, Balleza 7870 (HUAZ); Mazapil, Balleza 10679 (HUAZ); Miguel Auza, Balleza 6905 (HUAZ); Monte Escobedo, Balleza 7439 (HUAZ); Nochistlán de Mejía, Balleza 7607 (HUAZ); Noria de Ángeles, Balleza 7762 (HUAZ); Pánuco, Balleza 7646 (HUAZ); Pinos, Balleza 6434 (HUAZ); Río Grande, Balleza 10769 (HUAZ); Sombrerete, Balleza 6195 (HUAZ); Tabasco, Balleza 5955 (HUAZ); Valparaíso, Balleza 9282 (HUAZ); Villanueva, Balleza 7242 (HUAZ); Zacatecas, Balleza 25 (HUAZ).

Simsia foetida (Cav.) S. F. Blake var. foetida

HA. Juchipila, Balleza 8851 (HUAZ); Moyahua de Estrada, Enríquez 1707 (HUAZ); Tabasco, Balleza 5956 (HUAZ).

Simsia lagasciformis DC.

HA. Apozol, Balleza 7280 (HUAZ); Jalpa, Balleza 7541 (HUAZ); Juchipila, Balleza 11567 (HUAZ); Moyahua de Estrada, Enríquez 1390 (HUAZ); Villanueva, Balleza 3601 (HUAZ).

*Sinclairia angustissima (A. Gray) B. L. Turner [=Liabum angustissimum A. Gray] HP. Juchipila, Balleza 8595 (HUAZ).

*Sinclairia palmeri (A. Gray) B. L. Turner [=Liabum palmeri A. Gray]

HP. Juchipila, Balleza 9073 (HUAZ); Monte Escobedo, Balleza 7420 (HUAZ); Teúl de González Ortega, Balleza 6386 (HUAZ); Valparaíso, Balleza 9345 (HUAZ).

Smallanthus maculatus (Cav.) H. Rob. var. maculatus [=Polymnia maculata Cav.] HP. General Joaquín Amaro, Rose 2749 (HUAZ). 
Anexo. Continuación.

Solidago scabrida DC.

HP. Concepción del Oro, Balleza 6266 (HUAZ); El Salvador, Balleza 8287 (HUAZ); Fresnillo, Balleza 5671 (HUAZ); General Pánfilo Natera, Balleza 4364 (HUAZ); Mazapil, Balleza 9755 (HUAZ); Miguel Auza, Balleza 6891 (HUAZ); Pinos, Balleza 6438 (HUAZ); Río Grande, Balleza 10766 (HUAZ); Sombrerete, Balleza 6150 (HUAZ); Villa de Cos, Balleza 7665 (HUAZ); Villa González Ortega, Balleza 10585 (HUAZ); Zacatecas, Balleza 702 (HUAZ).

Sonchus oleraceus L.

HA. Benito Juárez, Balleza 6322 (HUAZ); Chalchihuites, Balleza 8195 (HUAZ); Concepción del Oro, Balleza 8023 (HUAZ); El Salvador, Balleza 9683 (HUAZ); Fresnillo, Balleza 5665 (HUAZ); Genaro Codina, Balleza 10362 (HUAZ); General Francisco Murguía, Balleza 6953 (HUAZ); Guadalupe, Balleza 5273 (HUAZ); Jalpa, Balleza 8674 (HUAZ); Jerez, Balleza 5400 (HUAZ); Juchipila, Balleza 9567 (HUAZ); Mazapil, Balleza 11141 (HUAZ); Miguel Auza, Balleza 8110 (HUAZ); Nochistlán de Mejía, Balleza 8010 (HUAZ); Pinos, Balleza 10219 (HUAZ); Río Grande, Balleza 8099 (HUAZ); Sombrerete, Balleza 5803 (HUAZ); Valparaíso, Balleza 6036 (HUAZ); Villa de Cos, Balleza 5421 (HUAZ); Zacatecas, Balleza 10 (HUAZ).

Stevia caracasana DC.

HP. Juchipila, Balleza 10014 (HUAZ); Nochistlán de Mejía, Balleza 7606 (HUAZ); Teúl de González Ortega, Balleza 3394 (HUAZ).

*Stevia dictyophylla B. L. Rob.

AR. Jalpa, Balleza 5242 (HUAZ); Teúl de González Ortega, Balleza 8073 (HUAZ).

*Stevia eupatoria (Spreng.) Willd.

HP. Sombrerete, Breedlove 58949 (MEXU).

*Stevia jaliscensis B. L. Rob.

AR. Valparaiso; Rzedowski 17637 (MEXU).

Stevia jorullensis Kunth

HP. Juchipila, Balleza 9860 (HUAZ).

Stevia lucida Lag. var. lucida

AR. García de la Cadena, Gentry 8524b (MEXU); Genaro Codina, Balleza 6572 (HUAZ); General Joaquín Amaro, Balleza 5340 (HUAZ); Jalpa, Balleza 6480 (HUAZ); Jerez, Balleza 6534 (HUAZ); Juchipila, Balleza 7822 (HUAZ); Monte Escobedo, Balleza 3893 (HUAZ); Nochistlán de Mejía, Balleza 7588 (HUAZ); Sombrerete, Balleza 6166 (HUAZ); Tabasco, Balleza 8275 (HUAZ); Tlaltenango de Sánchez Román, Balleza 8396 (HUAZ); Valparaíso, Balleza 6047 (HUAZ); Zacatecas, Balleza 6991 (HUAZ).

*Stevia micradenia B. L. Rob.

HP. Tlaltenango de Sánchez Román, Rzedowski \& McVaugh 970 (MEXU).

Stevia micrantha Lag.

HA. Chalchihuites, Balleza 7153 (HUAZ); Jalpa, Balleza 7564 (HUAZ); Juan Aldama, Balleza 11180 (HUAZ); Juchipila, Balleza 9055 (HUAZ); Noria de Ángeles, Balleza 7772 (HUAZ); Tabasco, Balleza 9460 (HUAZ); Teúl de González Ortega, Balleza 7407 (HUAZ); Tlaltenango de Sánchez Román, Rzedowski \& McVaugh 970 (MEXU); Villanueva, Cronquist 10270 (MEXU). 
Anexo. Continuación.

*Stevia origanoides Kunth

HP. Benito Juárez, Balleza 7361 (HUAZ); Jerez, Balleza 7023 (HUAZ); Juchipila, Balleza 9884 (HUAZ); Monte Escobedo, Balleza 7444 (HUAZ); Moyahua de Estrada, Enríquez 1391 (HUAZ); Teúl de González Ortega, Balleza 3382 (HUAZ); Valparaíso, Balleza 7509 (HUAZ); Villanueva, Balleza 3454 (HUAZ).

Stevia ovata Willd. var. ovata

HP. Benito Juárez, Balleza 7364 (HUAZ); García de la Cadena, Balleza 7293 (HUAZ); General Joaquín Amaro, Balleza 9514 (HUAZ); Jerez, Balleza 7074 (HUAZ); Juchipila, Balleza 7796 (HUAZ); Monte Escobedo, Balleza 7419 (HUAZ); Moyahua de Estrada, Balleza 7274 (HUAZ); Nochistlán de Mejía, Balleza 7611 (HUAZ); Sombrerete, Balleza 7145 (HUAZ); Tabasco, Balleza 9462 (HUAZ); Tepechitlán, Balleza 3529 (HUAZ); Valparaíso, Balleza 7497 (HUAZ).

${ }^{*}$ Stevia palmeri A. Gray var. palmeri

HP. Valparaíso, Balleza 7532 (HUAZ).

*Stevia pilosa Lag.

HP. Sombrerete, Balleza 7087 (HUAZ).

${ }^{*}$ Stevia porphyrea McVaugh

HP. Jerez, Balleza 6533 (HUAZ); Miguel Auza, Balleza 6888 (HUAZ); Sombrerete, Balleza 6163 (HUAZ); Teúl de González Ortega, Balleza 2642 (HUAZ); Valparaíso, Balleza 7514 (HUAZ); Zacatecas, Balleza 6964 (HUAZ).

${ }^{*}$ Stevia purpusii B. L. Rob.

HP. Monte Escobedo, Balleza 3887 (HUAZ).

${ }^{*}$ Stevia reticulata Grashoff

HP. Valparaíso, Balleza 9424 (HUAZ).

${ }^{*}$ Stevia rosei B. L. Rob.

HP. Benito Juárez, Balleza 7384 (HUAZ); Monte Escobedo, Balleza 7476 (HUAZ); Valparaíso, Balleza 9440 (HUAZ); Zacatecas, Balleza 6999 (HUAZ).

*Stevia rzedowskii McVaugh

AR. Jalpa, McVaugh 25947 (MEXU); Juchipila, Rzedowski 18262 (ENCB); Tlaltenango de Sánchez Román, Balleza 5247 (HUAZ).

Stevia salicifolia Cav. var. salicifolia

AR. Chalchihuites; Balleza 6106 (HUAZ); Concepción del Oro, Palmer 401 (MEXU); General Joaquín Amaro, Balleza 5947 (HUAZ); Guadalupe, Balleza 7009 (HUAZ); Jalpa, Balleza 6467 (HUAZ); Jerez, Balleza 6510 (HUAZ); Loreto, Balleza 6593 (HUAZ); Nochistlán de Mejía, Balleza 7623 (HUAZ); Pinos, Balleza 5336 (HUAZ); Sombrerete, Fernández 1253 (MEXU); Villanueva, Cronquist 10272 (MEXU); Zacatecas, Balleza 6967 (HUAZ).

${ }^{*}$ Stevia scabrella Benth. var. scabrella

HP. Tlaltenango de Sánchez Román, Balleza 5255 (HUAZ); Valparaíso, McVaugh 25781 (MEXU). 
Anexo. Continuación.

*Stevia serrata Cav. var. arguta B. L. Rob.

HP. Chalchihuites, Balleza 6107 (HUAZ); Genaro Codina, Balleza 10372 (HUAZ); Jerez, Balleza 6517 (HUAZ); Nochistlán de Mejía, Balleza 6496 (HUAZ); Pinos, Balleza 6415 (HUAZ); Sombrerete, Balleza 6164 (HUAZ); Zacatecas, Balleza 6995 (HUAZ).

Stevia serrata Cav. var. serrata

HP. Benito Juárez, Balleza 6332 (HUAZ); Chalchihuites, Balleza 6121 (HUAZ); García de la Cadena, Balleza 7310 (HUAZ); General Joaquín Amaro, Balleza 9511 (HUAZ); Guadalupe, Balleza 7715 (HUAZ); Jalpa, Balleza 8988 (HUAZ); Jerez, Balleza 7021 (HUAZ); Juchipila, Balleza 8907 (HUAZ); Miguel Auza, Balleza 6889 (HUAZ); Monte Escobedo, Balleza 7460 (HUAZ); Nochistlán de Mejía, Balleza 7616 (HUAZ); Sombrerete, García 797 (MEXU); Tlaltenango de Sánchez Román, Balleza 6720 (HUAZ); Valparaíso, Balleza 9299 (HUAZ); Villa Hidalgo, Balleza 10596 (HUAZ); Zacatecas, Balleza 6963 (HUAZ).

Stevia suaveolens Lag.

HP. Nochistlán de Mejía, Balleza 7584 (HUAZ).

*Stevia subpubescens Lag. var. opaca (Sch. Bip.) B. L. Rob.

HP. Jalpa, Balleza 7553 (HUAZ); Juchipila, Balleza 11775 (HUAZ); Nochistlán de Mejía, Balleza 7629 (HUAZ); Teúl de González Ortega, Balleza 8063 (HUAZ).

*Stevia tephra B. L. Rob.

HP. Jerez, Balleza 7059 (HUAZ).

*Stevia tomentosa Kunth

HP. Chalchihuites, Balleza 7151 (HUAZ); Fresnillo, Balleza 7227 (HUAZ); Monte Escobedo, Balleza 3906 (HUAZ); Sombrerete, Balleza 7148 (HUAZ).

*Stevia trifida Lag.

HP. Jalpa, Balleza 8002 (HUAZ); Juchipila, Balleza 7948 (HUAZ); Moyahua de Estrada, Enríquez 1438 (HUAZ).

Stevia viscida Kunth

HP. Benito Juárez, Balleza 7374 (HUAZ); Chalchihuites, Balleza 7168 (HUAZ); García de la Cadena, Balleza 7311 (HUAZ); Jerez, Balleza 7015 (HUAZ); Juchipila, Balleza 8901 (HUAZ); Monte Escobedo, Balleza 7424 (HUAZ); Nochistlán de Mejía, Balleza 7600 (HUAZ); Sombrerete, Balleza 7135 (HUAZ); Tabasco, Balleza 9477 (HUAZ); Teúl de González Ortega, Balleza 7335 (HUAZ); Tlaltenango de Sánchez Román, Rzedowski \& McVaugh 924 (MEXU); Valparaíso, Balleza 9301 (HUAZ); Villanueva, Balleza 3412 (HUAZ).

*Stevia zacatecana McVaugh

AR. Tlaltenango de Sánchez Román, McVaugh 25940 (MEXU).

*Steviopsis adenosperma (Sch. Bip.) B. L. Turner [=Eupatorium adenospermum Sch. Bip. in Seem.] HP. Tlaltenango de Sánchez Román, Rzedowski \& McVaugh 963 (ENCB).

*Steviopsis dryophila (B. L. Rob.) B. L. Turner [=Eupatorium dryophilum B. L. Rob.] HP. Tlaltenango de Sánches Roman, Gentry 18278 (Citado en McVaugh, 1984). 
Acta Botanica Mexicana (2002), 59: 5-69

Anexo. Continuación.

*Steviopsis rapunculoides (DC.) R. M. King \& H. Rob. [=Brickellia rapunculoides (DC.) McVaugh] HP. García de la Cadena, Balleza 7284 (HUAZ).

Steviopsis scoparia (DC.) A. Gray [=Brickellia scoparia (DC.) A. Gray] HP. Jerez, Balleza 7058 (HUAZ); Monte Escobedo, Balleza 7436 (HUAZ); Villanueva, Balleza 7245 (HUAZ).

Steviopsis squamulosa (A. Gray) B. L. Turner

AR. Monte Escobedo, Balleza 5559 (HUAZ); Sombrerete, Balleza 5788 (HUAZ).

*Steviopsis thyrsiflora (A. Gray) B. L. Turner var. solidaginifolia (A. Gray) B. L. Turner [=Brickellia thyrsiflora A. Gray]

HP. Benito Juárez, Balleza 7404a (HUAZ); Jerez, Balleza 7037 (HUAZ); Monte Escobedo, Balleza 7409 (HUAZ); Tabasco, Balleza 9478 (HUAZ).

Steviopsis vigintiseta (DC.) R. M. King \& H. Rob.

HP. Juchipila, Balleza 10077 (HUAZ); Monte Escobedo, Balleza 7495 (HUAZ); Nochistlán de Mejía, Balleza 7605 (HUAZ); Tabasco, Balleza 9464 (HUAZ); Teúl de González Ortega, Balleza 7318 (HUAZ); Tlaltenango de Sánchez Román, Balleza 6728 (HUAZ); Valparaíso, Balleza 9376 (HUAZ).

Tagetes filifolia Lag.

HA. Teúl de González Ortega, Balleza 7326 (HUAZ); Valparaíso, Balleza 9325 (HUAZ).

Tagetes lucida Cav.

HP. Apulco, Balleza 5642 (HUAZ); Benito Juárez, Balleza 6310 (HUAZ); Chalchihuites, Balleza 7167 (HUAZ); Fresnillo, Balleza 6014 (HUAZ); General Francisco Murguía, Balleza 6958 (HUAZ); General Joaquín Amaro, Balleza 9515 (HUAZ); Jalpa, Balleza 6473 (HUAZ); Jerez, Balleza 6526 (HUAZ); Juchipila, Balleza 8369 (HUAZ); Miguel Auza, Balleza 6864 (HUAZ); Monte Escobedo, Balleza 7491 (HUAZ); Nochistlán de Mejía, Balleza 5612 (HUAZ); Sombrerete, Balleza 6192 (HUAZ); Tepechitlán, Balleza 3476 (HUAZ); Teúl de González Ortega, Balleza 6359 (HUAZ); Tlaltenango de Sánchez Román, Balleza 6704 (HUAZ); Valparaíso, Balleza 6046 (HUAZ); Villanueva, Balleza 7251 (HUAZ).

*Tagetes lunulata Ortega

HA. Fresnillo, Balleza 5970 (HUAZ); Genaro Codina, Balleza 10345 (HUAZ); Guadalupe, Balleza 7717 (HUAZ); Jalpa, Balleza 5581 (HUAZ); Jerez, Balleza 6522 (HUAZ); Juan Aldama, Balleza 11179 (HUAZ); Juchipila, Balleza 9046 (HUAZ); Monte Escobedo, Balleza 7448 (HUAZ); Moyahua de Estrada, Balleza 7258b (HUAZ); Nochistlán de Mejía, Balleza 7625 (HUAZ); Noria de Ángeles, Balleza 6600 (HUAZ); Pinos, Balleza 10611 (HUAZ); Sombrerete, Balleza 7105 (HUAZ); Tabasco, Balleza 9459 (HUAZ); Valparaíso, Balleza 6033 (HUAZ); Villa González Ortega, Balleza 7750 (HUAZ); Villanueva, Balleza 3339 (HUAZ); Zacatecas, Balleza 6972 (HUAZ).

Tagetes micrantha Cav.

HA. Benito Juárez, Balleza 7367 (HUAZ); Chalchihuites, Balleza 7164 (HUAZ); García de la Cadena, Balleza 7287 (HUAZ); Genaro Codina, Balleza 10360 (HUAZ); Jalpa, Balleza 8982 (HUAZ); Jerez, Balleza 7016 (HUAZ); Juchipila, Balleza 9036 (HUAZ); Monte Escobedo, Balleza 7413 (HUAZ); Nochistlán de Mejía, Balleza 7598 (HUAZ); Noria de Ángeles, Balleza 10588 (HUAZ); Pinos, Balleza 10603 (HUAZ); Sombrerete, Balleza 7107 (HUAZ); Tabasco, Balleza 9483 (HUAZ); Tlaltenango de Sánchez Román, Balleza 6719 (HUAZ); Valparaíso, Balleza 7521 (HUAZ); Villanueva, Balleza 7247 (HUAZ); Zacatecas, Balleza 6982 (HUAZ). 
Anexo. Continuación.

Tagetes subulata Cerv.

HA. García de la Cadena, Balleza 7307 (HUAZ); Juchipila, Balleza 7860 (HUAZ); Monte Escobedo, García 844 (CHAPA); Nochistlán de Mejía, Balleza 7635b (HUAZ); Valparaíso, Balleza 9368 (HUAZ).

Tagetes tenuifolia Cav. [=Tagetes heterocarpa Rydb.]

AR. Guadalupe, García 294 (MEXU); Tlaltenango de Sánchez Román, Balleza 8524 (HUAZ); Villanueva, Cronquist 10271 (MEXU); Zacatecas, Pringle 1752 (MEXU).

Tagetes triradiata Greenm.

HA. Benito Juárez, Balleza 7365 (HUAZ); García de la Cadena, Balleza 7291 (HUAZ); Juchipila, Balleza 7897 (HUAZ); Nochistlán de Mejía, Balleza 7602 (HUAZ); Teúl de González Ortega, Balleza 7325 (HUAZ); Tlaltenango de Sánchez Román, Rzedowski \& McVaugh 914 (MEXU); Valparaíso, Balleza 9328 (HUAZ).

Taraxacum officinale Wiggers

HP. Concepción del Oro, Balleza 8024 (HUAZ); Guadalupe, Balleza 5272 (HUAZ); Jerez, Balleza 8087 (HUAZ); Loreto, Balleza 8119 (HUAZ); Luis Moya, Balleza 8117 (HUAZ); Mazapil, Balleza 6638 (HUAZ); Miguel Auza, Balleza 8103 (HUAZ); Noria de Ángeles, Balleza 5290 (HUAZ); Ojocaliente, Balleza 8113 (HUAZ); Pinos, Balleza 8942 (HUAZ); Zacatecas, Balleza 136 (HUAZ).

Thelesperma longipes A. Gray

HP. Concepción del Oro, Pinkava s/n (MEXU).

Thelesperma megapotamicum (Spreng.) Kuntze var. megapotamicum

HP. Concepción del Oro, Balleza 6208 (HUAZ); Cuauhtémoc, Balleza 10395 (HUAZ); Fresnillo, Balleza 6007 (HUAZ); Guadalupe, Balleza 10337 (HUAZ); Juan Aldama, Balleza 11311 (HUAZ); Mazapil, Balleza 11128 (HUAZ); Miguel Auza, Balleza 6898 (HUAZ); Villa de Cos, Balleza 10692 (HUAZ); Zacatecas, Balleza 327 (HUAZ).

Thelesperma simplicifolium A. Gray var. simplicifolium HP. Tlaltenango de Sánchez Román, Breedlove 63942 (MEXU).

Thymophylla acerosa (DC.) Strother [=Dyssodia acerosa DC.] HP. Concepción del Oro, Medrano 8139 (MEXU); El Salvador, Balleza 8279 (HUAZ); Fresnillo, Balleza 5676 (HUAZ); General Francisco Murguía, Balleza 5906 (HUAZ); General Pánfilo Natera, Balleza 7728 (HUAZ); Juan Aldama, Balleza 11313 (HUAZ); Loreto, Balleza 5282 (HUAZ); Mazapil, Balleza 6637 (HUAZ); Miguel Auza, Balleza 5850 (HUAZ); Pinos, Balleza 8947 (HUAZ); Río Grande, Balleza 10723 (HUAZ); Villa de Cos, Balleza 7698 (HUAZ); Villa Hidalgo, Balleza 10597 (HUAZ).

Thymophylla pentachaeta (DC.) Small var. hartwegii (A. Gray) Strother [=Dyssodia pentachaeta (DC.) B. L. Rob. var. hartwegii (A. Gray) Strother]

HP. Cuauhtémoc, Balleza 6579 (HUAZ); Jiménez del Teúl, Balleza 7192 (HUAZ); Juan Aldama, Balleza 5838 (HUAZ); Miguel Auza, Balleza 5842 (HUAZ); Pánuco, Balleza 7664 (HUAZ); Sombrerete, Tenorio 2163 (MEXU); Villa de Cos, Balleza 7670 (HUAZ); Villa González Ortega, Balleza 6605 (HUAZ).

Thymophylla pentachaeta (DC.) Small var. pentachaeta [=Dyssodia pentachaeta (DC.) B. L. Rob.] HP. Cañitas de Felipe Pescador, Balleza 11198 (HUAZ); Concepción del Oro, Stanford 489 (MEXU); Cuauhtémoc, Balleza 10414 (HUAZ); El Salvador, Balleza 9732 (HUAZ); Fresnillo, Balleza 5761 
Anexo. Continuación.

(HUAZ); Loreto, Balleza 5283 (HUAZ); Mazapil, Balleza 6616 (HUAZ); Noria de Ángeles, Balleza 6596 (HUAZ); Pinos, Balleza 6439 (HUAZ); Río Grande, Balleza 5694 (HUAZ); Sombrerete, Balleza 7085 (HUAZ); Villa de Cos, Balleza 6799 (HUAZ); Villa González Ortega, Balleza 8124 (HUAZ); Zacatecas, Balleza 365 (HUAZ).

Thymophylla setifolia Lag. [=Dyssodia setifolia (Lag.) B. L. Rob.] HP. Concepción del Oro, Jansen 471 (MEXU); Cuauhtémoc, Balleza 6577 (HUAZ); Fresnillo, Balleza 5753 (HUAZ); General Pánfilo Natera, Balleza 7730 (HUAZ); Loreto, Balleza 5280 (HUAZ); Mazapil, Balleza 9802 (HUAZ); Pánuco, Balleza 7659 (HUAZ); Pinos, Balleza 8241 (HUAZ); Río Grande, Balleza 5693 (HUAZ); Villa de Cos, Balleza 6812 (HUAZ); Villa González Ortega, Balleza 6610 (HUAZ).

*Thymophylla tenuifolia (Cass.) Rydb. [=Dyssodia tenuifolia (Cass.) Loes.]

HA. Concepción del Oro, Strother 465 (MEXU); Río Grande, Johnston 12286 (MEXU); Villanueva, Reed 58241 (MEXU).

Tithonia rotundifolia (Mill.) S. F. Blake

HA. Juchipila, Balleza 9109 (HUAZ); Moyahua de Estrada, Enríquez 1280 (HUAZ).

Tithonia tubiformis (Jacq.) Cass.

HA. Chalchihuites, Balleza 7182 (HUAZ); Cuauhtémoc, Balleza 10412 (HUAZ); El Salvador, Balleza 9739 (HUAZ); Fresnillo, Balleza 5974 (HUAZ); General Joaquín Amaro, Balleza 9519 (HUAZ); Jalpa, Balleza 7542 (HUAZ); Jerez, Balleza 5417 (HUAZ); Juchipila, Balleza 9590 (HUAZ); Mazapil, Balleza 11290 (HUAZ); Miguel Auza, Balleza 6909 (HUAZ); Monte Escobedo, Balleza 7435 (HUAZ); Nochistlán de Mejía, Balleza 5636 (HUAZ); Pánuco, Balleza 7638 (HUAZ); Pinos, Balleza 11322 (HUAZ); Río Grande, Balleza 10767 (HUAZ); Sombrerete, Balleza 7131 (HUAZ); Teúl de González Ortega, Balleza 7342 (HUAZ); Valparaíso, Balleza 6092 (HUAZ); Villa de Cos, Balleza 6861 (HUAZ); Villa González Ortega, Balleza 10577 (HUAZ); Villanueva, Balleza 7244 (HUAZ); Zacatecas, Balleza 3917 (HUAZ).

*Townsendia mexicana A. Gray

HP. Cuauhtémoc, Balleza 10397 (HUAZ); El Salvador, Balleza 9698 (HUAZ); Fresnillo, Balleza 5675 (HUAZ); General Pánfilo Natera, García s/n (CHAPA); Mazapil, Balleza 8320 (HUAZ); Mazapil, Johnston 10452 (MEXU); Ojocaliente, Balleza 4256 (HUAZ); Pánuco, Balleza 7652 (HUAZ); Pinos, Balleza 5301 (HUAZ); Río Grande, Balleza 5682 (HUAZ).

*Tridax balbisioides (Kunth) A. Gray

HA. Genaro Codina, Balleza 10365 (HUAZ); Juan Aldama, Balleza 11165 (HUAZ); Pinos, Balleza 10608 (HUAZ); Sombrerete, Gentry 8472 (MEXU); Valparaíso, Balleza 6021 (HUAZ); Zacatecas, Balleza 6981 (HUAZ).

*Tridax coronopifolia (Kunth) Hemsl.

HA. Huanusco, Hernández 9659 (HUAZ); Noria de Ángeles, Balleza 5292 (HUAZ); Pinos, Balleza 10152 (HUAZ); Río Grande, Balleza 11152 (HUAZ); Tabasco, Balleza 9456 (HUAZ); Villa González Ortega, Balleza 7758 (HUAZ); Villanueva, Cronquist 10269a (MEXU); Zacatecas, Balleza 259 (HUAZ).

${ }^{*}$ Tridax palmeri A. Gray var. palmeri

HA. Pinos, Balleza 10599 (HUAZ). 
Anexo. Continuación.

*Tridax petrophila B. L. Rob. \& Greenm.

HP. Jalpa, Balleza 7548 (HUAZ); Tepechitlán, Balleza 6301 (HUAZ); Teúl de González Ortega, Balleza 7406 (HUAZ); Tlaltenango de Sánchez Román, Balleza 6705 (HUAZ).

Tridax procumbens L.

HP. Moyahua de Estrada; Balleza 7270 (HUAZ).

${ }^{*}$ Trixis angustifolia DC.

AR. Fresnillo, Balleza 5647 (HUAZ); Genaro Codina, Balleza 10363 (HUAZ); General Francisco Murguía, Balleza 5885 (HUAZ); Guadalupe, Balleza 7712 (HUAZ); Jerez, Balleza 5379 (HUAZ); Juan Aldama, Balleza 5829 (HUAZ); Loreto, Balleza 5288 (HUAZ); Mazapil, Balleza 5526 (HUAZ); Miguel Auza, Balleza 5865 (HUAZ); Noria de Ángeles, Balleza 5296 (HUAZ); Pánuco, Balleza 7663 (HUAZ); Pinos, Balleza 5355 (HUAZ); Río Grande, Balleza 10752 (HUAZ); Valparaíso, McVaugh 12023 (MEXU); Villa de Cos, Balleza 6857 (HUAZ); Villa García, Martínez s/n (MEXU); Villa González Ortega, Balleza 10571 (HUAZ); Villanueva, Saunders 1958 (MEXU); Zacatecas, Balleza 360 (HUAZ).

Trixis californica Kellogg var. californica

AR. General Francisco Murguía, Balleza 5905 (HUAZ); Mazapil, Balleza 6671 (HUAZ); Pánuco, Bustos s/n (CHAPA).

*Trixis haenkei Sch. Bip.

AR. Jalpa, Balleza 8007 (HUAZ); Juchipila, Balleza 7959 (HUAZ); Moyahua de Estrada, Balleza 5565 (HUAZ); Nochistlán de Mejía, Balleza 8014b (HUAZ).

${ }^{*}$ Trixis hyposericea S. Watson

AR. Apozol, Enríquez 386 (HUAZ); Jalpa, Balleza 7547 (HUAZ); Juchipila, Balleza 7978 (HUAZ); Tabasco, Balleza 9510 (HUAZ); Villa González Ortega, Balleza 7745 (HUAZ); Villanueva, Saunders 1958 (CHAPA).

*Trixis michuacana Lex. var. longifolia (D. Don) C. E. Anderson

AR. Jalpa, Balleza 8005 (HUAZ); Juchipila, Enríquez 848 (HUAZ); Monte Escobedo, Balleza 5529 (HUAZ); Nochistlán de Mejía, Balleza 5629 (HUAZ); Teúl de González Ortega, Balleza 8058 (HUAZ).

*Varilla mexicana A. Gray var. mexicana

HP. Mazapil, Johnston 11521 (MEXU).

${ }^{*}$ Verbesina angustifolia (Benth.) S. F. Blake

AR. Jerez, Balleza 5411 (HUAZ); Juchipila, Balleza 7862 (HUAZ); Monte Escobedo, García 854 (CHAPA); Nochistlán de Mejía, Balleza 7581 (HUAZ); Tlaltenango de Sánchez Román, Rzedowski \& McVaugh 1027 (MEXU); Valparaíso, Breedlove 58980 (MEXU); Mazapil, Balleza 8795 (HUAZ).

*Verbesina chihuahuensis A. Gray

HP. Concepción del Oro, Balleza 6628 (HUAZ).

Verbesina crocata (Cav.) Less.

AR. Apozol, Balleza 377 (HUAZ); Juchipila, Balleza 7788 (HUAZ); Moyahua de Estrada, Enríquez 1734 (HUAZ); Valparaíso, Balleza 6069 (HUAZ). 
Anexo. Continuación.

Verbesina encelioides (Cav.) Benth. \& Hook. f. subsp. exauriculata (B. L. Rob. \& Greenm.) J. R. Coleman

HA. Concepción del Oro, Balleza 6206 (HUAZ); El Salvador, Balleza 9717 (HUAZ); Fresnillo, Balleza 5996 (HUAZ); General Francisco Murguía, Balleza 6931 (HUAZ); Mazapil, Balleza 6681 (HUAZ); Miguel Auza, Balleza 5841 (HUAZ); Moyahua de Estrada, Balleza 5949 (HUAZ); Pinos, Balleza 6443 (HUAZ); Río Grande, Balleza 5704 (HUAZ); Villa de Cos, Balleza 6779 (HUAZ).

*Verbesina greenmanii Urb.

HP. Teúl de González Ortega, Balleza 7339 (HUAZ).

${ }^{*}$ Verbesina hispida McVaugh

HP. Benito Juárez, Balleza 6348 (HUAZ).

${ }^{*}$ Verbesina hypomalaca B. L. Rob. \& Greenm. var. hypomalaca HP. Jalpa, Balleza 8998 (HUAZ).

*Verbesina longipes Hemsl.

HP. Concepción del Oro, Panero 2193 (MEXU); El Salvador, Balleza 9725; Fresnillo, Balleza 5746 (HUAZ); General Francisco Murguía, Balleza 6947 (HUAZ); Mazapil, Chiang 7912 (MEXU); Pinos, Balleza 8958 (HUAZ); Río Grande, Balleza 10756 (HUAZ); Tlaltenango de Sánchez Román, Stanford 515 (MEXU); Villa de Cos, Balleza 6863 (HUAZ); Villanueva, Panero 2223A (MEXU).

*Verbesina mollis Kunth

HP. Jalpa, Balleza 8999 (HUAZ); Jerez, Balleza 6560 (HUAZ); Juchipila, Balleza 9238 (HUAZ); Tabasco, Balleza 9494 (HUAZ); Tlaltenango de Sánchez Román, Balleza 6729 (HUAZ); Zacatecas, Balleza 6986 (HUAZ).

*Verbesina pantoptera S. F. Blake

HP. Jalpa, Balleza 8977 (HUAZ); Juchipila, Balleza 8732 (HUAZ); Sombrerete, Panero 2194 (MEXU); Tlaltenango de Sánchez Román, Rzedowski \& McVaugh 1001 (MEXU).

${ }^{*}$ Verbesina parviflora (Kunth) S. F. Blake var. parviflora HP. Jalpa, Balleza 6484 (HUAZ). Juchipila, Balleza 8730 (HUAZ); Teúl de González Ortega, Balleza $6397 a$ (HUAZ); Tlaltenango de Sánchez Román, Balleza 8536 (HUAZ).

*Verbesina parviflora (Kunth) S. F. Blake var. zacatecana McVaugh HP. Juchipila, Balleza 8575 (HUAZ); Tlaltenango de Sánchez Román, Ramos 1205CH (MEXU); Valparaíso, Balleza 8422 (HUAZ).

${ }^{*}$ Verbesina pedunculosa (DC.) B. L. Rob.

HP. Jerez, Balleza 6527 (HUAZ); Nochistlán de Mejía, Balleza 6495 (HUAZ); Sombrerete, Balleza 6149 (HUAZ); Teúl de González Ortega, Balleza 6400 (HUAZ); Valparaíso, Balleza 6032 (HUAZ); Villanueva, Panero 2224 (MEXU); Zacatecas, Balleza 517 (HUAZ).

*Verbesina serrata Cav. var. serrata

AR. Genaro Codina, Balleza 6576 (HUAZ); General Francisco Murguía, Balleza 6930 (HUAZ); Jalpa, Balleza 6701 (HUAZ); Jiménez del Teúl, Balleza 7191 (HUAZ); Juchipila, Balleza 7800 (HUAZ); Loreto, Balleza 6595 (HUAZ); Río Grande, Balleza 10708 (HUAZ); Sombrerete, Balleza 7091 (HUAZ); Valparaíso, Balleza 6089 (HUAZ); Villanueva, Balleza 6502 (HUAZ). 
Anexo. Continuación.

*Verbesina virgata Cav. var. virgata

AR. General Joaquín Amaro, Rose 2753 (MEXU); Jalpa, Balleza 6474 (HUAZ); Jerez, Balleza 6563 (HUAZ); Juchipila, Balleza 11750 (HUAZ); Monte Escobedo, Balleza 7455 (HUAZ); Nochistlán de Mejía, Balleza 9032 (HUAZ); Pinos, Balleza 6446 (HUAZ); Tabasco, Balleza 9507 (HUAZ); Tepechitlán, Balleza 3538 (HUAZ); Tlaltenango de Sánchez Román, Balleza 6724 (HUAZ); Valparaíso, Balleza 9310 (HUAZ).

*Vernonanthura liatroides (DC.) H. Rob. [=Vernonia capreifolia Gleason] HP. Juchipila, Balleza 9996 (HUAZ); Moyahua de Estrada, Enríquez 341 (HUAZ).

*Vernonanthura serratuloides (Kunth) H. Rob. [=Vernonia serratuloides Kunth] HP. García de la Cadena, Balleza 7312 (HUAZ); Jalpa, Balleza 7549 (HUAZ); Juchipila, Balleza 7797 (HUAZ); Tepechitlán, Balleza 3516 (HUAZ); Tlaltenango de Sánchez Román, McVaugh 25660 (HUAZ); Valparaíso, Balleza 7498 (HUAZ); Villanueva, García 869 (CHAPA).

*Viguiera brevifolia Greenm.

HP. Concepción del Oro, Rzedowski 9324 (MEXU).

Viguiera cordifolia A. Gray var. cordifolia

HP. Fresnillo, Balleza 5678 (HUAZ); Genaro Codina, Balleza 10371 (HUAZ); Guadalupe, Balleza 7721 (HUAZ); Jerez, Balleza 7070 (HUAZ); Juan Aldama, Balleza 11164 (HUAZ); Juchipila, Balleza 11782 (HUAZ); Mazapil, Balleza 10675 (HUAZ); Pinos, Balleza 10175 (HUAZ); Río Grande, García 1203 (MEXU); Sombrerete, Balleza 6189 (HUAZ); Zacatecas, Balleza 6968 (HUAZ).

Viguiera dentata (Cav.) Spreng.

HP. Apozol, Enríquez 384 (MEXU); Apulco, Balleza 5639 (HUAZ); Concepción del Oro, Balleza 6262 (HUAZ); Fresnillo, Balleza 7226 (HUAZ); Genaro Codina, Balleza 5277 (HUAZ); General Francisco Murguía, Balleza 5902 (HUAZ); Juchipila, Balleza 7794 (HUAZ); Mazapil, Balleza 9743 (HUAZ); Miguel Auza, Balleza 6868 (HUAZ); Monte Escobedo, Balleza 5532 (HUAZ); Moyahua de Estrada, Enríquez 1392 (HUAZ); Nochistlán de Mejía, Balleza 5635 (HUAZ); Noria de Angeles, Balleza 10591 (HUAZ); Pinos, Balleza 11333 (HUAZ); Río Grande, Balleza 10761 (HUAZ); Sombrerete, Tenorio 2186 (MEXU); Tabasco, Balleza 9468 (HUAZ); Tlaltenango de Sánchez Román, Rzedowski \& McVaugh 938 (MEXU); Valparaíso, Balleza 7503 (HUAZ); Villa de Cos, Balleza 10658 (HUAZ); Villa González Ortega, Balleza 10573 (HUAZ); Zacatecas, Balleza 197 (HUAZ).

${ }^{*}$ Viguiera ensifolia (Sch. Bip.) S. F. Blake

HP. Benito Juárez, Balleza 8077 (HUAZ); Juchipila, Balleza 11652 (HUAZ); Teúl de González Ortega, Balleza 8053 (HUAZ); Tlaltenango de Sánchez Román, Balleza 5267 (HUAZ); Valparaíso, Balleza 9393 (HUAZ).

*Viguiera excelsa (Willd.) Benth. \& Hook. f. ex Hemsl. var. pachycephala (DC.) B. L. Turner [=Viguiera pachycephala (DC.) Hemsl.]

HP. García de la Cadena, Balleza 7302 (HUAZ); Jalpa, Balleza 6489 (HUAZ); Juchipila, Balleza 9167 (HUAZ); Monte Escobedo, Balleza 7430 (HUAZ); Moyahua de Estrada, Sundberg 2931 (MEXU); Nochistlán de Mejía, Balleza 6498 (HUAZ); Tabasco, Balleza 9508 (HUAZ); Tepechitlán, Balleza 6300 (HUAZ); Teúl de González Ortega, Balleza 6379 (HUAZ); Tlaltenango de Sánchez Román, Balleza 6721 (HUAZ); Valparaíso, Balleza 9399 (HUAZ). 
Anexo. Continuación.

*Viguiera flava (Hemsl.) S. F. Blake var. flava

HP. Monte Escobedo, Balleza 7486 (HUAZ); Teúl de González Ortega, Balleza 6395 (HUAZ).

*Viguiera hypargyrea Greenm.

HP. Sombrerete, Enríquez 2030 (HUAZ).

${ }^{*}$ Viguiera linearis (Cav.) Sch. Bip. ex Hemsl. var. linearis

HP. Chalchihuites, Balleza 7181 (HUAZ); Cuauhtémoc, Balleza 10411 (HUAZ); Fresnillo, Balleza 5989 (HUAZ); Genaro Codina; Balleza 6569 (HUAZ); General Joaquín Amaro, Balleza 5923 (HUAZ); Jalpa, Balleza 6471 (HUAZ); Jerez, Balleza 6545 (HUAZ); Juan Aldama, Balleza 5819 (HUAZ); Monte Escobedo; Balleza 7410 (HUAZ); Noria de Angeles, Balleza 10593 (HUAZ); Río Grande, Balleza 10739 (HUAZ); Sombrerete, Balleza 7208 (HUAZ); Teúl de González Ortega, Balleza 6399 (HUAZ); Valparaíso, Balleza 7496 (HUAZ); Villa González Ortega, Balleza 10567 (HUAZ); Villanueva, Balleza 5351 (HUAZ); Zacatecas, Balleza 209 (HUAZ).

*Viguiera palmeri A. Gray var. rzedowskii McVaugh

HP. Jalpa, Balleza 7570 (HUAZ); Juchipila, Balleza 10994 (HUAZ); Moyahua de Estrada, Enríquez 1684 (HUAZ); Tabasco, Balleza 9455 (HUAZ).

${ }^{*}$ Viguiera parkinsonii (Hemsl.) S. F. Blake

HP. Juchipila, Balleza 11500 (HUAZ); Tlaltenango de Sánchez Román, Balleza 6769 (HUAZ); Valparaíso, Balleza 9386 (HUAZ).

*Viguiera quinqueradiata (Cav.) A. Gray

A. García de la Cadena, Balleza 7298 (HUAZ); Jalpa, Balleza 7545 (HUAZ); Juchipila, Balleza 7777 (HUAZ); Moyahua de Estrada, Enríquez 231 (MEXU); Nochistlán de Mejía, Balleza 7635a (HUAZ); Tabasco, Balleza 9535 (HUAZ); Valparaíso, Balleza 7501 (HUAZ); Villanueva, Cronquist 10274 (MEXU).

*Viguiera rosei Greenm.

HP. Monte Escobedo, Balleza 4160 (HUAZ); Villanueva, Balleza 7232 (HUAZ).

*Viguiera schultzii S. F. Blake

HP. Teúl de González Ortega, Balleza 7351 (HUAZ).

${ }^{*}$ Viguiera sessilifolia DC.

HP. Benito Juárez, Balleza 7389 (HUAZ); Monte Escobedo, Balleza 7452 (HUAZ); Nochistlán de Mejía, Balleza 7599 (HUAZ); Tabasco, Balleza 9497 (HUAZ); Tlaltenango de Sánchez Román, Rzedowski \& McVaugh 995 (MEXU).

Viguiera stenoloba S. F. Blake

HP. Concepción del Oro, Gonzáles 9000 (MEXU); Mazapil, Balleza 5461 (HUAZ); Villa de Cos, Balleza 10659 (HUAZ).

${ }^{*}$ Wedelia grayi McVaugh

HP. Valparaíso, Balleza 8416 (HUAZ).

*Wedelia rosei (Greenm.) McVaugh

AR. Tlaltenango de Sánchez Román, Rzedowski \& McVaugh 1002 (MEXU). 
Anexo. Continuación.

Xanthium strumarium L.

HA. Apozol, Faudón s/n (MEXU); Cuauhtémoc, Balleza 10409 (HUAZ); El Salvador, Balleza 9734 (HUAZ); Fresnillo, Balleza 5965 (HUAZ); General Francisco Murguía, Balleza 6954 (HUAZ); Guadalupe, Balleza 10340 (HUAZ); Juchipila, Faudón s/n (CHAPA); Mazapil, Balleza 11067 (HUAZ); Miguel Auza, Balleza 6908 (HUAZ); Moyahua de Estrada, Balleza 7258 (HUAZ); Río Grande; Balleza 10736 (HUAZ); Tepechitlán, Balleza 3537 (HUAZ); Valparaíso, Balleza 6080 (HUAZ); Villa de Cos, Balleza 10325 (HUAZ); Villa González Ortega, Balleza 10576 (HUAZ); Zacatecas, Balleza 662 (HUAZ).

*Xanthocephalum benthamianum Hemsl.

HA. General Joaquín Amaro, Rose 2720 (MEXU); Sombrerete, Sundberg 2909 (MEXU).

*Xanthocephalum gymnospermoides (A. Gray) Benth. var. eglandulosum McVaugh

HA. Benito Juárez, Balleza 7393 (HUAZ); Chalchihuites, Balleza 7176 (HUAZ); General Joaquín Amaro, Balleza 9530 (HUAZ); Jerez, Balleza 6557 (HUAZ); Loreto, Balleza 6585 (HUAZ); Miguel Auza, Balleza 6893 (HUAZ); Monte Escobedo, Balleza 7489 (HUAZ); Sombrerete; Balleza 7089 (HUAZ); Sombrerete, Balleza 7224 (HUAZ); Teúl de González Ortega, Balleza 7348 (HUAZ); Villanueva; Balleza 7241 (HUAZ).

*Xylothamia parrasana (S. F. Blake) G. L. Nesom

HP. Mazapil, Johnston 11542 (CHAPA).

*Zaluzania augusta (Lag.) Sch. Bip. var. augusta

AR. Zacatecas, Hernández 9428 (MEXU).

*Zaluzania augusta (Lag.) Sch. Bip. var. rzedowskii McVaugh

AR. Jiménez del Teúl, Balleza 7190 (HUAZ); Valparaíso, Balleza 9448 (HUAZ); Villa González Ortega, Balleza 7756 (HUAZ).

*Zaluzania delgadoana B. L. Turner

AR. Moyahua de Estrada, Saunders 1972 (CHAPA).

*Zaluzania mollissima A. Gray

AR. Chalchihuites, Balleza 6114 (HUAZ); Concepción del Oro, Pennell 17415 (MEXU); General Pánfilo Natera, Balleza 7731 (HUAZ); Mazapil, Balleza 6614 (HUAZ); Villa González Ortega, Balleza 6609 (HUAZ).

*Zaluzania triloba (Ortega) Pers.

HP. Concepción del Oro, Balleza 6255 (HUAZ); El Salvador, Balleza 9701 (HUAZ); Fresnillo, Villarreal 2287 (CHAPA); General Pánfilo Natera, Balleza 7726 (HUAZ); Guadalupe, Balleza 7716 (HUAZ); Juan Aldama, Balleza 11307 (HUAZ); Mazapil, Balleza 6277 (HUAZ); Pánuco, Balleza 7637 (HUAZ); Pinos, Balleza 6408 (HUAZ); Río Grande, Balleza 10753 (HUAZ); Villa de Cos, Balleza 6797 (HUAZ); Villa González Ortega, Balleza 7748 (HUAZ).

Zinnia acerosa (DC.) A. Gray

HP. Cañitas de Felipe Pescador, Balleza 11200 (HUAZ); Concepción del Oro, Medrano 7972 (MEXU); El Salvador, Balleza 8280 (HUAZ); Fresnillo, Balleza 5673 (HUAZ); General Francisco Murguía, Balleza 5907 (HUAZ); General Pánfilo Natera, Balleza 7732 (HUAZ); Juan Aldama, Balleza 
Anexo. Continuación.

5836 (HUAZ); Loreto, Balleza 6583 (HUAZ); Mazapil, Balleza 5475 (HUAZ); Miguel Auza, Balleza 5849 (HUAZ); Noria de Ángeles, Balleza 6599 (HUAZ); Pánuco, Balleza 7662 (HUAZ); Pinos, Balleza 8940 (HUAZ); Río Grande, Balleza 5684 (HUAZ); Sombrerete, Balleza 6187 (HUAZ); Villa de Cos, Balleza 6780 (HUAZ); Villa de Cos, Balleza 6804 (HUAZ); Villa González Ortega, Balleza 6604 (HUAZ).

Zinnia americana (Mill.) Olorode \& A. M. Torres

HA. Juchipila, Balleza 8856 (HUAZ); Moyahua de Estrada, Enríquez 1133 (HUAZ).

*Zinnia angustifolia Kunth var. angustifolia

HP. Benito Juárez, Balleza 6320 (HUAZ); García de la Cadena, Balleza 7296 (HUAZ); Juchipila, Balleza 7837 (HUAZ); Monte Escobedo, Balleza 5560 (HUAZ); Moyahua de Estrada, Balleza 7266b (HUAZ); Tabasco, Balleza 9484 (HUAZ); Tepechitlán, Balleza 3503 (HUAZ); Teúl de González Ortega, Balleza 6355 (HUAZ); Tlaltenango de Sánchez Román, Balleza 6725 (HUAZ); Valparaíso, Balleza 9398 (HUAZ).

*Zinnia angustifolia Kunth var. greggii (B. L. Rob. \& Greenm.) McVaugh

HP. Benito Juárez, Balleza 6341 (HUAZ); Mazapil, Balleza 4085 (HUAZ); Monte Escobedo, Balleza 7463 (HUAZ); Tlaltenango de Sánchez Román, Balleza 6703 (HUAZ).

*Zinnia bicolor (DC.) Hemsl.

HA. Juchipila, Balleza 8917 (HUAZ); Moyahua de Estrada, Enríquez 1333 (HUAZ).

*Zinnia haageana Regel

HA. Juchipila, Balleza 9671 (HUAZ); Teúl de González Ortega, Balleza 6396 (HUAZ).

*Zinnia juniperifolia (DC.) A. Gray

HP. Concepción del Oro, Balleza 6216 (HUAZ); Mazapil, Balleza 6286 (HUAZ).

Zinnia peruviana (L.) L.

HA. Apozol; Enríquez 589 (HUAZ); Genaro Codina, Balleza 10358 (HUAZ); General Francisco Murguía, Balleza 6927 (HUAZ); Jalpa, Balleza 6461 (HUAZ); Juan Aldama, Balleza 11177 (HUAZ); Juchipila, Balleza 7913 (HUAZ); Miguel Auza, Balleza 6871 (HUAZ), Moyahua de Estrada, Enríquez 1087 (HUAZ); Nochistlán de Mejía, Balleza 9017 (HUAZ); Noria de Ángeles, Balleza 7771 (HUAZ); Pánuco, Bustos s/n (CHAPA); Río Grande, Balleza 10732 (HUAZ); Sombrerete, Tenorio 2176 (MEXU); Tabasco, Balleza 9461 (HUAZ); Teúl de González Ortega, Balleza 6402 (HUAZ); Valparaíso, Balleza 7499 (HUAZ); Villanueva, Balleza 3363 (HUAZ). 
Anexo. Continuación.

Especies no registradas en Zacatecas, pero cuya existencia en el estado es muy probable dada su presencia en estados vecinos.

Brickellia cavanillesii (Cass.) A. Gray

AR. Registrada de Durango, Jalisco, San Luis Potosí.

*Brickellia oreithales (B. L. Rob.) Shinners

HP. Registrada de Aguascalientes, Durango, Jalisco.

*Brickellia secundiflora (Lag.) A. Gray var. parryi

A. Registrada de Coahuila, Jalisco y San Luis Potosí.

${ }^{*}$ Chaptalia hololeuca Greene

HP. Registrada de Aguascalientes, Coahuila y San Luis Potosí.

Cosmos diversifolius Otto ex Knowles \& Westc. var. diversifolius

HP. Registrada de Durango, Jalisco y San Luis Potosí.

Heliomeris longifolia (B. L. Rob. \& Greenm.) Cockerell var. longifolia [=Viguiera longifolia (B. L. Rob. \& Greenm.) S. F. Blake]

HP. Registrada de Durango, Jalisco y San Luis Potosí.

Heliomeris multiflora Nutt. var. multiflora

HP. Registrada de Coahuila, Durango, Jalisco y San Luis Potosí.

Hieracium crepidispermum Fries

HP. Registrada de Coahuila, Durango, Jalisco y San Luis Potosí.

*Leucactinia bracteata (S. Watson) Rydb. [=Dyssodia bracteata (S. Watson) S. F. Blake] HP. Registrada de Aguascalientes, Coahuila y San Luis Potosí.

*Steviopsis thyrsiflora (A. Gray) B. L. Turner var. thyrsiflora [=Brickellia thyrsiflora A. Gray] HP. Registrada de Aguascalientes, Coahuila, Durango, Jalisco y San Luis Potosí.

Tagetes erecta L. [=Tagetes heterocarpa Rydb.]

HA. Registrada de Aguascalientes, Coahuila, Jalisco y San Luis Potosí.

${ }^{*}$ Viguiera greggii (A. Gray) S. F. Blake

HP. Registrada de Coahuila, Durango y San Luis Potosí. 